\title{
The prism manifold realization problem
}

\author{
WiLLIAM BALLINGER \\ Chloe Ching-Yun Hsu \\ WYATT MACKEY \\ Yi Ni \\ TYNAN OCHSE \\ FARAMARZ VAFAEE
}

\begin{abstract}
The spherical manifold realization problem asks which spherical three-manifolds arise from surgeries on knots in $S^{3}$. In recent years, the realization problem for C-, T-, O- and I-type spherical manifolds has been solved, leaving the D-type manifolds (also known as the prism manifolds) as the only remaining case. Every prism manifold can be parametrized as $P(p, q)$ for a pair of relatively prime integers $p>1$ and $q$. We determine a list of prism manifolds $P(p, q)$ that can possibly be realized by positive integral surgeries on knots in $S^{3}$ when $q<0$. Based on the forthcoming work of Berge and Kang, we are confident that this list is complete. The methodology undertaken to obtain the classification is similar to that of Greene for lens spaces.
\end{abstract}

57M25, 57R65

\section{Introduction}

There are many notions of simplicity for closed three-manifolds. Perhaps the simplest is that of a manifold with a finite fundamental group. One of the most prominent problems in three-manifold topology is to indicate the list of the simplest closed three-manifolds that can be realized by the simplest three-dimensional topological operations. Given that every closed three-manifold can be obtained by performing surgery on a link in $S^{3}$ (see Lickorish [14] and Wallace [23]), the aforementioned realization problem may be stated as follows:

Question 1.1 Which closed 3-manifolds with finite fundamental groups can be realized by surgeries on nontrivial knots in $S^{3}$ ? 
By the work of Thurston [22], any knot in $S^{3}$ is precisely one of a torus knot, a satellite knot or a hyperbolic knot. Moser [15] classified all finite surgeries on torus knots. Later, Boyer and Zhang [4, Corollary 1.4] showed that if surgery on a satellite knot $K \subset S^{3}$ results in a manifold with a finite fundamental group, then $K$ must be a cable of a torus knot. Such surgeries are classified by Bleiler and Hodgson in [3, Theorem 7]. In regard to the surgery coefficient, Culler-Gordon-Luecke-Shalen [5] proved that any cyclic surgery must be integral. As further proved by Boyer and Zhang [4, Theorem 1.1], the coefficient of any finite surgery is either $p$ or $p / 2$ for some integer $p$. Li and $\mathrm{Ni}$ [13] showed that if half-integral surgery on a hyperbolic knot results in a manifold $Y$ with a finite fundamental group, then $Y$ is homeomorphic to $p / 2$ surgery on either a torus knot or a cable of a torus knot. As a result, we henceforth restrict attention to integral surgeries.

Using Perelman's geometrization theorem, closed three-manifolds with finite fundamental groups can be characterized as those three-manifolds that admit spherical geometry. A spherical 3-manifold (also known as an elliptic 3-manifold) has the form

$$
Y=S^{3} / G
$$

where $G$ is a finite subgroup of $\mathrm{SO}(4)$ that acts freely on $S^{3}$ by rotations. The center $Z=Z(G)$ of $G \cong \pi_{1}(Y)$ is necessarily a cyclic group. According to the structure of $G / Z$, spherical manifolds (besides $S^{3}$ ) are divided into five types: C or cyclic, D or dihedral, $\mathrm{T}$ or tetrahedral, $\mathrm{O}$ or octahedral, I or icosahedral. In particular, if $G / Z$ is the dihedral group

$$
D_{2 p}=\left\langle x, y \mid x^{2}=y^{2}=(x y)^{p}=1\right\rangle
$$

for some integer $p>1$, we get the D-type manifolds. These manifolds are also known as the prism manifolds.

Greene [10] solved the integer surgery realization problem (that is, Question 1.1 when the surgery coefficient is integral) for lens spaces, namely, the C-type manifolds. Later, $\mathrm{Gu}$ [12] provided the solution for T-, O- and I-type manifolds. This leaves the D-type manifolds as the only remaining case, and that is the theme of the present work.

There are many more prism manifolds than any other type of spherical manifolds. It is straightforward to check that for each integer $m>0$, there are only finitely many spherical manifolds $Y$ of other types with $\left|H_{1}(Y)\right|=m$. However, for each $m$ divisible by 4 , there are infinitely many prism manifolds with the order of the first singular homology equal to $m$. To justify, let $P(p, q)$ be the oriented prism manifold with 
Seifert invariants

$$
(-1 ;(2,1),(2,1),(p, q))
$$

where $p>1$ and $q$ are a pair of relatively prime integers. These manifolds satisfy

$$
\left|H_{1}(P(p, q))\right|=4|q| \text {. }
$$

Therefore, any integer $p>1$ relatively prime to $q$ will give a prism manifold $P(p, q)$ with the desired order of the first singular homology. In regard to the realization problem, however, we still have a finiteness result. It was first proved by Doig in [7] that, for a fixed $|q|$, there are only finitely many $p$ for which $P(p, q)$ may be realized by surgery on a knot $K \subset S^{3}$. Later, Ni and Zhang [16] proved an explicit bound for $p$ in terms of $q$ :

$$
p<4|q|
$$

We are now in a position to state the main result of the paper:

Theorem 1.2 Given a pair of relatively prime integers $p>1$ and $q<0$, if the prism manifold $P(p, q)$ can be obtained by $4|q|$-Dehn surgery on a knot $K$ in $S^{3}$, then $P(p, q)$ belongs to one of the six families in Table 1. Moreover, the knot Floer homology group of $K$ is determined by $P(p, q)$ and the family containing it.

Remark 1.3 The six families in Table 1 are divided so that each changemaker vector (see Definition 1.5) corresponds to a unique family. However, a prism manifold $P(p, q)$ could belong to different families, and not just a unique one. In particular, given $P(p, q)$, there may be several knots with surgery yielding $P(p, q)$, and such that these knots have different knot Floer homology groups. We will address the overlaps between these families in Section 13.1. See Table 3.

All the known examples of integral cyclic surgeries (lens space surgeries) come from Berge's primitive/primitive (or simply $\mathrm{P} / \mathrm{P}$ ) construction [1]. There is a generalization of this construction to Seifert-fibered surgeries due to Dean [6], called the primitive/Seifertfibered (or P/SF) construction. See Definition 13.3. The surface slope Dehn surgery on a hyperbolic P/SF knot results in a Seifert-fibered space. Berge and Kang [2] classified all P/SF knots in $S^{3}$. Further, they specified the indices of the singular fibers of the Seifert-fibered manifolds resultant from the surface slope surgeries on such knots. Since prism manifolds are Seifert-fibered spaces over $S^{2}$ with three singular fibers of indices $(2,2, p)$, following from the work of Berge and Kang, we obtain a list of prism 
manifolds that are realizable by knot surgeries. It turns out that this list coincides with Table 1 when $q<0$. However, since Berge and Kang's work is not publicly available, we will not claim that we have resolved the realization problem for prism manifolds when $q<0$. Instead, we will state this part of the realization problem as a conjecture.

Conjecture 1.4 For any $P(p, q)$ in Tables 1 and 2, there exists a knot $K$ such that the $4|q|$-Dehn surgery on $K$ results in $P(p, q)$.

Our study (not included in this paper) of the Berge-Kang classification indicates that the knot $K$ in Conjecture 1.4 can be taken to be a Berge-Kang knot.

The methodology undertaken to prove Theorem 1.2 is inspired from that of Greene [10]. A prism manifold $P(p, q)$ with $q<0$ naturally bounds a negative definite four-manifold $X(p, q)$. See Section 2 . Suppose that $P(p, q)$ is realized by $4|q|$-surgery on a knot $K \subset S^{3}$. In particular, $P(p, q)$ bounds the two-handle cobordism $W_{4|q|}=W_{4|q|}(K)$, obtained by attaching a two-handle to $D^{4}$ along $K \subset S^{3}$ with framing $4|q|$. Note that the surgery coefficient is dictated by homology considerations: see equation (2). The four-manifold $Z:=X(p, q) \cup-W_{4|q|}$ is a smooth, closed, negative definite 4-manifold with $b_{2}(Z)=n+4$, where $n+3=b_{2}(X(p, q))$ for some $n \geq 1$. Donaldson's Theorem A [8] implies that the intersection pairing on $H_{2}(Z)$ is isomorphic to $-\mathbb{Z}^{n+4}$, the negative of the standard $(n+4)$-dimensional Euclidean integer lattice. Consequently, the negative of the intersection pairing on $X(p, q)$, denoted $\Delta(p, q)$, embeds as a codimension-one sublattice of $\mathbb{Z}^{n+4}$. For the prism manifold $P(p, q)$ to arise from a knot surgery, this already gives a restriction on the pair $(p, q)$. We then appeal to the innovative work of Greene that provides even more constraints on $\Delta(p, q)$. To state this essential step, we first need to make a combinatorial definition.

Definition 1.5 A vector $\sigma=\left(\sigma_{0}, \sigma_{1}, \ldots, \sigma_{n+3}\right) \in \mathbb{Z}^{n+4}$ that satisfies $0 \leq \sigma_{0} \leq \sigma_{1} \leq$ $\cdots \leq \sigma_{n+3}$ is a changemaker vector if for every $k$ with $0 \leq k \leq \sigma_{0}+\sigma_{1}+\cdots+\sigma_{n+3}$, there exists a subset $S \subset\{0,1, \ldots, n+3\}$ such that $k=\sum_{i \in S} \sigma_{i}$.

The lattice embedding theorem of Greene [11, Theorem 3.3] now reads as follows: if $P(p, q)$ with $q<0$ is realized by $4|q|$-surgery on $K \subset S^{3}$, then $\Delta(p, q)$ embeds into $\mathbb{Z}^{n+4}$ as the orthogonal complement of a changemaker vector $\sigma \in \mathbb{Z}^{n+4}$. By determining the pairs $(p, q)$ which pass this refined embedding restriction, we get the list of all prism manifolds that could possibly be realized by positive integral surgeries on knots. 
Theorem 1.6 Given a pair of relatively prime integers $p>1$ and $q<0$, we have that $\Delta(p, q) \cong(\sigma)^{\perp}$ for a changemaker vector $\sigma \in \mathbb{Z}^{n+4}$ if and only if $P(p, q)$ belongs to one of the six families in Theorem 1.2. If we further assume that Conjecture 1.4 holds true in this case, then there exists a knot $K \subset S^{3}$ and an isomorphism of lattices

$$
\phi:\left(\mathbb{Z}^{n+4}, I\right) \rightarrow\left(H_{2}(Z),-Q_{Z}\right)
$$

satisfying the property that $\phi(\sigma)$ is a generator of $H_{2}\left(-W_{4|q|}\right)$. Here $I$ is the standard inner product on $\mathbb{Z}^{n+4}$ and $Q_{Z}$ is the intersection form of $Z=X(p, q) \cup-W_{4|q|}$.

\subsection{Prism manifolds $P(p, q)$ with $q>0$}

As discussed, digging up the list of P/SF knots in $S^{3}$ given in [2], we obtain a family of knots with prism manifold surgeries. We can get a list $\mathcal{P}^{+}$of prism manifolds with $q>0$ arising from surgeries on knots in $S^{3}$. See Table 2. In light of Theorem 1.2, we make the following conjecture.

Conjecture 1.7 Given a pair of relatively prime integers $p>1$ and $q>0$, if $P(p, q)$ can be obtained by $4 q-$ Dehn surgery on a knot $K$ in $S^{3}$, then $P(p, q) \in \mathcal{P}^{+}$.

Theorem 1.2 leaves open the integer surgery realization problem for manifolds $P(p, q)$ with $q>0$, and Conjecture 1.7 proposes the solution. A natural direction to pursue is to indicate the list of all knots in $S^{3}$ that admit surgeries to spherical manifolds. In [1], Berge proposed a complete list of knots in $S^{3}$ with lens space surgeries. Indeed, Berge's conjecture states that the P/P knots form a complete list of knots in $S^{3}$ that admit lens space surgeries. All the known examples of spherical manifolds arising from knot surgeries will provide supporting evidence to the following conjecture:

Conjecture 1.8 Let $K$ be a knot in $S^{3}$ that admits a surgery to a spherical manifold. Then $K$ is either a $P / S F$ or a P/P knot.

We point out that Conjecture 1.8 implies Conjecture 1.7.

When $q>p, P(p, q)$ is the double branched cover of $S^{3}$ with branching locus being an alternating Montesinos link, thus it is the boundary of a sharp 4-manifold; see Ozsváth and Szabó [20]. Greene's strategy can still be used to study the realization problem in this case, but the lattices will not be of D-type. We plan to address this case in a future paper. 


\subsection{Organization}

In Section 2, we give the basic topological properties of prism manifolds, and explain how to reduce the realization problem to a problem about lattices. In Section 3, we study the D-type lattices which are central in our paper. There is a natural vertex basis for a D-type lattice. Every vector in the vertex basis is irreducible. A classification of irreducible vectors is given in Proposition 3.9. In Section 4, we endow a changemaker lattice $(\sigma)^{\perp}$ with a standard basis $S$, and study the question when such a lattice is isomorphic to a D-type lattice. From the standard basis elements of a changemaker lattice we can form an intersection graph; see Definition 4.16. We collect many structural results about this graph.

Section 5 addresses some technical lemmas regarding the nonexistence of certain edges in the intersection graph associated to a changemaker lattice. The elements of a standard basis $S$, viewed as an ordered set, are of three types: tight, just right, and gappy (Definition 4.1). As it turns out, the classification of changemaker lattices that are isomorphic to D-type lattices relies highly on the properties of one specific element $v_{f-1}$ of the standard basis: whether it is tight, just right or gappy, together with its placement in $S$. Accordingly, we will do a case-by-case analysis to enumerate the possible standard bases for such a lattice. This occupies Sections 6-11. Section 12 is devoted to converting these standard bases into vertex bases to get a list of pairs $(p, q)$ corresponding to the D-type lattices found in Sections 6-11.

In Section 13 we discuss how one may tabulate all the Berge-Kang P/SF knots that admit prism manifold surgeries. Thus we finish the proofs of Theorems 1.2 and 1.6.

Acknowledgements Ni was partially supported by NSF grant number DMS-1252992 and an Alfred P Sloan Research Fellowship. Ballinger, Hsu, Mackey and Ochse were supported by Caltech's Summer Undergraduate Research Fellowships (SURF) program. Ballinger also wishes to thank Samuel P and Frances Krown for their generous support through the SURF program. We are grateful to John Berge for sending us the preprint [2] and some useful programs. We thank Zhengyuan Shang for finding a typo in Table 3. We thank the referee for a very thorough review.

\section{Background}

In this section, we start with recalling some basic facts about prism manifolds, then provide a concise strategy to translate the prism manifold realization problem into a 


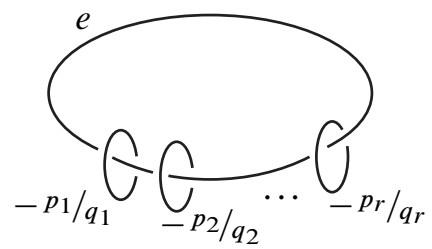

Figure 1: The surgery diagram of a Seifert-fibered space.

lattice theory question. Meanwhile, the necessary background from Heegaard Floer homology will be cited.

\subsection{Prism manifolds}

It is well known that every spherical manifold is a Seifert-fibered space, that is, a three-manifold with a surgery diagram as depicted in Figure $1 .^{1}$ The data

$$
\left(e ;\left(p_{1}, q_{1}\right),\left(p_{2}, q_{2}\right), \ldots,\left(p_{r}, q_{r}\right)\right)
$$

are called the Seifert invariants, where $e$ is an integer, and $\left(p_{1}, q_{1}\right), \ldots,\left(p_{r}, q_{r}\right)$ are pairs of relatively prime integers such that $p_{i}>1$. The rational number

$$
e_{\mathrm{orb}}:=e+\sum_{i=1}^{r} \frac{q_{i}}{p_{i}}
$$

is called the orbifold Euler number. The oriented homeomorphism type of a Seifertfibered space is determined by the multiset

$$
\left\{\frac{q_{i}}{p_{i}}-\left\lfloor\frac{q_{i}}{p_{i}}\right\rfloor \mid i=1, \ldots, r\right\}
$$

together with $e_{\mathrm{orb}}$. It is elementary to verify that if a Seifert-fibered space is a rational homology sphere, it must be the case that $e_{\text {orb }} \neq 0$, and

$$
\left|H_{1}(Y)\right|=p_{1} p_{2} \cdots p_{r}\left|e_{\text {orb }}\right| .
$$

For a pair of relatively prime integers $p>1$ and $q$, a prism manifold $P(p, q)$ is a Seifert-fibered space over $S^{2}$ with three singular fibers of indices $(2,2, p)$, having the Seifert invariants

$$
(-1 ;(2,1),(2,1),(p, q)) .
$$

It is well known that $P(p, q)$ has exactly two Seifert fibrations, and the above one is the only fibration over an orientable base orbifold [21]. As a result, the orbifold

\footnotetext{
${ }^{1}$ We only consider Seifert-fibered spaces whose base orbifold has genus zero.
} 
Euler number of the above Seifert fibration, which is $q / p$, is a topological invariant for $P(p, q)$. Hence $P\left(p_{1}, q_{1}\right) \cong P\left(p_{2}, q_{2}\right)$ if and only if $\left(p_{1}, q_{1}\right)=\left(p_{2}, q_{2}\right)$. Here “ $\cong$ " denotes orientation-preserving homeomorphism.

Following from their Seifert-fibered presentations, prism manifolds enjoy the symmetry

$$
P(p,-q) \cong-P(p, q),
$$

where $-P(p, q)$ is the manifold $P(p, q)$ with opposite orientation. The fundamental group of $P(p, q)$ has presentation

$$
\pi_{1}(P(p, q))=\left\langle x, y \mid x y x^{-1}=y^{-1}, x^{2|q|}=y^{p}\right\rangle .
$$

The center of this group is a cyclic group of order $2|q|$ generated by $x^{2}$. It follows from (5) that $H_{1}(P(p, q))$ is cyclic if and only if $p$ is odd. Hence if $P(p, q)$ is obtained by surgery on a knot in $S^{3}, p$ must be odd.

Lemma 2.1 Suppose that $P(p, q)$ is obtained by $4|q|-$ surgery on a knot $K \subset S^{3}$. If $K$ is a torus knot, then $(p, q)=(2 k+1, k)$ or $(2 k+1,-k-1)$ for some $k>0$, and $K$ is $T(2 k+1,2)$. If $K$ is a satellite knot, then either $(p, q)=(2 k+1,9 k+4)$ for some $k>0$, and $K$ is the $(12 k+5,3)-$ cable of $T(2 k+1,2)$, or $(p, q)=(2 k+1,-9 k-5)$ for some $k>0$, and $K$ is the $(12 k+7,3)$-cable of $T(2 k+1,2)$.

Proof First suppose that $P(p, q)$ is obtained by $4|q|$-surgery on a torus knot $T(r, s)$. On one hand, it follows from [15, Proposition 3.1] that if $4|q|$ Dehn surgery along $T(r, s)$ results in a Seifert-fibered manifold with three singular fibers, then the fibers have indices $r, s$ and $|r s-4| q||$. On the other hand, the prism manifold $P(p, q)$ has fibers of indices 2, 2, $p$. By comparing the indices we find that the torus knot is $T(2 k+1,2)$. This implies that $p=2 k+1$, and we can deduce that $|q|=k$ or $k+1$. Note that the slope of the Seifert fiber of the complement of $K$ is $4 k+2$. If $|q|=k$, since $0<4 k<4 k+2$, the orbifold Euler number of the resulting manifold is positive; that is, $q=k$. If $|q|=k+1$, since $0<4 k+2<4 k+4$, the orbifold Euler number of the resulting manifold is negative; that is, $q=-k-1$.

When $K$ is a satellite knot, we deduce from [4, Corollary 1.4] that $K$ must be a cable of a torus knot. Therefore, we can use the classification of finite surgeries on such knots in [3, Theorem 7]: the classification is given in [3, Table 1]. It follows that $K$ is the $(12 k+6 \mp 1,3)$-cable of $T(2 k+1,2)$, and $|q|=9 k+4$ or $9 k+5$. When $|q|=9 k+4$, the $(36 k+16)$-surgery on the $(12 k+5,3)$-cable of $T(2 k+1,2)$ is the 


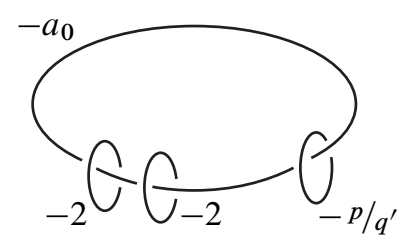

Figure 2: A surgery diagram of $P(p, q)$.

same as the $\frac{1}{9}(36 k+16)-$ surgery on $T(2 k+1,2)$. Since the resulting manifold has a singular fiber of index $2 k+1, p=2 k+1$. Similarly, we can deal with the case $|q|=9 k+5$.

Remark 2.2 In [16], it is proved that if $P(2 k+1, k)$ or $P(2 k+1,-k-1)$ can be obtained by positive surgery on a knot $K \subset S^{3}$, then $K$ must be $T(2 k+1,2)$.

One key step in the proof of Theorem 1.2 is that every prism manifold $P(p, q)$ with $q<0$ bounds a negative definite four-manifold. Writing

$$
k=\left\lfloor\frac{q}{p}\right\rfloor<0, \quad q^{\prime}=q-k p>0,
$$

then $P(p, q)$ has an equivalent Seifert fibration with Seifert invariants

$$
\left(-1+k ;(2,1),(2,1),\left(p, q^{\prime}\right)\right) .
$$

Correspondingly, we get a surgery diagram for $P(p, q)$ as in Figure 2, where $a_{0}=$ $1-k \geq 2$. Having $p / q^{\prime}>1$, expand $p / q^{\prime}$ in a continued fraction

$$
\frac{p}{q^{\prime}}=\left[a_{1}, \ldots, a_{n}\right]^{-}:=a_{1}-\frac{1}{a_{2}-\frac{1}{a_{3}-\frac{1}{\ddots \cdot-\frac{1}{a_{n}}}}},
$$

where the $a_{i}$ are integers satisfying $a_{i} \geq 2$. Equivalently, we can write

$$
\frac{-q}{p}=\left[a_{0}-1, a_{1}, \ldots, a_{n}\right]^{-} \text {. }
$$

Let $X(p, q)$ be the four-manifold that $P(p, q)$ bounds, obtained by attaching twohandles to $D^{4}$ instructed by the framed link in Figure 3. More precisely, each unknot component in Figure 3 denotes a disk bundle over $S^{2}$ with Euler number specified by its coefficient. The manifold $X(p, q)$ is obtained from plumbing these disk bundles together: two disk bundles are plumbed if the corresponding unknot components are linked. 


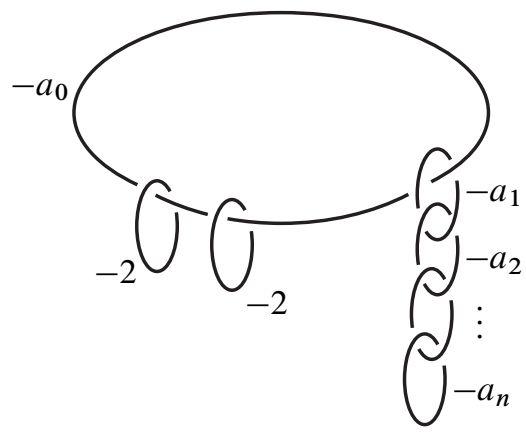

Figure 3: An integral surgery diagram of $P(p, q)$.

Let

$$
Q_{X}: H_{2}(X) \times H_{2}(X) \rightarrow \mathbb{Z}
$$

denote the intersection pairing on $X=X(p, q)$. The second homology of $X$ has rank $n+3$, generated by elements $x_{*}, x_{* *}, x_{0}, \ldots, x_{n}$. Note that $x_{*}$ and $x_{* *}$ correspond to the vertices with weights -2 in Figure 3.

Lemma 2.3 $X(p, q)$ is a negative definite four-manifold.

Proof We will show that $-Q_{X}$ is positive definite. Given a vector $v \in H_{2}(X)$, for each $i=1, \ldots, n$, it is easy to check that $-Q_{X}(v, v)$ is an increasing function of the $a_{i}$. In particular it suffices to prove the claim when each $a_{i}$ satisfies $a_{i}=2$. Proceeding by induction on $b_{2}(X)=n+3$ with $n \geq 1$, we get that

$$
\operatorname{det}\left(-Q_{X}\right)=4 \text {. }
$$

Since all principal minors are positive by induction, $-Q_{X}$ is positive definite.

\subsection{The realization problem: from correction terms to lattice theory}

In what follows, we will present the methodology we apply to prove Theorem 1.2. One main ingredient is the correction terms in Heegaard Floer homology.

In [17], Ozsváth and Szabó defined the correction term $d(Y, \mathfrak{t})$ that associates a rational number to an oriented rational homology sphere $Y$ equipped with a $\operatorname{Spin}^{c}$ structure $t$. They showed that this invariant obeys the relation

$$
d(-Y, \mathfrak{t})=-d(Y, \mathfrak{t}),
$$




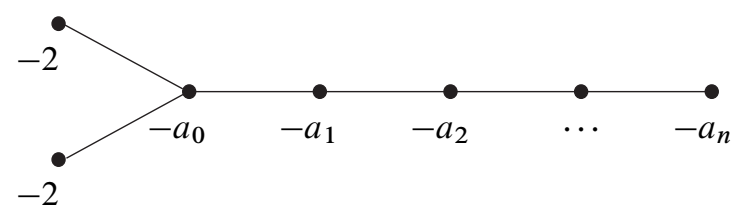

Figure 4: A negative definite plumbing diagram of $P(p, q)$.

where $-Y$ is the manifold $Y$ with the reversed orientation. If $Y$ is boundary of a negative definite four-manifold $X$, then

$$
c_{1}(\mathfrak{s})^{2}+b_{2}(X) \leq 4 d(Y, \mathfrak{t})
$$

for any $\mathfrak{s} \in \operatorname{Spin}^{c}(X)$ that extends $\mathfrak{t} \in \operatorname{Spin}^{c}(Y)$.

Definition 2.4 A smooth, compact, negative definite 4-manifold $X$ is sharp if for every $\mathfrak{t} \in \operatorname{Spin}^{c}(Y)$, there exists some $\mathfrak{s} \in \operatorname{Spin}^{c}(X)$ extending $\mathfrak{t}$ such that the equality is realized in equation (8).

The manifold $X=X(p, q)$ is an example of a sharp four-manifold. In order to prove this, it will be profitable to view the plumbing diagram of $X$, depicted in Figure 4, as a weighted graph, that is a graph equipped with an integer-valued function $m$ on its vertices. Given a weighted graph $G$, let $V$ be the abelian group freely generated by the vertices of $G$. Define a quadratic form

$$
Q_{G}: V \otimes V \rightarrow \mathbb{Z}
$$

as follows. For each vertex $v, Q_{G}(v \otimes v)=m(v)$; for each pair of distinct vertices $v$ and $w, Q_{G}(v \otimes w)$ is 1 if $v$ and $w$ are connected by an edge, and 0 otherwise.

Definition 2.5 A weighted graph $G$ is said to be a negative definite graph if

- $G$ is a disjoint union of trees, and

- the quadratic form associated to $G$ is negative definite.

The degree of a vertex $v$, denoted $d(v)$, is the number of edges incident to $v$. A vertex $v$ is said to be a bad vertex of the weighted graph if

$$
m(v)>-d(v) .
$$

Given a weighted graph $G$, we can get a four-manifold $X_{G}$ obtained from the plumbing construction instructed by $G$. In [18], Ozsváth and Szabó showed that if $G$ is a negative definite weighted graph with at most two bad vertices, then $X_{G}$ is sharp. In summary, using Lemma 2.3: 
Lemma 2.6 $X(p, q)$ is a sharp four-manifold.

We end this subsection by presenting how the integer surgery realization problem for prism manifolds translates to a lattice theory question.

Definition 2.7 A lattice is a finitely generated free abelian group $L$ together with a positive definite quadratic form

$$
\langle\cdot, \cdot\rangle: L \times L \rightarrow \mathbb{R}
$$

The lattice is integral if the value of the quadratic form is in $\mathbb{Z}$.

Throughout this paper, we will only consider integral lattices.

Definition 2.8 Suppose $p>1$ and $q<0$ are a pair of relatively prime integers. The $D$-type lattice $\Delta(p, q)$ is the lattice freely generated by elements

$$
x_{*}, x_{* *}, x_{0}, x_{1}, \ldots, x_{n}
$$

with inner product given by

$$
\left\langle x_{i}, x_{j}\right\rangle= \begin{cases}-1 & \text { if }\{i, j\} \text { is either }\{*, 0\} \text { or }\{* *, 0\}, \\ -1 & \text { if }|i-j|=1 \text { with } 0 \leq i, j \leq n, \\ 2 & \text { if } i=j \in\{*, * *\} \\ a_{i} & \text { if } 0 \leq i=j \leq n, \\ 0 & \text { in other cases, }\end{cases}
$$

where the coefficients $a_{0}, \ldots, a_{n}$ satisfy $a_{j} \geq 2$ and also (7). We then call (9) a vertex basis of $\Delta(p, q)$.

The inner product space $\left(H_{2}(X),-Q_{X}\right)$ equals $\Delta(p, q)$, where $X=X(p, q)$ is the four-manifold with Kirby diagram as in Figure 3. See also Figure 4. Now suppose that $4|q|$-surgery along a knot $K \subset S^{3}$ produces $P(p, q)$ with $q<0$. Let $W_{4|q|}$ denote the associated two-handle cobordism from $S^{3}$ to $P(p, q)$, capped off with $D^{4}$. Form the closed, oriented, smooth four-manifold $Z=X(p, q) \cup-W_{4|q|}$. Since $b_{1}\left(S_{4|q|}^{3}(K)\right)=0$, it follows that

$$
b_{2}(Z)=b_{2}(X)+b_{2}\left(-W_{4|q|}\right)=n+4 .
$$

The 4-manifold $-W_{4|q|}$ is negative definite. Using this together with Lemma 2.3 and the fact that $H_{2}(X) \oplus H_{2}\left(-W_{4|q|}\right) \hookrightarrow H_{2}(Z)$, it follows at once that $Z$ is negative definite. Using Lemma 2.6, and with the notation of this section in place, the following is immediate from [11, Theorem 3.3]. 
Theorem 2.9 Suppose $P(p, q)$ with $q<0$ arises from positive integer surgery on a knot in $S^{3}$. The D-type lattice $\Delta(p, q)$ is isomorphic to the orthogonal complement $(\sigma)^{\perp}$ of some changemaker vector $\sigma \in \mathbb{Z}^{n+4}$, and the generator of $H_{2}\left(W_{4|q|}\right)$ maps to $\sigma$.

Using techniques that will be developed in the next sections in tandem with Theorem 2.9, we will find a classification of all D-type lattices $\Delta(p, q)$ isomorphic to $(\sigma)^{\perp}$ for some changemaker vector $\sigma$ in $\mathbb{Z}^{n+4}$. If the corresponding prism manifold $P(p, q)$ does indeed arise from surgery on a knot $K \subset S^{3}$, we are able to compute the Alexander polynomial of $K$ from the values of the components of $\sigma$. Giving an algorithmic method to compute the coefficients of the polynomial occupies the rest of this section.

Let $\Sigma$ be the closed surface obtained from capping off a Seifert surface for $K$ in $W_{4|q|}$. It is straightforward to check that the class $[\Sigma]$ generates $H_{2}\left(W_{4|q|}\right)$. It follows from Theorem 2.9 that, under the embedding $H_{2}(X) \oplus H_{2}\left(-W_{4|q|}\right) \hookrightarrow H_{2}(Z)$, [ $\Sigma$ ] gets mapped to a changemaker vector $\sigma$. Let $\left\{e_{0}, e_{1}, \ldots, e_{n+3}\right\}$ be the standard orthonormal basis for $\mathbb{Z}^{n+4}$, and write

$$
\sigma=\sum_{i=0}^{n+3} \sigma_{i} e_{i}
$$

Also, define the characteristic covectors of $\mathbb{Z}^{n+4}$ to be

$$
\operatorname{Char}\left(\mathbb{Z}^{n+4}\right)=\left\{\sum_{i=0}^{n+3} \mathfrak{c}_{i} e_{i} \mid \mathfrak{c}_{i} \text { odd for all } i\right\} .
$$

We remind the reader that, writing the Alexander polynomial of $K$ as

$$
\Delta_{K}(T)=b_{0}+\sum_{i>0} b_{i}\left(T^{i}+T^{-i}\right),
$$

the $k^{\text {th }}$ torsion coefficient of $K$ is

$$
t_{k}(K)=\sum_{j \geq 1} j b_{k+j},
$$

where $k \geq 0$. With the preceding notation in place, the following lemma is immediate from [11, Lemma 2.5].

Lemma 2.10 The torsion coefficients satisfy

$$
t_{i}(K)= \begin{cases}\min _{\mathfrak{c}} \frac{1}{8}\left(\mathfrak{c}^{2}-n-4\right) & \text { for each } i \in\{0,1, \ldots, 2|q|\}, \\ 0 & \text { for } i>2|q|,\end{cases}
$$


where $\mathfrak{c}$ is subject to

$$
\mathfrak{c} \in \operatorname{Char}\left(\mathbb{Z}^{n+4}\right), \quad\langle\mathfrak{c}, \sigma\rangle+4|q| \equiv 2 i(\bmod 8|q|) .
$$

For $i>0$,

$$
b_{i}=t_{i-1}-2 t_{i}+t_{i+1} \quad \text { and } \quad b_{0}=1-2 \sum_{i>0} b_{i},
$$

where the $b_{i}$ are as in (11).

\section{D-type lattices}

Let $L$ be a lattice. Given $v \in L$, let $|v|=\langle v, v\rangle$ be the norm of $v$. Following Greene [10], say that an element $\ell \in L$ is reducible if $\ell=x+y$ for some nonzero $x, y \in L$ with $\langle x, y\rangle \geq 0$, and irreducible otherwise. We say $\ell$ is breakable if $\ell=x+y$ with $|x|,|y| \geq 3$ and $\langle x, y\rangle=-1$, and unbreakable otherwise.

The main goal of this section will be to characterize the irreducible and unbreakable elements of a D-type lattice $\Delta(p, q)$. Since any isomorphism of lattices must send irreducible elements to irreducible elements, and similarly unbreakables to unbreakables, this will let us constrain the form of an isomorphism between $\Delta(p, q)$ and a changemaker lattice.

The pairing graph, also introduced in [10], will be one of the main tools we use to study lattices:

Definition 3.1 Given a lattice $L$ and a subset $V \subset L$, the pairing graph is the graph $\widehat{G}(V)=(V, E)$, where $e=\left(v_{i}, v_{j}\right) \in E$ if $\left\langle v_{i}, v_{j}\right\rangle \neq 0$.

Proposition 3.2 For each $i \in\{*, * *, 0, \ldots, n\}, x_{i}$ is an irreducible element of $\Delta(p, q)$.

Proof Take some $w \in \Delta(p, q)$ with $w \neq 0, x_{i}$. Write

$$
w=w_{*} x_{*}+w_{* *} x_{* *}+\sum_{j=0}^{n} w_{j} x_{j}
$$

and consider $\left\langle w, x_{i}-w\right\rangle$. This is

$$
a_{i} w_{i}\left(1-w_{i}\right)-\sum_{j \neq i} a_{j} w_{j}^{2}+\left(\text { terms not involving the } a_{j}\right) .
$$

Here the $a_{j}$ are as defined in (7) when $j \geq 0$, and $\left\langle x_{j}, x_{j}\right\rangle=a_{j}$. We also let $a_{*}=a_{* *}=2$. The above expression is nonincreasing with respect to each $a_{j}$. Therefore, 
to show that $\left\langle w, x_{i}-w\right\rangle$ is negative, it suffices to show this when all of the $a_{j}$ are 2 . In this case, $\Delta(p, q)$ is isomorphic to the standard $D_{n+3}$ lattice, the lattice of elements of $\mathbb{Z}^{n+3}=\left\langle e_{-2}, e_{-1}, e_{0}, \ldots, e_{n}\right\rangle$ the sum of whose coefficients is even. This isomorphism sends

$$
x_{*} \mapsto e_{-2}+e_{-1}, \quad x_{* *} \mapsto-e_{-2}+e_{-1}, \quad x_{j} \mapsto-e_{j-1}+e_{j} \quad(j \geq 0) .
$$

If $\pm e_{i} \pm e_{j} \in \mathbb{Z}^{n+3}$ is written as a sum of two other elements of $\mathbb{Z}^{n+3}$ with nonnegative pairing, these must be $\pm e_{i}$ and $\pm e_{j}$. However, these are not in $D_{n+3}$. Therefore, the image of $x_{i}$ is an irreducible element of $D_{n+3}$, so $x_{i}$ is an irreducible element of $\Delta(p, q)$.

Corollary 3.3 The lattice $\Delta(p, q)$ is indecomposable, namely, $\Delta(p, q)$ is not the direct sum of two nontrivial lattices.

Proof Suppose that $\Delta(p, q) \cong L_{1} \oplus L_{2}$. Then each $x_{i}$, being irreducible, must be in either $L_{1}$ or $L_{2}$. However, any element of $L_{1}$ has zero pairing with any element of $L_{2}$. Since $x_{i} \sim x_{i+1}, \widehat{G}(\{*, * *, 0, \ldots, n\})$ is connected. This means that all of the $x_{i}$ are in the same part of the decomposition, and the other is trivial.

This gives us some information about irreducibility in the D-type lattices, but we want something more complete. Another important class of irreducible elements is that of the intervals:

Definition 3.4 For $A \subset\{*, * *, 0, \ldots, n\}$, let $[A]:=\sum_{i \in A} x_{i}$. We say $x \in \Delta(p, q)$ is an interval if $x=[A]$ for some $A$ where $\widehat{G}(A)$ is connected and $A$ does not contain both $*$ and $* *$.

Proposition 3.5 Intervals are irreducible.

Proof As in the proof of Proposition 3.2 we may assume that all of the $a_{i}=2$, and consider the image of an interval $x$ in $\mathbb{Z}^{n+3}$ under the embedding (12). (Note that the reduction to the case $a_{i}=2$ only works because all the coefficients $c_{i}$ of $x=\sum c_{i} x_{i}$ are 0 or \pm 1 .) Since $x$ is an interval, the image of $x$ is again of the form $\pm e_{i} \pm e_{j}$, which is irreducible as in Proposition 3.2.

For linear lattices, this is essentially the end of the story - Greene proves that every irreducible is either an interval or the negation of an interval. The situation here is somewhat richer, but is similar in spirit: every irreducible is either an interval, or can 
be obtained from one by applying some involution of the lattice. To prove this, we first need to know a few involutions of $\Delta(p, q)$.

Definition 3.6 Let $m$ be the smallest index for which $a_{m} \geq 3$. For any $0 \leq j \leq m$, let $y_{j}=x_{*}+x_{* *}+2 \sum_{0 \leq i<j} x_{i}$, and let $\tau_{j}: \Delta(p, q) \rightarrow \Delta(p, q)$ be the reflection in the subspace spanned by $y_{0}, \ldots, y_{j}$. More explicitly, $\tau_{j}$ acts as

$$
\tau_{j}\left(x_{i}\right)= \begin{cases}x_{* *} & \text { if } i=*, \\ x_{*} & \text { if } i=* *, \\ x_{i} & \text { if } 0 \leq i<j, \\ -x_{*}-x_{* *}-2 x_{0}-\cdots-2 x_{j-1}-x_{j} & \text { if } i=j, \\ -x_{i} & \text { if } i>j .\end{cases}
$$

Lemma 3.7 Each $\tau_{j}$ satisfies $\left\langle\tau_{j}(x), \tau_{j}(y)\right\rangle=\langle x, y\rangle$ and $\tau_{j}^{2}(x)=x$. Furthermore, for any $v, w \in \Delta(p, q),\langle v, w\rangle \equiv\left\langle\tau_{j}(v), w\right\rangle(\bmod 2)$.

Proof Each $\tau_{j}$ is a reflection, which gives the first two properties. For the last property, it suffices to check that $\left\langle\tau_{j}\left(v_{i}\right), v_{k}\right\rangle \equiv\left\langle v_{i}, v_{k}\right\rangle(\bmod 2)$ for all $i, j, k$, which is entirely straightforward.

The following special case of Lemma 3.7 will be used very heavily in what follows:

Lemma 3.8 If $x \in \Delta(p, q)$, then $\left\langle x, x_{*}\right\rangle \equiv\left\langle x, x_{* *}\right\rangle(\bmod 2)$.

Proof This follows from Lemma 3.7 since $x_{* *}=\tau_{0}\left(x_{*}\right)$.

The $\tau_{j}$ preserve the pairings on $\Delta(p, q)$, so $\tau_{j}(x)$ is irreducible whenever $x$ is. This gives some noninterval examples of irreducible elements of $\Delta(p, q)$. For example,

$$
x_{*}+x_{* *}+x_{0}+\cdots+x_{i}=-\tau_{0}\left(x_{0}+\cdots+x_{i}\right)
$$

and (as long as $m \geq 2$ )

$$
x_{*}+x_{* *}+2 x_{0}+2 x_{1}+x_{2}+\cdots+x_{i}=-\tau_{2}\left(x_{2}+\cdots+x_{i}\right)
$$

are both irreducible. However, these are essentially the only examples:

Proposition 3.9 If $x \in \Delta(p, q)$ is any irreducible element, either $x$ or $-x$ is an interval, or there is some $j$ such that either $\tau_{j}(x)$ or $-\tau_{j}(x)$ is an interval. 
Proof Suppose $x=\sum c_{i} x_{i}$ is irreducible. Replacing $x$ by $-x$ if necessary, we can assume that $c_{0} \geq 0$ and there is at least one $c_{i}>0$. Let $A \subset\{*, * *, 0, \ldots, n\}$ be the set of indices $i$ with $c_{i}<0$, and let $y=\sum_{i \in A} c_{i} x_{i}$. Clearly, $\langle y, x-y\rangle$ is a sum of terms of the form $-c_{i} c_{i^{\prime}}$ with $c_{i}<0$ and $c_{i^{\prime}} \geq 0$, so is nonnegative. Since $x$ is irreducible, then $y=0$, so actually all of the $c_{i}$ are nonnegative.

Now, let $B \subset\{*, * *, 0, \ldots, n\}$ be the set of indices $i$ with $c_{i} \neq 0$. The pairing graph $\widehat{G}(B)$ must be connected, since otherwise $x$ could be written as a sum of two nonzero elements that pair to zero.

If $c_{i} \in\{0,1\}$ for all $i$, then $x=[B]$. Since $\widehat{G}(B)$ is connected, either $x$ is already an interval, or $B=\{*, * *, 0, \ldots, j\}$ for some $j$, but then $-\tau_{0}(x)=x_{0}+\cdots+x_{j}$ is an interval.

If $c_{i}>1$ for some $i$, let $z=[B]$, and consider the pairing $\langle z, x-z\rangle$. If $0 \notin B$, then let $j_{0}=\min B$ and $j_{1}=\max B$, so

$$
\langle z, x-z\rangle=\left(\sum_{j_{0} \leq i \leq j_{1}}\left(a_{i}-2\right)\left(c_{i}-1\right)\right)+c_{j_{0}}+c_{j_{1}}-2 .
$$

Since $c_{i} \geq 1$ for each $i \in B$, and $a_{i} \geq 2$ for each $i$, this is nonnegative, contradicting the irreducibility of $x$. Therefore, $0 \in B$, so $c_{0} \geq 1$. Since $\widehat{G}(B)$ is connected, then, $c_{i} \geq 1$ for all $0 \leq i \leq \max B$.

Now let $j$ be the largest index with $c_{j}>1$. Suppose there were some largest $k$, $0 \leq k<j$, with $c_{k}<c_{j}$, and let $w=x_{k+1}+\cdots+x_{j}$. Then

$$
\langle w, x-w\rangle=\left(\sum_{k+1 \leq i \leq j}\left(a_{i}-2\right)\left(c_{i}-1\right)\right)+\left(c_{k+1}-c_{k}\right)+\left(c_{j}-c_{j+1}\right)-2 .
$$

Since all of the $c_{i}$ are at least 1 , each of these terms is nonnegative, so this cannot happen. Therefore, $c_{i} \geq c_{j}>1$ for any $0 \leq i<j$.

Finally, let $w_{0}=x_{0}+\cdots+x_{j}$, and let $w_{1}=x_{*}+x_{* *}+w_{0}$. Consider

$$
\begin{aligned}
& \left\langle w_{0}, x-w_{0}\right\rangle=\left(\sum_{0 \leq i \leq j}\left(a_{i}-2\right)\left(c_{i}-1\right)\right)-\left(c_{*}+c_{* *}-c_{0}\right)+\left(c_{j}-2\right)-c_{j+1}, \\
& \left\langle w_{1}, x-w_{1}\right\rangle=\left(\sum_{0 \leq i \leq j}\left(a_{i}-2\right)\left(c_{i}-1\right)\right)+\left(c_{*}+c_{* *}-c_{0}\right)+\left(c_{j}-2\right)-c_{j+1} .
\end{aligned}
$$

The first term of each of these sums is nonnegative and, for at least one of these sums, the second term is nonnegative. Moreover, $c_{j} \geq 2$, so the third term is also nonnegative. 
The last term $c_{j+1}$ is 0 or 1 . Therefore, for both of these to be negative, the first three terms must be zero, and the last one must be negative. Since $c_{i}>1$ for $i \leq j$, the first term can only be zero when $a_{i}=2$ for $0 \leq i \leq j$, so $j<m$, where $m$ is as in Definition 3.6. For the remaining terms to be zero or negative, $c_{j}=2, c_{0}=c_{*}+c_{* *}$, and $c_{j+1}=1$.

Since $j<m$, we can consider $-\tau_{j+1}(x)=\sum c_{i}^{\prime} x_{i}$. For $i>j, c_{i}^{\prime}=c_{i} \leq 1$ and $c_{j}^{\prime}=2 c_{j+1}-c_{j}=0$. Note that $c_{j+1}^{\prime}=c_{j+1}=1 \neq 0$. So $c_{i}^{\prime}$ vanishes for $i<j$ as well, since $-\tau_{j+1}(x)$ is still irreducible. Therefore, $-\tau_{j+1}(x)=\sum_{i>j} c_{i} x_{i}$, which is an interval.

We will also want to know which irreducible elements of $\Delta(p, q)$ are breakable. Since negation and the $\tau_{j}$ preserve breakability, it will suffice to know this for intervals:

Lemma 3.10 An interval $x=[A]$ is breakable if there are at least two indices $i, k \in A$ with $a_{i}, a_{k} \geq 3$.

Proof Suppose that $i, k \in A$ with $a_{i}, a_{k} \geq 3$. Fix some index $J$ with $i \leq J<k$, and let $T_{0}=\{j \in A \mid j \leq J\}$ and $T_{1}=\{j \in A \mid J<j\}$. Since $i \in T_{0}$ and $k \in T_{1}$, $\left|\left[T_{0}\right]\right| \geq 3$ and $\left|\left[T_{1}\right]\right| \geq 3$. Also, since $x$ is an interval, $j \in A$ for all $i \leq j \leq k$, so $J \in T_{0}$ and $J+1 \in T_{1}$. Therefore, $\left\langle\left[T_{0}\right],\left[T_{1}\right]\right\rangle=-1$, and $x$ is breakable.

Definition 3.11 When $\left[A_{j}\right]$ is unbreakable and has norm at least 3 , let $z_{j} \in A_{j}$ be the unique element with $\left|z_{j}\right| \geq 3$.

Finally, let us determine when two D-type lattices are isomorphic.

Proposition 3.12 If $\Delta(p, q) \cong \Delta\left(p^{\prime}, q^{\prime}\right)$, then $p=p^{\prime}$ and $q=q^{\prime}$.

Proof Suppose $L$ is a lattice isomorphic to $\Delta(p, q)$ for some $p$ and $q$. To recover $p$ and $q$ from $L$, it suffices to recover the ordered sequence of norms $\left(\left|x_{0}\right|,\left|x_{1}\right|, \ldots,\left|x_{n}\right|\right)$ of the vertex basis, or equivalently to recover the weighted tree $\mathcal{T}$ corresponding to $\Delta(p, q)$. As in the proof of [10, Proposition 3.6], we will first recover the vertices with weight at least 3 , and then fill in the vertices of weight 2 . Let $R \subset L$ be the sublattice generated by the vectors of norm 2 in $L$. Every such element is irreducible as in Proposition 3.2. It then follows from Proposition 3.9 that $R$ is generated by vertices with weight 2 , since the involution $\tau_{j}$ does not change the norm, and that an interval of norm 2 merely has vertices of norm 2 . 
Let $V_{0}$ be the set of irreducible, unbreakable elements of $L$ of norm at least 3, and let $V$ be the quotient of $V_{0}$ by the equivalence $v \sim u$ whenever either $v-u \in R$ or $v+u \in R$. By Proposition 3.9 and Lemma 3.10, each irreducible, unbreakable element of $L$ corresponds (up to sign and applications of the $\tau_{j}$ ) to an interval containing a unique vertex of weight at least 3 . We claim that the set $V$ is in bijection with the vertices in $\mathcal{T}$ of weight at least 3 . In fact, if $v-u$ or $v+u$ can be written as a sum of vectors of norm 2 , the corresponding high-weight vertices must be the same: if we write $v=\sum v_{i} x_{i}$ and $\pm \tau_{j}(v)=\sum v_{i}^{\prime} x_{i}$ (where $\pm \tau_{j}(v)$ is an interval), then the coefficient $v_{i}^{\prime}$ is nonzero for exactly one $i$ with $\left|x_{i}\right| \geq 3$. Since applying any $\tau_{j}$ does not change the coefficient $v_{i}$ when $\left|x_{i}\right| \geq 3$ except by a sign, the original coefficient $v_{i}$ is also nonzero (in fact, \pm 1 ) for exactly one $i$ with $\left|x_{i}\right| \geq 3$. Similarly, there is exactly one $j$ with $\left|x_{j}\right| \geq 3$ with $u_{j}$ nonzero and equal to \pm 1 , where $u=\sum u_{j} x_{j}$. Therefore, one of $v-u$ or $v+u$ can be written as a sum of vertices of weight 2 if and only if the high-weight vertices are the same. This finishes the proof of the claim. Also, if $v \in L$ is a representative of some class in $V$, then $|v|$ is the weight of the corresponding vertex.

Let $W$ be the set of indecomposable sublattices of $R$. Each element of $W$ corresponds to a connected subgraph of $\mathcal{T}$, all of whose vertices have weight 2 . As a lattice, each element $w \in W$ is isomorphic to either the root lattice $A_{n_{w}}$ for some $n_{w}$, or to $D_{n_{w}}$ if the corresponding subgraph contains all of $x_{*}, x_{* *}, x_{0}$ and $x_{1}$. This last case happens for at most one $w \in W$. Now, form a bipartite graph $\mathcal{B}$ as follows: the vertex set is $V \cup W$, and there is an edge between $v \in V$ and $w \in W$ if, for some representative $\widetilde{v} \in L$ of $v$ and some element $\widetilde{w} \in w,\langle\widetilde{v}, \widetilde{w}\rangle \neq 0$. This happens if and only if there is an edge in $\mathcal{T}$ between the vertex corresponding to $v$ and some vertex of the subgraph corresponding to $w$. In $\mathcal{B}$, each $w \in W$ neighbors at most two vertices $v \in V$, or at most one if the corresponding sublattice is isomorphic to $D_{n_{w}}$.

We say an edge connecting $v \in V$ and $w \in W$ is special if $w$ is isomorphic to $A_{3}$, and there exists a representative $\tilde{v}$ of $v$ such that there exist exactly 4 vectors $\tilde{w} \in w$ satisfying $|\widetilde{w}|=2$ and $\langle\widetilde{v}, \widetilde{w}\rangle=-1$. It is easy to check that an edge is special if and only if $a_{0}=2, a_{1}>2, w$ is generated by $x_{*}, x_{* *}$ and $x_{0}$, and $v$ corresponds to $x_{1}$.

The graph $\mathcal{B}$ contains all of the information about how the blocks of vertices of weight 2 fit together with the other vertices in $\mathcal{T}$. Since the part of $\mathcal{T}$ corresponding to one of those blocks must be the Dynkin diagram of the corresponding sublattice, reconstructing $\mathcal{T}$ is straightforward. The vertex set of $\mathcal{T}$ is a copy of $V$ together with a copy of $V_{w}$ for each $w \in W$, where $V_{w}$ is a set containing $\operatorname{rank}(w)$ vertices. The edges 
in $\mathcal{T}$ are constructed as follows. If the sublattice $w$ is isomorphic to $A_{n_{w}}$, connect the vertices in $V_{w}$ together in a path $P_{w}$. For each nonspecial edge in $\mathcal{B}$ connecting $v$ and $w$, connect $v$ to one of the ends of $P_{w}$. For each $w$, at most two vertices in $V$ need to be connected in this way, so they can always be connected so that no end of $P_{w}$ has valency 3 in $\mathcal{T}$. If there is a special edge between $v$ and $w$, connect the middle vertex of $P_{w}$ to $v$. If the sublattice $w$ is isomorphic to $D_{n_{w}}$, connect $n_{w}-2$ of the vertices in $V_{w}$ into a path, connect the remaining two to one end of the path, and if there is a $v \in V$ neighboring $w$ in $\mathcal{B}$, connect it to the other end. Finally, for $v, v^{\prime} \in V$, put an edge between them if there are representatives $\widetilde{v}, \widetilde{v}^{\prime}$ with $\left\langle\widetilde{v}, \widetilde{v}^{\prime}\right\rangle \neq 0$ and $v, v^{\prime}$ do not have a common neighbor in $\mathcal{B}$. For the weights, put a 2 on any vertex in $V_{w}$, and $|\widetilde{v}|$ on a vertex $v \in V$, where $\widetilde{v}$ is a representative of the class $v$.

\section{Changemaker lattices}

According to Theorem 2.9, whenever $P(p, q)$ comes from positive integer surgery on a knot, $\Delta(p, q)$ is isomorphic to the orthogonal complement $(\sigma)^{\perp}$ for some changemaker vector $\sigma \in \mathbb{Z}^{n+4}$. A lattice is called a changemaker lattice if it is isomorphic to the orthogonal complement of a changemaker vector. In this section, we will prove some basic structural results about D-type lattices which are also changemaker lattices.

Write $\left(e_{0}, e_{1}, \ldots, e_{n+3}\right)$ for the orthonormal basis of $\mathbb{Z}^{n+4}$, and write $\sigma=\sum_{i} \sigma_{i} e_{i}$. Since $\Delta(p, q)$ is indecomposable (Corollary 3.3), $\sigma_{0} \neq 0$, otherwise $(\sigma)^{\perp}$ would have a direct summand $\mathbb{Z}$. So $\sigma_{0}=1$.

We will need several results from [10, Section 3] about changemaker lattices:

Definition 4.1 [10, Definition 3.11] The standard basis of $(\sigma)^{\perp}$ is the collection $S=\left\{v_{1}, \ldots, v_{n+3}\right\}$, where

$$
v_{j}=\left(2 e_{0}+\sum_{i=1}^{j-1} e_{i}\right)-e_{j}
$$

whenever $\sigma_{j}=1+\sigma_{0}+\cdots+\sigma_{j-1}$, and

$$
v_{j}=\left(\sum_{i \in A} e_{i}\right)-e_{j}
$$

whenever $\sigma_{j}=\sum_{i \in A} \sigma_{i}$, with $A \subset\{0, \ldots, j-1\}$ chosen to maximize the quantity $\sum_{i \in A} 2^{i}$. A vector $v_{j} \in S$ is called tight in the first case, just right in the second case 
as long as $i+1 \in A$ whenever $i<j-1$ and $i \in A$, and gappy if there is some index $i$ with $i \in A, i<j-1$, and $i+1 \notin A$. In this situation, call $i$ a gappy index for $v_{j}$.

Definition 4.2 For $v \in \mathbb{Z}^{n+4}, \operatorname{supp} v=\left\{i \mid\left\langle e_{i}, v\right\rangle \neq 0\right\}$ and $\operatorname{supp}^{+} v=\left\{i \mid\left\langle e_{i}, v\right\rangle>0\right\}$.

Lemma 4.3 [10, Lemma 3.12(3)] If $\left|v_{k+1}\right|=2$, then $k$ is not a gappy index for any $v_{j} \in S$.

Proof This follows from the maximality of the set $A$ : if this did not hold, we could remove $k$ from $A$ and add $k+1$, increasing the sum $\sum_{i \in A} 2^{i}$ but leaving the sum $\sum_{i \in A} \sigma_{i}$ unchanged.

Lemma 4.4 [10, Lemma 3.13] Each $v_{j} \in S$ is irreducible.

Lemma 4.5 [10, Lemma 3.15] If $v_{j} \in S$ is breakable, it is tight.

Lemma 4.6 [10, Lemma 3.14(2)] If $v_{t} \in S$ is tight, $j>t$ and $v_{j}=e_{t}+e_{j-1}-e_{j}$, then $v_{t}+v_{j}$ is irreducible.

Lemma 4.7 If $v_{t} \in S$ is tight, $j>t$ and $v_{j}=e_{0}+\cdots+e_{t-1}+\left(\sum_{i \in A} e_{i}\right)-e_{j}$ for some $A \subseteq\{t+1, \ldots, j-1\}$, then $v_{j}-v_{t}$ is irreducible.

Proof For contradiction, suppose there exist $x$ and $y$ with

$$
v_{j}-v_{t}=-e_{0}+e_{t}+\left(\sum_{i \in A} e_{i}\right)-e_{j}=x+y
$$

such that $\langle x, y\rangle \geq 0$. Write $x=\sum x_{i} e_{i}$ and $y=\sum y_{i} e_{i}$. Since $\left|x_{i}+y_{i}\right| \leq 1$ for all $i$, $x_{i} y_{i} \leq 0$ for all $i$. Since $\langle x, y\rangle=\sum x_{i} y_{i} \geq 0$, we must have $x_{i} y_{i}=0$ for each $i$. Observe only $e_{0}$ and $e_{j}$ have negative coefficients in $v_{j}-v_{t}$. If $x_{0}=x_{j}=0$ (resp. $y_{0}=y_{j}=0$ ), then since $x$ (resp. $y$ ) is in $\langle\sigma\rangle^{\perp}$ and has nonnegative coefficients, $x=0$ (resp. $y=0$ ). If $x_{0}=0$ and $x_{j}=-1$, since $\sum \sigma_{i} y_{i}=0, y=e_{s}-e_{0}$ for some $0<s<t$. Since $y=v_{j}-v_{t}-x, x_{s}=-1$ and $x_{s} y_{s} \neq 0$, a contradiction. If $x_{0}=-1$ and $x_{j}=0$, we get a contradiction just as in the previous case.

If we have a lattice $L$ isomorphic to both a D-type lattice $\Delta(p, q)$ and a changemaker lattice $(\sigma)^{\perp}$, it has both the vertex basis $x_{*}, x_{* *}, x_{0}, \ldots, x_{n}$ and the standard basis $v_{1}, \ldots, v_{n+3}$. Since each of the $v_{i}$ is irreducible, we can use Proposition 3.9 to constrain its expression in terms of the $x_{i}$. In some cases, we can say more: 
Lemma 4.8 If $\Delta(p, q)$ is isomorphic to $(\sigma)^{\perp}$ for some changemaker $\sigma \in \mathbb{Z}^{n+4}$, then there is an isomorphism $\phi: \Delta(p, q) \stackrel{\sim}{\rightarrow}(\sigma)^{\perp}$ that sends $x_{*}$ to $v_{1}$ and $x_{* *}$ to $v_{f}$ for some index $f$. Furthermore, if $a_{0}=2$, then $f=3$ and we can also choose $\phi$ to send $x_{0}$ to $v_{2}$.

Proof Let $\phi_{0}: \Delta(p, q) \rightarrow(\sigma)^{\perp}$ be an isomorphism. Both $x_{*}$ and $x_{* *}$ have norm 2, and $\left\langle x_{*}, x_{* *}\right\rangle=0$. The only elements of $(\sigma)^{\perp}$ of norm 2 have the form $e_{i}-e_{j}$ for indices $i$ and $j$ with $\sigma_{i}=\sigma_{j}$. Since the entries of $\sigma$ are nondecreasing, actually $\sigma_{i}=\sigma_{k}=\sigma_{j}$ for any $\min (i, j) \leq k \leq \max (i, j)$, so in particular $\sigma_{i}=\sigma_{j}=\sigma_{m}=\sigma_{m+1}$ for $m=\min (i, j)$. We define an automorphism $\psi$ of $\mathbb{Z}^{n+4}$ as follows. If $|j-i|=1$, let

$$
\psi\left(e_{i}\right)=e_{m}, \quad \psi\left(e_{j}\right)=e_{m+1}, \quad \psi\left(e_{l}\right)=e_{l} \quad \text { when } l \notin\{i, j\} .
$$

If $|j-i|>1$, then $\psi\left(e_{i}\right)$ and $\psi\left(e_{j}\right)$ are as above, and

$$
\psi\left(e_{m+1}\right)=e_{\max (i, j)}, \quad \psi\left(e_{l}\right)=e_{l} \quad \text { when } l \notin\{i, j, m+1\} .
$$

Since $\psi$ fixes $\sigma$, it restricts to an automorphism $\bar{\psi}$ of $(\sigma)^{\perp}$. Consider $\bar{\psi} \circ \phi_{0}$, which sends $\phi_{0}^{-1}\left(e_{i}-e_{j}\right)$ to the standard basis vector $v_{m+1}$. Since $\left\langle x_{*}, x_{* *}\right\rangle=0$, this process can be done separately for $x_{*}$ and $x_{* *}$, making them both sent to standard basis vectors $v_{e}, v_{f}$. By precomposing with the automorphism of $\Delta(p, q)$ that switches $x_{*}$ and $x_{* *}$, we can assume $e<f$, and then Lemma 3.8 ensures that $e=1$, because otherwise $\left\langle v_{e-1}, x_{*}\right\rangle=-1$ while $\left\langle v_{e-1}, x_{* *}\right\rangle=0$.

When $a_{0}=2, x_{0}$ has norm 2 and pairing -1 with both $x_{*}$ and $x_{* *}$, so $x_{0}=e_{1}-e_{f-1}$ or $-e_{0}+e_{f}$. It follows that $\sigma_{0}=\sigma_{1}=\cdots=\sigma_{f-1}=\sigma_{f}$. Therefore, $v_{2}=e_{1}-e_{2}$, so $\left\langle v_{2}, v_{1}\right\rangle=-1$. By Lemma 3.8, $\left\langle v_{2}, v_{f}\right\rangle$ is odd. This can happen only if $f=3$, so $v_{f}=e_{3}-e_{2}$, and $x_{0}=e_{1}-e_{2}$ or $-e_{0}+e_{3}$. Since $\sigma_{0}=\sigma_{1}=\sigma_{2}=\sigma_{3}$, any permutation of $\{0,1,2,3\}$ induces a permutation of $\left\{e_{0}, e_{1}, e_{2}, e_{3}\right\}$, hence gives an automorphism of $(\sigma)^{\perp}$. If $x_{0}=e_{1}-e_{2}$, we are done. If $x_{0}=-e_{0}+e_{3}$, we can precompose $\phi_{0}$ with the automorphism exchanging $x_{*}$ and $x_{* *}$, and postcompose $\phi_{0}$ with the automorphism given by the permutation (02)(13).

From now on, let $L=(\sigma)^{\perp}$ be a changemaker lattice isomorphic to some D-type lattice, and identify $x_{*}, x_{* *}, x_{0}, \ldots, x_{n}$ with elements of $L$ according to an isomorphism chosen as in Lemma 4.8. Since $\sigma_{0}=1$, by Lemma 4.8, we see that

$$
\sigma_{1}=\sigma_{0}=1
$$


Lemma 4.9 For any $v_{j} \in S$, we have $j-1 \in \operatorname{supp} v_{j}$.

Proof This is just the second part of [10, Lemma 3.12(1)], plus the fact that $v_{1}=$ $e_{0}-e_{1}$.

Lemma 4.10 If $a_{0}=2$, then $a_{1} \geq 3$.

Proof Suppose $a_{0}=2$. Note that $x_{1}$ satisfies $\left\langle x_{1}, x_{0}\right\rangle=-1$ and $\left\langle x_{1}, x_{*}\right\rangle=$ $\left\langle x_{1}, x_{* *}\right\rangle=0$. Therefore, if we write $x_{1}=\sum_{i=0}^{n+3} c_{i} e_{i}$, then $c_{0}=c_{1}=c_{2}-1=c_{3}-1$. Also, $x_{1}$ is not in the span of $x_{0}, x_{*}$ and $x_{* *}$, so there is some $j>3$ with $c_{j} \neq 0$. Therefore, at least three of the $c_{i}$ are nonzero, so $a_{1}=\left|x_{1}\right| \geq 3$.

In particular, this means that $m \leq 1$ in the notation of Definition 3.6. So we only ever need to use the involution $\tau_{j}$ for $j \in\{0,1\}$ to make irreducible elements into intervals.

Lemma 4.11 If $v_{j}$ is a standard basis vector for $j \neq 1, f$ and $v_{j}$ is not tight, then $\left\langle v_{j}, v_{1}\right\rangle=\left\langle v_{j}, v_{f}\right\rangle \in\{-1,0\}$. If $v_{j}$ is tight, $j=f-1$ and $\left\langle v_{j}, v_{1}\right\rangle=-\left\langle v_{j}, v_{f}\right\rangle=1$.

Proof Using Lemma 4.3, supp $v_{j} \cap\{0,1\} \neq\{0\}$, so $\left\langle v_{j}, v_{1}\right\rangle \in\{0,-1\}$ unless $v_{j}$ is tight. Similarly, either $j \neq f-1$ and supp $v_{j} \cap\{f-1, f\} \neq\{f-1\}$, or $j=f-1$ and supp $v_{j} \cap\{f-1, f\}=\{f-1\}$. In either case, $\left\langle v_{j}, v_{f}\right\rangle \in\{0,-1\}$. By Lemma 3.8, these two values have the same parity, so they must be equal if $v_{j}$ is not tight. If $v_{j}$ is tight, $\left\langle v_{j}, v_{1}\right\rangle$ is 1 , so $\left\langle v_{j}, v_{f}\right\rangle$ is -1 . Therefore, $j=f-1$.

Corollary 4.12 There is at most one tight vector in $S$. If $a_{0}=2$, there is no tight vector.

Proposition 4.13 If $a_{0} \geq 3$, then for any standard basis vector $v_{j}$, either $v_{j}$ is an interval or $\tau_{0}\left(v_{j}\right)$ is. If $a_{0}=2$, the same conclusion holds with $\tau_{1}$ instead of $\tau_{0}$. Furthermore, this interval contains $*$ or $* *$ if and only if $j$ is 1 or $f$ or $v_{j}$ is tight. If $a_{0}=2$, then this interval contains 0 if and only if $j=2$.

Proof First, suppose that $a_{0} \geq 3$, so $m=0$ and Proposition 3.9 says that one of $v_{j}$, $-v_{j}, \tau_{0}\left(v_{j}\right)$ or $-\tau_{0}\left(v_{j}\right)$ is an interval. It remains only to show that we do not ever need to use a negation. If $j \in\{1, f\}, v_{j} \in\left\{x_{*}, x_{* *}\right\}$ is an interval. Therefore, suppose $j \neq 1, f$. If $v_{j}$ is tight, by Lemma $4.11\left\langle v_{j}, y_{0}\right\rangle=0$, where $y_{0}=x_{*}+x_{* *}$ is defined in Definition 3.6. Since $\tau_{0}$ is the reflection in the line spanned by $y_{0}$, we get that 
$\tau_{0}\left(v_{j}\right)=-v_{j}$ and $-\tau_{0}\left(v_{j}\right)=v_{j}$, and we are done because we do not need to use the negation or $-\tau_{0}$. If $v_{j}$ is not tight, Lemma 4.11 says that $\left\langle v_{j}, x_{*}\right\rangle=\left\langle v_{j}, x_{* *}\right\rangle \in\{0,-1\}$. If $\left\langle v_{j}, x_{*}\right\rangle=0$, we get $\left\langle v_{j}, y_{0}\right\rangle=0$. We are done by the same argument as before. If $\left\langle v_{j}, x_{*}\right\rangle=\left\langle v_{j}, x_{* *}\right\rangle=-1$, we have

$$
\left\langle\tau_{0}\left(v_{j}\right), x_{*}\right\rangle=\left\langle\tau_{0}\left(v_{j}\right), \tau_{0}\left(x_{* *}\right)\right\rangle=\left\langle v_{j}, x_{* *}\right\rangle=-1
$$

and similarly $\left\langle\tau_{0}\left(v_{j}\right), x_{* *}\right\rangle=-1$. Therefore, if $-v_{j}$ or $-\tau_{0}\left(v_{j}\right)$ were an interval $[A]$, it would satisfy

$$
\left\langle[A], x_{*}\right\rangle=\left\langle[A], x_{* *}\right\rangle=1 .
$$

This can only happen if $*, * *$ and 0 are all in $A$, so $[A]$ is not an interval.

If $a_{0}=2$, then $\left(v_{1}, v_{2}, v_{3}\right)=\left(x_{*}, x_{0}, x_{* *}\right)$, all of which are intervals, so we can assume $j>3$. By Corollary 4.12, $v_{j}$ is not tight, so Lemma 4.11 implies that $\left\langle v_{j}, v_{1}\right\rangle=$ $\left\langle v_{j}, v_{3}\right\rangle$. Since $\left|v_{1}\right|=\left|v_{2}\right|=\left|v_{3}\right|=2$, Lemma 4.3 implies that supp $v_{j} \cap\{0,1,2,3\}$ is either $\{0,1,2,3\},\{2,3\}$ or $\varnothing$. In any case, $\left\langle v_{j}, v_{1}\right\rangle=\left\langle v_{j}, v_{3}\right\rangle=0$. Therefore, $\tau_{0}\left(v_{j}\right)=-v_{j}$. This means that, for $j>3$, one of $v_{j},-v_{j}, \tau_{1}\left(v_{j}\right)$ or $-\tau_{1}\left(v_{j}\right)$ must be an interval. We can assert that 0 is never in this interval, since $v_{j}$ has zero pairing with $v_{1}=x_{*}$ and $v_{3}=x_{* *}$. If 1 is not in this interval, then also $\left\langle v_{j}, x_{0}\right\rangle=0$, so $\left\langle v_{j}, y_{1}\right\rangle=0$. Since $\tau_{1}$ is the reflection in the plane spanned by $y_{0}, y_{1}, \tau_{1}\left(v_{j}\right)=-v_{j}$, and we are done. It remains only to show that if $[A]$ is an interval containing 1 but not 0 , then neither $-[A]$ nor $-\tau_{1}([A])$ can be a standard basis vector, which holds because in this case

$$
\left\langle-[A], x_{0}\right\rangle=\left\langle-\tau_{1}([A]), x_{0}\right\rangle=1,
$$

and by Lemma 4.11 for any nontight standard basis vector this pairing is either 0 or -1 .

From now on, let $\tau$ denote $\tau_{0}$ if $a_{0} \geq 3$, or $\tau_{1}$ if $a_{0}=2$. For each standard basis vector $v_{j}$, let $\left[A_{j}\right]$ be an interval for which either $v_{j}=\left[A_{j}\right]$ or $v_{j}=\tau\left(\left[A_{j}\right]\right)$, and let $\epsilon_{j}=1$ if $v_{j}=\left[A_{j}\right]$, and -1 otherwise. When $a_{0} \geq 3$, for all $j$ except 1 and $f$, $\epsilon_{j}$ is uniquely determined; when $a_{0}=2$, for $j \neq 1,2,3, \epsilon_{j}$ is uniquely determined. However, $\epsilon_{1}$ and $\epsilon_{f}$ can be freely chosen since $\tau$ exchanges $v_{1}$ and $v_{f}$. To resolve this ambiguity, we will choose $\left[A_{1}\right]$ and $\left[A_{f}\right]$ to make $\epsilon_{1}=-\epsilon_{f}=\epsilon_{f-1}$, and, when $a_{0}=2$, choose $\epsilon_{2}=1$. This makes $A_{1}=A_{f}$, and, when $v_{f-1}$ is tight, $A_{1}=A_{f}=\{*\}$ whenever $* \in A_{f-1}$, and $\{* *\}$ otherwise. By Proposition 4.13, this means that either $*$ or $* *$ is in none of the $A_{j}$, which gives the following fact: 
Lemma 4.14 If $A_{j}$ and $A_{k}$ are disjoint, $\left\langle\left[A_{j}\right],\left[A_{k}\right]\right\rangle$ is either 0 or -1 . Otherwise, $\left\langle\left[A_{j}\right],\left[A_{k}\right]\right\rangle$ is either $\left|\left[A_{j} \cap A_{k}\right]\right|-1$ or $\left|\left[A_{j} \cap A_{k}\right]\right|-2$, where $\left|\left[A_{j} \cap A_{k}\right]\right|$ is either $\left|\left[A_{j}\right]\right|$ or 2 if $\left[A_{j}\right]$ is unbreakable.

Proof For simplicity in this proof, call whichever of $*$ or $* *$ occurs in some of the $A_{j}-1$. If $A_{j}$ and $A_{k}$ are disjoint, either there is no index $i$ with $x_{i} \in A_{j}$ and $x_{i \pm 1} \in A_{k}$, in which case they pair to 0 , or there is exactly one, in which case they pair to -1 . If $A_{j}$ and $A_{k}$ do have nonempty intersection, either $\min A_{j}=\min A_{k}$ or max $A_{j}=\max A_{k}$, in which case $\left\langle\left[A_{j}\right],\left[A_{k}\right]\right\rangle$ is $\left|\left[A_{j} \cap A_{k}\right]\right|-1$, or this does not hold, in which case $\left\langle\left[A_{j}\right],\left[A_{k}\right]\right\rangle=\left|\left[A_{j} \cap A_{k}\right]\right|-2$. To compute $\left|\left[A_{j} \cap A_{k}\right]\right|$, note that for any $A \subset\{-1,0, \ldots, n\}$ with $\hat{G}(A)$ connected,

$$
|[A]|=2+\sum_{i \in A}\left(a_{i}-2\right),
$$

so removing indices with $a_{i}=2$ from $A$ does not change $|[A]|$. If $\left[A_{j}\right]$ is unbreakable, by Lemma $3.10 A_{j}$ has at most one element $i$ with $a_{i} \geq 3$, so $\left|\left[A_{j} \cap A_{k}\right]\right|$ is either |[ $\left.A_{j}\right] \mid$ if $A_{k}$ contains the index for which $a_{i} \geq 3$, or 2 if it does not.

Definition 4.15 Following Greene [10], say that two intervals $\left[A_{j}\right]$ and $\left[A_{k}\right]$ are distant if they do not intersect and $\left\langle\left[A_{j}\right],\left[A_{k}\right]\right\rangle=0$, that they are consecutive and write $A_{j} \dagger A_{k}$ if they do not intersect and $\left\langle\left[A_{j}\right],\left[A_{k}\right]\right\rangle=-1$, and that they share a common endpoint if they intersect and $\left\langle\left[A_{j}\right],\left[A_{k}\right]\right\rangle=\left|\left[A_{j} \cap A_{k}\right]\right|-1$. If $A_{j}$ and $A_{k}$ share a common endpoint and $A_{j} \subset A_{k}$, write $A_{j} \prec A_{k}$. Say that two intervals abut if they are either consecutive or share a common endpoint. Write $A_{j} \pitchfork A_{k}$ if $A_{j} \cap A_{k} \neq \varnothing$ and they do not share a common end point.

Definition 4.16 Let the intersection graph $G(T)$, where $T \subset S$ is a subset of the standard basis, be the graph with vertex set $T$, and with an edge between $v_{i}$ and $v_{j}$ (write $v_{i} \sim v_{j}$ ) whenever $\left[A_{i}\right]$ and $\left[A_{j}\right]$ abut. If $v_{i} \sim v_{j}$ and $i<j$, we say $v_{i}$ is a smaller neighbor of $v_{j}$.

For the intersection graph to be a useful concept, we need to somehow relate abutment of intervals to pairings in the lattice. First, we need to know how the pairings between intervals relate to the pairings between standard basis vectors:

Lemma 4.17 For any two standard basis vectors $v_{i}, v_{j}$ with $\{i, j\} \neq\{1, f\}$, we have $\left\langle v_{i}, v_{j}\right\rangle= \pm\left\langle\left[A_{i}\right],\left[A_{j}\right]\right\rangle$. Furthermore, if both $v_{i}$ and $v_{j}$ have norm at least 3 , then $\left\langle v_{i}, v_{j}\right\rangle=\epsilon_{i} \epsilon_{j}\left\langle\left[A_{i}\right],\left[A_{j}\right]\right\rangle$. 
Proof If $\epsilon_{i}=\epsilon_{j},\left\langle v_{i}, v_{j}\right\rangle=\left\langle\left[A_{i}\right],\left[A_{j}\right]\right\rangle$ since $\tau$ is a reflection. Otherwise,

$$
\left\langle\left[A_{i}\right],\left[A_{j}\right]\right\rangle=\left\langle\tau\left(v_{i}\right), v_{j}\right\rangle=\left\langle v_{i}, \tau\left(v_{j}\right)\right\rangle,
$$

so the desired results will hold as long as either $\tau\left(v_{i}\right)=-v_{i}$ or $\tau\left(v_{j}\right)=-v_{j}$. It follows from the proof of Proposition 4.13 that the only intervals (corresponding to standard basis vectors) that are not simply negated by $\tau$ are $x_{*}, x_{* *}$, intervals with left endpoint 0 and, if $a_{0}=2$, intervals with left endpoint 1 . Note that any interval with left endpoint 0 is not tight since the tight vector pairs differently with $x_{*}$ and $x_{* *}$.

First, consider the case $a_{0} \geq 3$. If one of $\left[A_{i}\right]$ or $\left[A_{j}\right]$ is either $x_{*}$ or $x_{* *}$ and the other is an interval with left end 0 (which in particular is neither tight nor $x_{*}$ or $x_{* *}$ ), then the result follows from the facts that $\tau\left(x_{*}\right)=x_{* *}$ and Lemma 4.11. If both $\left[A_{i}\right]$ and $\left[A_{j}\right]$ are intervals starting at 0 , then neither one is tight, by Lemma 4.5 both are unbreakable. Therefore, since $a_{0} \geq 3$ and 0 is in both $A_{i}$ and $A_{j}$, every other $k \in A_{i} \cup A_{j}$ has $a_{k}=2$; see Lemma 3.10. Assume $\left\langle v_{i}, v_{j}\right\rangle \neq \pm\left\langle\left[A_{i}\right],\left[A_{j}\right]\right\rangle$, then it follows from (14) that $\left|v_{i}\right|=\left|v_{j}\right|=a_{0}$. Note that

$$
\left\langle v_{i}, v_{j}\right\rangle=\left\langle\tau\left(\left[A_{i}\right]\right),\left[A_{j}\right]\right\rangle=\left\langle\left[A_{i}\right], \tau\left(\left[A_{j}\right]\right)\right\rangle
$$

and $\tau\left(\left[A_{i}\right]\right)=-\left[A_{i}\right]-x_{*}-x_{* *}$, and we can compute that $\left\langle v_{i}, v_{j}\right\rangle$ is either $3-a_{0}$ if $A_{i} \neq A_{j}$, or $2-a_{0}$ if $A_{i}=A_{j}$. We can check that neither of these can occur: for any two standard basis vectors, $\left\langle v_{i}, v_{j}\right\rangle \geq-1$, so $a_{0}$ is either 3 or 4 , and $\left\langle v_{i}, v_{j}\right\rangle$ is either 0 or -1 . By Lemma 4.11, $\left\langle v_{i}, v_{1}\right\rangle=\left\langle v_{j}, v_{1}\right\rangle=\left\langle v_{i}, v_{f}\right\rangle=\left\langle v_{j}, v_{f}\right\rangle=-1$, so $1 \in \operatorname{supp}^{+} v_{i} \cap \operatorname{supp}^{+} v_{j}$. Therefore, $\left\langle v_{i}, v_{j}\right\rangle=0$, and $a_{0}=3$. However, using Lemma 4.9 , the only possible standard basis vectors of norm 3 that have pairing -1 with both $v_{1}$ and $v_{f}$ are $e_{1}+e_{f-2}-e_{f-1}$ and $e_{1}+e_{f}-e_{f+1}$, but these have pairing 1 with each other. This contradiction shows that there cannot be two standard basis vectors corresponding to intervals with left endpoint 0 . Therefore, given any two standard basis vectors of norm at least 3 (so neither one is $x_{*}$ or $x_{* *}$ ), one of them must be negated by $\tau$, which proves the last statement in this case.

When $a_{0}=2$, the situation is similar. Using Proposition 4.13, the only standard basis intervals that are not negated by $\tau$ are $x_{*}, x_{* *}, x_{0}$ and intervals that start at 1 . The vector $x_{0}$ is fixed by $\tau$. For any interval $[A]$ with left end $1, \tau([A])=$ $-[A]-x_{*}-x_{* *}-2 x_{0}$, so $\left\langle x_{*},[A]\right\rangle=\left\langle x_{*}, \tau([A])\right\rangle=0$, and this also holds for $x_{* *}$. It only remains to rule out the case in which $\left[A_{i}\right]$ and $\left[A_{j}\right]$ are both intervals with left end 1 . In this case, again $v_{i}$ and $v_{j}$ are unbreakable, so 1 is the only index $k \in A_{i} \cup A_{j}$ with $a_{k} \geq 3,\left|v_{i}\right|=\left|v_{j}\right|=a_{1}$, and $\left\langle v_{i}, v_{j}\right\rangle$ is either $3-a_{1}$ or $2-a_{1}$, always $\leq 0$. 
However,

$$
\left\langle v_{i}, x_{*}\right\rangle=\left\langle v_{i}, x_{* *}\right\rangle=\left\langle v_{j}, x_{*}\right\rangle=\left\langle v_{j}, x_{* *}\right\rangle=0
$$

and

$$
\left\langle v_{i}, x_{0}\right\rangle=\left\langle v_{j}, x_{0}\right\rangle=-1 \text {, }
$$

so $\operatorname{supp}^{+}\left(v_{i}\right) \cap\{0,1,2,3\}=\operatorname{supp}^{+}\left(v_{j}\right) \cap\{0,1,2,3\}=\{2,3\}$. This forces $\left\langle v_{i}, v_{j}\right\rangle \geq 1$, a contradiction. Again, since $x_{*}, x_{* *}$, and $x_{0}$ all have norm 2, at least one of any pair of standard basis vectors of norm at least 3 is negated by $\tau$, and the conclusion follows.

Because all of the $A_{j}$ are subintervals of one interval and the pairings between the $\left[A_{j}\right]$ are, up to sign, the same as the pairings between standard basis vectors, many of the results from [10] about the intersection graph carry over unchanged to this situation. Most importantly, [10, Lemma 4.4] holds unchanged:

Lemma 4.18 [10, Lemma 4.4] If $v_{i}$ and $v_{j}$ are unbreakable standard basis vectors of norm at least 3 , then $\left|\left\langle v_{i}, v_{j}\right\rangle\right| \leq 1$, with equality if and only if $\left[A_{i}\right]$ and $\left[A_{j}\right]$ are consecutive and $\left\langle v_{i}, v_{j}\right\rangle=-\epsilon_{i} \epsilon_{j}$.

The proof of Lemma 4.18, which will not be repeated here, is identical to the one Greene gives. The overall strategy is similar to the one used in Lemma 4.17: showing that, for unbreakable standard basis vectors $v_{i}, v_{j}$ of norm at least 3 , if $A_{i} \cap A_{j}$ contains an index $k$ with $a_{k} \geq 3$ then the pairing $\left\langle v_{i}, v_{j}\right\rangle$ will be too large given the form of the standard basis vectors. The proof actually shows:

Corollary 4.19 If $v_{i}$ and $v_{j}$ are unbreakable standard basis vectors of norm at least 3, then $z_{i} \neq z_{j}$, where $z_{i}$ is as defined in Definition 3.11.

Corollary 4.20 If $\{i, j\} \neq\{1, f\}$ and $v_{i} \sim v_{j}$, then $\left\langle v_{i}, v_{j}\right\rangle \neq 0$. If $v_{i} \nsim v_{j}$ and $v_{i}$ and $v_{j}$ are both unbreakable, then $\left\langle v_{i}, v_{j}\right\rangle=0$.

Proof If $v_{i} \sim v_{j}$, then $\left\langle\left[A_{i}\right],\left[A_{j}\right]\right\rangle$ is equal to either -1 or $\left|\left[A_{i} \cap A_{j}\right]\right|-1 \neq 0$. It follows from Lemma 4.17 that $\left\langle v_{i}, v_{j}\right\rangle \neq 0$.

If $v_{i} \nsim v_{j}$, by Lemma 4.17 we only need to show $\left\langle\left[A_{i}\right],\left[A_{j}\right]\right\rangle=0$. If $A_{i}$ and $A_{j}$ are distant, we are done. If $A_{i} \pitchfork A_{j}$, by Lemma 4.14 and its proof

$$
\left\langle\left[A_{i}\right],\left[A_{j}\right]\right\rangle=\left|\left[A_{i} \cap A_{j}\right]\right|-2 .
$$

By Corollary 4.19, $A_{i} \cap A_{j}$ does not contain any vertex with norm $\geq 3$, so $\left|\left[A_{i} \cap A_{j}\right]\right|=2$ and our conclusion holds. 
In particular, when $S$ contains no breakable vectors, $G(S)$ is almost the same as $\widehat{G}(S)$, with the only differences being that $G(S)$ has an edge between $v_{1}$ and $v_{f}$ while $\widehat{G}(S)$ does not.

Corollary 4.21 At most one unbreakable vector other than $v_{1}$ or $v_{f}$ neighbors $v_{1}$ in $G(S)$. The same holds for $v_{f}$ and, when $a_{0}=2$, for $v_{2}$.

Proof If $v_{j} \sim v_{1}$ or $v_{j} \sim v_{f}$, then using Proposition 4.13 and Corollary 4.20 we get either $j \in\{1, f\}$ or $0 \in A_{j}$. If $a_{0} \geq 3$ and $v_{j}$ is unbreakable, then $z_{j}=0$, so no other unbreakable standard basis vector corresponds to an interval containing 0 . When $a_{0}=2$, by Proposition 4.13 and Corollary $4.20 v_{2}$ is the only neighbor of $v_{1}$ or $v_{3}$, and when $v_{j} \sim v_{2}$ for some $j \notin\{1, f\}$ and $v_{j}$ is unbreakable, $z_{j}=1$.

Once we have Lemma 4.18, almost all of the remaining results of [10, Section 4] carry over. In particular, we have versions of [10, Lemmas 4.8 and 4.10], with identical proofs.

Definition 4.22 A claw in a graph $G$ is a set of four vertices $(v ; x, y, z)$ with $v$ adjacent to $x, y$ and $z$, and no two of $x, y$ and $z$ adjacent to each other.

Lemma 4.23 [10, Lemma 4.8] $G(S)$ has no claws.

Let $\bar{S}$ be the set of unbreakable elements of $S$, and let $S_{g}=\left\{v_{1}, v_{2}, \ldots, v_{g}\right\}$.

Definition 4.24 A triple $\left(v_{i}, v_{j}, v_{k}\right) \in(\bar{S})^{3}$ is a heavy triple if each of $v_{i}, v_{j}$ and $v_{k}$ have norm at least 3 , and any two of them are connected by a path in $G(\bar{S})$ that does not pass through the third. If the heavy triple $\left(v_{i}, v_{j}, v_{k}\right)$ spans a triangle, we say it is a heavy triangle.

Lemma 4.25 [10, Lemma 4.10] No triple is heavy.

Proof Suppose that $\left(v_{i}, v_{j}, v_{k}\right)$ is heavy, with $z_{i}<z_{j}<z_{k}$. Then any path from $v_{i}$ to $v_{k}$ in $G(\bar{S})$ would contain an unbreakable interval containing $z_{j}$, contradicting Corollary 4.19 .

The last of Greene's results that we will need to characterize the D-type lattices that are isomorphic to changemaker lattices is a description of the cycles that can occur in $G(S)$. However, this will require more modification since Greene's proof relies on the intervals $\left[A_{i}\right]$ being linearly independent, which fails in this situation since $\left[A_{1}\right]=\left[A_{f}\right]$. Luckily, this is the only problem: 
Lemma 4.26 $\left[A_{2}\right],\left[A_{3}\right], \ldots,\left[A_{n+2}\right]$ and $\left[A_{n+3}\right]$ are linearly independent.

Proof We remind the reader we are using the notation that $L=(\sigma)^{\perp}$ is a changemaker lattice isomorphic to some D-type lattice; also that we identify $x_{*}, x_{* *}, x_{0}, \ldots, x_{n}$ with elements of $L$ according to an isomorphism chosen as in Lemma 4.8. Consider the map $\pi: L \otimes \mathbb{Q} \rightarrow L \otimes \mathbb{Q}$ given by $\pi(x)=x-\tau(x)$. For any $x \in L \otimes \mathbb{Q}, \pi(\tau(x))=-\pi(x)$, so in particular $\pi\left(\left[A_{i}\right]\right)= \pm \pi\left(v_{i}\right)$ for each standard basis vector $v_{i}$.

When $a_{0} \geq 3, \tau$ is the reflection in a line, so $\operatorname{im} \pi$ has dimension $n+2$. Since $\left(v_{1}, v_{2}, \ldots, v_{n+3}\right)$ spans $L \otimes \mathbb{Q}$, we get that $\left(\pi\left(v_{1}\right), \pi\left(v_{2}\right), \ldots, \pi\left(v_{n+3}\right)\right)$ spans im $\pi$. However, $\pi\left(v_{1}\right)=-\pi\left(v_{f}\right)$, so actually $\pi\left(v_{2}\right), \ldots, \pi\left(v_{n+3}\right)$ suffice. Since im $\pi$ has dimension $n+2$, this means that $\pi\left(v_{2}\right), \ldots, \pi\left(v_{n+3}\right)$ are linearly independent. Since $\pi\left(\left[A_{i}\right]\right)= \pm \pi\left(v_{i}\right)$ for each $i$, a linear dependence among $\left[A_{2}\right], \ldots,\left[A_{n+3}\right]$ would induce one among $\pi\left(v_{2}\right), \ldots, \pi\left(v_{n+3}\right)$, and the conclusion follows.

When $a_{0}=2, \tau$ is the reflection in a plane, so $\operatorname{im}(\pi)$ has dimension $n+1$, and a basis is $\left(\pi\left(v_{3}\right), \pi\left(v_{4}\right), \ldots, \pi\left(v_{n+3}\right)\right)$. The same argument as before gives that $\left[A_{3}\right],\left[A_{4}\right], \ldots,\left[A_{n+3}\right]$ are linearly independent. It remains only to be seen that $\left[A_{2}\right]=v_{2}$ is not in the span of $\left(\left[A_{3}\right], \ldots,\left[A_{n+3}\right]\right)$. Note that $\left[A_{3}\right]=v_{1}$ and, for $j>3$, $\tau\left(v_{j}\right)=-v_{j}$ or $-v_{1}-2 v_{2}-v_{3}-v_{j}$, so

$$
\operatorname{span}\left(\left[A_{3}\right], \ldots,\left[A_{n+3}\right]\right) \subset \operatorname{span}\left(v_{1}, v_{1}+2 v_{2}+v_{3}, v_{4}, \ldots, v_{n+3}\right),
$$

and the right side does not contain $v_{2}$.

Once we have this, a proof identical to that of Greene's Lemma 3.8 [10] gives:

Lemma 4.27 Any simple cycle in $G\left(\left\{v_{2}, v_{3}, \ldots, v_{n+3}\right\}\right)$ induces a complete subgraph.

Actually, since $\left[A_{1}\right]=\left[A_{f}\right]$, for any $j \notin\{1, f\}, v_{j} \sim v_{1}$ if and only if $v_{j} \sim v_{f}$, so the same statement holds for $G\left(\left\{v_{1}, v_{2}, \ldots, v_{f-1}, v_{f+1}, \ldots, v_{n+3}\right\}\right)$.

Lemma 4.28 For any simple cycle $C \subset G(S)$, one of the following holds:

- The vertex set $V(C)$ induces a complete subgraph of $G(S)$.

- $C$ has exactly four vertices, of which three are $v_{1}, v_{f}$ and $v_{f-1}$, and $v_{f-1}$ is breakable.

Proof If $C$ contains exactly 3 vertices, $C$ is a complete subgraph. So we assume $C$ contains at least 4 vertices. If either $v_{1}$ or $v_{f}$ is not in $C$, the previous lemma says that 
$C$ induces a complete subgraph. Otherwise, $v_{1}, v_{f} \in V(C)$. Since any neighbor (other than $v_{1}$ ) of $v_{f}$ also neighbors $v_{1}, v_{1}$ must have two neighbors $v_{j}$ and $v_{k}$ also in $C$, which will also neighbor $v_{f}$. By Corollary 4.21, one of these, say $v_{k}$, is breakable. By Lemmas 4.5 and $4.11, k=f-1$, and neither $v_{1}$ nor $v_{f}$ has any other neighbors. This means that the vertices of $C$ other than $v_{1}, v_{f}, v_{j}$ and $v_{f-1}$ all lie on a path $\gamma$ for which one end neighbors $v_{j}$ and the other neighbors $v_{f-1}$. It remains to see only that $\gamma$ is empty. Since $v_{f}$ neighbors both $v_{j}$ and $v_{f-1}$, if $\gamma$ were nonempty we could form a new simple cycle going from $v_{j}$ to $v_{f-1}$ along $\gamma$, then to $v_{f}$ and back to $v_{j}$. This does not contain $v_{1}$, so induces a complete subgraph. This means that any vertex of $\gamma$ neighbors $v_{f}$, so $\gamma$ is empty.

Lemma 4.29 Any cycle in $G(S)$ has three vertices, unless it contains a breakable vector, in which case it can contain 4 .

Proof By Corollary 4.21 and Lemma 4.28, we can assume that $V(C)$ does not contain both $v_{1}$ and $v_{f}$ and that $V(C)$ induces a complete subgraph. If $v_{f-1}$ is breakable and in $V(C)$, then $V(C) \backslash\left\{v_{f-1}\right\}$ will still induce a complete subgraph, so it suffices to assume that $V(C)$ contains no breakable vector. If $V(C)$ had more than two vectors of norm at least 3 , any three of them would form a heavy triple. Since $V(C)$ induces a complete subgraph, any two vectors of norm 2 in $V(C)$ are of the form $v_{i}=e_{i-1}-e_{i}$ and $v_{i+1}=e_{i}-e_{i+1}$ for some index $i$. However, any other standard basis vector $v_{j}$ that neighbored both $v_{i}$ and $v_{i+1}$ would have to have a gappy index at either $i-1$ or $i$, which cannot happen by Lemma 4.3 because $v_{i}$ and $v_{i+1}$ both have norm 2 . Therefore, $V(C)$ has at most one vector of norm 2, and at most two vectors of norm at least 3 , so has at most 3 vectors overall. Since $C$ is a cycle, it must have exactly 3 .

The main use we will have for this characterization of cycles is the following lemma that restricts the possible forms of gappy vectors, which is [10, Lemma 7.1]. As usual, the proof will not be repeated.

Lemma 4.30 [10, Lemma 7.1] Suppose that $v_{g} \in S$ is gappy, and that $S$ contains no breakable vector. Then $v_{g}$ is the unique gappy vector, $v_{g}=-e_{g}+e_{g-1}+\cdots+e_{j}+e_{k}$ for some $k+1<j<g$, and $v_{k}$ and $v_{k+1}$ belong to distinct components of $G\left(S_{g-1}\right)$.

\section{Blocked vectors}

In this section, we define blocked vectors and prove some lemmas about blocked vectors. These lemmas will be useful in the later sections. 
Definition 5.1 Let $m \in\{1,2, \ldots, n+2\}$. A vector $v_{i} \in S_{m}$ is $(m, N)$-blocked if $v_{i} \nsim v_{j}$ for any $m<j \leq N$. Otherwise, $v_{i}$ is $(m, N)$-open. When $N=n+3$ and $S_{N}=S$, we simply say $v_{i}$ is $m$-blocked or $m$-open.

Lemma 5.2 When $v_{f-1}$ is unbreakable, $v_{1}$ and $v_{f}$ are $f$-blocked.

Proof If $a_{0} \geq 3$, by Corollary $4.19 A_{f-1}$ is the unique unbreakable interval containing $x_{0}$, so $v_{1}, v_{f}$ are $f$-blocked. If $a_{0}=2$, then $f=3$ and $\left|v_{1}\right|=\left|v_{2}\right|=\left|v_{3}\right|=2$ by Lemma 4.8. Since 0,1,2 cannot be gappy indices, no vector other than $v_{2}$ can neighbor $v_{1}$ and $v_{3}$ at the same time, so $v_{1}$ and $v_{3}$ are 3 -blocked by Lemma 4.11.

Below, we introduce the notion of an $(m, N)$-blocking neighbor. We will see in Lemma 5.5 that a standard basis vector is $(m, N)$-blocked if it has two $(m, N)-$ blocking neighbors that do not abut each other.

Definition 5.3 Let $v_{i}, v_{j} \in S_{m}$ be such that $v_{j} \sim v_{i}$. We say $v_{j}$ is an $(m, N)$-blocking neighbor of $v_{i}$ if one of the following holds:

(1) $v_{j}$ is $(m, N)$-blocked.

(2) $\left|v_{i}\right| \geq 3,\left|v_{j}\right| \geq 3$, both unbreakable; $v_{\ell}$ is unbreakable for $m<\ell \leq N$.

(3) $j=i \pm 1$ and $\left|v_{i}\right|=\left|v_{j}\right|=2$.

(4) $j=i \pm 1,\left|v_{\max (i, j)}\right|=2$ and $v_{\min (i, j)}$ is unbreakable; $v_{\ell}$ is $(m, N)$-blocked for $\ell<\min (i, j)$ and $v_{\ell}$ is unbreakable for $m<\ell \leq N$.

When $N=n+3$ and $S_{N}=S$, we simply say $v_{j}$ is an $m$-blocking neighbor of $v_{i}$.

Lemma 5.4 Suppose $v_{j}$ is an $(m, N)$-blocking neighbor of $v_{i}$. If $v_{i} \sim v_{k}$ for some $m<k \leq N$, then $v_{j} \nsim v_{k}$.

Proof We prove this case by case.

(1) Since $v_{j}$ is $(m, N)$-blocked, by definition $v_{k} \nsim v_{j}$.

(2) For contradiction, suppose $v_{k} \sim v_{j}$. Since $v_{k} \sim v_{i}$ and $v_{k} \sim v_{j},\left|v_{k}\right| \geq 3$. Otherwise, if $\left|v_{k}\right|=2, v_{k}$ has only one smaller neighbor. Since we assume $v_{i}, v_{j}$ and $v_{k}$ are unbreakable, $\left(v_{i}, v_{j}, v_{k}\right)$ is a heavy triangle; see Lemma 4.25.

(3) Suppose $v_{k} \sim v_{j}$ for contradiction. Assume $j=i+1$. This assumption is made without loss of generality because the situation $v_{k} \sim v_{i}, v_{j}$ and $v_{i} \sim v_{j}$ is symmetric 
in $i$ and $j$. Since $v_{k} \sim v_{j}$ and $v_{j}=e_{i}-e_{i+1}(i>0)$, exactly one of $i$ and $i+1$ is in $\operatorname{supp} v_{k}$. Since $\left|v_{j}\right|=2, j-1=i$ cannot be a gappy index by Lemma 4.3. Therefore, $i \notin \operatorname{supp} v_{k}$ and $i+1 \in \operatorname{supp} v_{k}$. Since $v_{k} \sim v_{i}$ and $v_{i}=e_{i-1}-e_{i}$, we get $i-1 \in \operatorname{supp} v_{k}$. This contradicts Lemma 4.3 since $\left|v_{i}\right|=2$.

(4) Again, suppose $v_{k} \sim v_{j}$ for contradiction. Assume $j=i+1$ without loss of generality, since the situation is symmetric in $i$ and $j$. From item (3), we may assume $\left|v_{i}\right| \geq 3$ and $\left|v_{j}\right|=2$. As in item (3), $i \notin \operatorname{supp} v_{k}$ and $i+1 \in \operatorname{supp} v_{k}$. Let $\ell=\min \operatorname{supp} v_{k}$. Since $v_{k} \sim v_{i}$ and $i \notin \operatorname{supp} v_{k}$, we get $\ell<i$.

If $\ell=0$, let $s$ be the first gappy index of $v_{k}$; then $s<i$, and we claim that $v_{k} \sim v_{s+1}$. Since $\left\langle v_{k}, v_{s+1}\right\rangle>0$, if $v_{k} \nsim v_{s+1}$, it follows from Lemmas 4.14 and 4.17 and Corollary 4.20 that $v_{s+1}$ is breakable, $A_{k} \pitchfork A_{s+1}$ and $\epsilon_{k}=\epsilon_{s+1}$. Thus, up to applying $\tau, v_{k}-v_{s+1}$ becomes $\left[A_{k}\right]-\left[A_{s+1}\right]$, which is reducible since it is the signed sum of two distant intervals, a contradiction to Lemma 4.7. Since $v_{k} \sim v_{s+1}$ and $s+1 \leq i$, we have $s=i-1$ by our assumption. Since $\left|v_{i}\right| \geq 3$, we must have $\left\langle v_{i}, v_{k}\right\rangle \geq 2$. However, since $v_{i}$ and $v_{k}$ are both unbreakable, this contradicts Lemma 4.18.

If $\ell>0$, then $\left\langle v_{\ell}, v_{k}\right\rangle=-1$. Since $v_{k} \nsim v_{\ell}$, it follows from Corollary 4.20 that $v_{\ell}$ is breakable, so $\ell=f-1$. By Lemmas 4.17 and 4.14, $\left|v_{k}\right|=3$. Using Lemma 4.9, we get $v_{k}=-e_{k}+e_{k-1}+e_{\ell}$. Since $\ell<i<m<k, k>\ell+2$ so $k-1>\ell+1$. Since $v_{\ell+1}=v_{f}=-e_{\ell+1}+e_{\ell}$, we have $\left\langle v_{k}, v_{\ell+1}\right\rangle=1$, so $v_{k} \sim v_{\ell+1}$. If $\ell+1<i$, then $v_{\ell+1}$ is $m$-blocked by assumption, a contradiction. If $\ell+1=i,\left|v_{i}\right|=2$, a contradiction to our assumption that $\left|v_{i}\right| \geq 3$.

Lemma 5.5 If $v_{i} \in S_{m}$ has two $(m, N)$-blocking neighbors $v_{j_{1}}, v_{j_{2}} \in S_{m}$ such that $v_{j_{1}} \nsim v_{j_{2}}$, then $v_{i}$ is $(m, N)$-blocked.

Proof Suppose $v_{k} \sim v_{i}$ for some $m<k \leq N$. By Lemma 5.4, $v_{j_{1}} \nsim v_{k}$ and $v_{j_{2}} \nsim v_{k}$. However, this would imply $\left\{v_{i} ; v_{j_{1}}, v_{j_{2}}, v_{k}\right\}$ is a claw.

Lemma 5.6 Suppose that $v_{k}$ is just right for all $k>m$, and that $v_{j} \in S_{m}$ is unbreakable. Then $v_{i} \in S_{m}$ is $m$-blocked if either of the following holds:

(1) $\operatorname{supp} v_{j}$ has at least 4 elements $\geq i$.

(2) $\operatorname{supp} v_{j}$ has at least 3 elements $\geq i$, and $v_{j}$ is $m$-blocked.

Proof In the first case, any $v_{k}$ neighboring $v_{i}$ with $k>m$ would have min supp $v_{k} \leq i$, hence $\left|\operatorname{supp} v_{k} \cap \operatorname{supp} v_{j}\right| \geq 4$ and $\left\langle v_{k}, v_{j}\right\rangle \geq 2$, contradicting Lemma 4.18. In the second case if $v_{i} \sim v_{k}$ for some $k>m,\left\langle v_{k}, v_{j}\right\rangle \geq 1$, so $v_{k} \sim v_{j}$, a contradiction. 
Lemma 5.7 Suppose $v_{i}$ is $(m, N)$-blocked for all $i<m$. If $S_{m-1}$ contains at least one unbreakable vector of norm $\geq 3$, then $\left|v_{j}\right|=2$ for all $m<j \leq N$.

Proof We induct on $N-m$. The base case $N-m=0$ is trivial. Inductively, suppose the lemma holds whenever $N-m \leq k$ for any nonnegative integer $k$. The following shows that the lemma also holds when $N-m=k+1$.

Since $v_{i}$ is $(m, N)$-blocked for all $i<m$, either min supp $v_{m+1}=0$ or $\left|v_{m+1}\right|=2$. Since $S_{m-1}$ contains some unbreakable vector $v_{i}$ of norm $\geq 3, \min \operatorname{supp} v_{m+1}=0$ would imply $v_{m+1} \sim v_{i}$, but $v_{i}$ is $m$-blocked. Therefore, $\left|v_{m+1}\right|=2$.

Since $S_{m-1}$ contains a high norm vector, $v_{m}$ has some smaller neighbor $v_{s}$, which is $(m, N)$-blocked by assumption. By Definition 5.3(1) and (4), $v_{s}$ and $v_{m+1}$ are $(m+1, N)$-blocking neighbors of $v_{m}$. Since $\left|v_{m+1}\right|=2$ and $s<m, v_{s} \nsim v_{m+1}$. Hence, by Lemma 5.5, $v_{m}$ is $(m+1)$-blocked.

Since $N-(m+1) \leq k$ and $v_{i}$ is $(m+1, N)$-blocked for all $i<m+1$, the inductive assumption implies $\left|v_{j}\right|=2$ for all $m+1<j \leq N$. Since we have also shown $\left|v_{m+1}\right|=2$, this finishes the induction proof.

Lemma 5.8 If $G\left(S_{m}\right)$ is disconnected, every connected component has at least one $m$-open vector.

Proof Otherwise, a component of $G\left(S_{m}\right)$ would still be a component in $G\left(S_{n+3}\right)$, disconnected from the rest of $G\left(S_{n+3}\right)$. However, this contradicts Corollary 3.3, which says the lattice is indecomposable and $G\left(S_{n+3}\right)$ is connected.

Lemma 5.9 Suppose $S$ contains no breakable vector. If $S_{m}$ contains at least one vector of norm $\geq 3$, and contains exactly one $m$-open vector $v_{j}$ with $j<m$, then $v_{m+1}$ neighbors $v_{j}$ and has no other smaller neighbors, and $\left|v_{k}\right|=2$ for all $k>m+1$.

Proof Since $S_{m}$ has a high norm vector, $v_{m+1}$ has some smaller neighbor, which must be $v_{j}$. By Lemma 5.8, $G\left(S_{m}\right)$ is connected, so $v_{j}$ neighbors some $v_{i} \in S_{m}$. Since $v_{j}$ is the only $m$-open vector in $S_{m}, v_{i}$ is $m$-blocked, and $v_{i}$ is an $m$-blocking neighbor of $v_{j}$ by Definition 5.3(1). Since $v_{m+1} \sim v_{j}$ and $j<m,\left|v_{m+1}\right| \geq 3$. By Definition 5.3(2), $v_{m+1}$ is an $(m+1)$-blocking neighbor of $v_{j}$. By Lemma 5.5, $v_{j}$ is $(m+1)-$ blocked. The rest follows by Lemma 5.7. 


\section{$6 \quad a_{0}=2$}

In this section, we assume $a_{0}=2$. By Lemma 4.8, $f=3$ and $v_{2}=e_{1}-e_{2}$.

Lemma 6.1 If $v_{s} \sim v_{2}$ for $s>3$, then $v_{2}$ is $s$-blocked.

Proof By Lemmas 4.8 and 4.10, $v_{2}=x_{0}$ and $\left|x_{1}\right|=a_{1} \geq 3$. If $v_{s} \sim v_{2}$, then, by Corollary $4.19, A_{s}$ is the unique interval containing $x_{1}$. Therefore, $v_{1}, v_{3}$ and $v_{s}$ are the only neighbors of $v_{2}$.

Lemma 6.2 The vector $v_{4}$ is just right and $\left|v_{4}\right| \in\{3,5\}$.

Proof By Lemma 5.2, $v_{1}$ and $v_{3}$ are 3-blocked. We can then use Lemmas 4.3 and 4.9 to get our conclusion.

Lemma 6.3 Suppose $\left|v_{4}\right|=3$. Unless $4=n+3$, we have $\left|v_{5}\right| \in\{2,6\}$ and $\left|v_{j}\right|=2$ for $j>5$.

Proof By Lemma 5.2, $v_{1}$ and $v_{3}$ are 3-blocked. Lemma 6.1 implies that $v_{2}$ is 4-blocked since $v_{4} \sim v_{2}$. If $v_{5}$ exists, then to avoid pairing with $v_{1}, v_{2}$ and $v_{3}$, either $\left|v_{5}\right|=2$ or $\left|v_{5}\right|=6$. In either case, $v_{4}$ is 5 -blocked by Lemma 5.5 and Definition 5.3(1) and (4) or (1)-(2). By Lemma 5.7, $\left|v_{j}\right|=2$ for all $j>5$.

Lemma 6.4 Suppose $\left|v_{4}\right|=5$. There is an index $s$ for which $v_{s}$ is just right, $\left|v_{s}\right|=$ $s-1$, and $\left|v_{j}\right|=2$ for $4<j<s$. Either $s=n+3$, or $\left|v_{s+1}\right|=3$ and $\left|v_{j}\right|=2$ for $j>s+1$.

Proof Note that $G(S)$ is connected while $G\left(S_{4}\right)$ has two components with vertex sets $\left\{v_{1}, v_{2}, v_{3}\right\}$ and $\left\{v_{4}\right\}$. Since $v_{1}$ and $v_{3}$ are 4-blocked, $v_{2}$ must be 4-open. Let $s>4$ be the (unique) index with $v_{s} \sim v_{2}$. Since $\left|v_{2}\right|=2,1$ cannot be a gappy index for $v_{s}$. Hence, $v_{s} \sim v_{2}$ implies $\{1,2\} \cap \operatorname{supp} v_{s}=\{2\}$, which then implies $3 \in \operatorname{supp} v_{s}$. To avoid having pairing 2 with $v_{4}$ (which would contradict Lemma 4.18), $4 \in \operatorname{supp} v_{s}$ and $v_{s} \sim v_{4}$.

For contradiction, suppose there exists $\left|v_{j}\right| \geq 3$ with $4<j<s$ chosen minimally. Since $\left|v_{5}\right|=\cdots=\left|v_{j-1}\right|=2$, we have $v_{4} \sim v_{5} \sim \cdots \sim v_{j-1}$. Since $v_{j}$ does not neighbor $v_{1}, v_{2}, v_{3}$, and $\left|\left\langle v_{4}, v_{j}\right\rangle\right| \leq 1$ by Lemma 4.18, we must have min supp $v_{j} \geq 4$. Thus, $v_{j}$ pairs nontrivially with one of $v_{4}, v_{5}, \ldots, v_{j-1}$. In other words, in $G\left(S_{S-1}\right)$, 
$v_{j}$ is in the same connected component as $v_{4}$. Since $\left|v_{i}\right|=2$ for $4<i<j$, no $i$ can be a gappy index for $4 \leq i<j-1$. Since $2,3,4 \in \operatorname{supp} v_{s},\{2,3, \ldots, j-1\} \subset \operatorname{supp} v_{s}$. Hence, $\left\langle v_{s}, v_{j}\right\rangle \geq\left|v_{j}\right|-2$, which in turn implies $v_{s} \sim v_{j}$, creating a heavy triple $\left(v_{4}, v_{j}, v_{s}\right)$ and resulting in a contradiction.

Therefore, $\left|v_{j}\right|=2$ for $4<j<s$. It follows from Lemma 4.3 that $v_{s}$ is just right, and $\left|v_{s}\right|=s-1$. So all the adjacency relations in $G\left(S_{S}\right)$ are

$$
v_{1} \sim v_{2}, \quad v_{1} \sim v_{3}, \quad v_{2} \sim v_{3}, \quad v_{2} \sim v_{s} \sim v_{4} \sim v_{5} \sim \cdots \sim v_{s-1} .
$$

By Lemma 6.1, since $v_{2} \sim v_{s}, v_{2}$ is $s$-blocked. By Lemma 5.5 and Definition 5.3(3) and (4), $v_{j}$ is $s$-blocked when $4<j<s-1$. By Lemma 5.5 and Definition 5.3(1) and (2), $v_{4}$ is $s$-blocked. By Lemma 5.5 and Definition 5.3(1), $v_{s}$ is $s$-blocked. So $v_{s-1}$ is the only possible $s$-open vector. If $s<n+3$, the connectivity of $G(S)$ implies that $v_{s-1}$ is $s$-open, so we can use Lemma 5.9 to conclude that $v_{s-1}$ is the only smaller neighbor of $v_{s+1}$, which implies that $v_{s+1}=e_{s-1}+e_{s}-e_{s+1}$, and $\left|v_{j}\right|=2$ for $j>s+1$.

To summarize, we have the following proposition:

Proposition 6.5 When $a_{0}=2$, one of the following holds:

(1) $\left|v_{4}\right|=3,\left|v_{i}\right|=2$ for $i \geq 5$.

(2) $\left|v_{4}\right|=3,\left|v_{5}\right|=6$, and $\left|v_{i}\right|=2$ for $i>5$.

(3) $\left|v_{4}\right|=5,\left|v_{i}\right|=2$ for $5 \leq i \leq s-1,\left|v_{s}\right|=s-1$, and $s=n+3$.

(4) $\left|v_{4}\right|=5,\left|v_{i}\right|=2$ for $5 \leq i \leq s-1,\left|v_{s}\right|=s-1,\left|v_{s+1}\right|=3$, and $\left|v_{j}\right|=2$ for $j \geq s+2$.

The corresponding changemakers are

- $\left(1,1,1,1,2^{[s]}\right)$ with $s>0$,

- $\left(1,1,1,1,4^{[s]}, 4 s+2,(4 s+6)^{[t]}\right)$,

where $a^{[s]}$ means $s$ copies of $a$.

Remark 6.6 We obtain the corresponding changemaker vectors by using Definition 4.1. Here, we do one example in detail for clarification: Proposition 6.5(1). We remind the reader that $\sigma_{0}=\sigma_{1}=1$; see equation (13). In the beginning of this section we obtained that $v_{2}=e_{1}-e_{2}$, that is, $\sigma_{2}=\sigma_{1}=1$; also that $f=3$, which in turn implies 
that $\left|v_{3}\right|=2$ and therefore $v_{3}=e_{2}-e_{3}$. That is, $\sigma_{3}=\sigma_{2}=1$. In item (1), $\left|v_{4}\right|=3$, which implies that $v_{4}=e_{2}+e_{3}-e_{4}$. This gives us that $\sigma_{4}=\sigma_{3}+\sigma_{2}=2$. We also have $\left|v_{i}\right|=2$ for $i \geq 5$. That is, $v_{i}=e_{i-1}-e_{i}$ and so $\sigma_{i}=2$ for $i \geq 5$. Therefore, $\sigma=\left(1,1,1,1,2^{[s]}\right)$, and that $s>0$ since the index 4 exists.

Remark 6.7 We combine degenerate cases when listing changemakers at the end of each section, and in our parametrization $s, t \geq 0$ unless otherwise specified. The parameter $s$ is not the previous $s$ in this section. The same convention is also used in later sections.

\section{Classification of $S_{f}$}

From now on, we assume $a_{0} \geq 3$. In this section, we classify the possible forms of $v_{1}, \ldots, v_{f}$. Always, $v_{1}=e_{0}-e_{1}$, and $v_{f}=e_{f-1}-e_{f}$.

Lemma 7.1 For $1<i<f-1$, we have $v_{i} \nsim v_{1}$. Moreover, $v_{f-1} \sim v_{1}$.

Proof By Lemma 4.11, $\left\langle v_{i}, v_{1}\right\rangle=\left\langle v_{i}, v_{f}\right\rangle=0$ for $1<i<f-1$. By Lemma 3.8, $\left\langle v_{f-1}, v_{1}\right\rangle \equiv\left\langle v_{f-1}, v_{f}\right\rangle=-1(\bmod 2)$, so $v_{f-1} \sim v_{1}$.

Proposition 7.2 When $f=3, v_{2}=2 e_{0}+e_{1}-e_{2}$.

Proof It follows from Lemma 7.1 that $v_{2} \sim v_{1}$, so $v_{2}=e_{1}-e_{2}$ or $v_{2}=2 e_{0}+e_{1}-e_{2}$. Since $\left\langle v_{2}, v_{1}\right\rangle \neq 0$, we have $x_{0} \in A_{2}$, so $\left|v_{2}\right| \geq\left|x_{0}\right|=a_{0} \geq 3$.

Proposition 7.3 When $f>3$, we have $v_{2}=e_{0}+e_{1}-e_{2}$, and $v_{j}$ is just right for all $j<f-1$.

(1) If $v_{f-1}$ is tight, then $\left|v_{j}\right|=2$ for $2<j<f-1$.

(2) If $v_{f-1}$ is just right, then $v_{f-1}=e_{1}+\cdots+e_{f-2}-e_{f-1}$, and one of the following holds:

(a) $\left|v_{j}\right|=2$ for $2<j<f-1$.

(b) $f=5$ and $\left|v_{3}\right|=4$.

(c) $f=6,\left|v_{3}\right|=2$ and $\left|v_{4}\right|=3$.

(3) If $v_{f-1}$ is gappy, then $f \geq 5$ and $v_{f-1}=e_{1}+e_{3}+\cdots+e_{f-2}-e_{f-1}$, and one of the following holds: 
(a) $\left|v_{j}\right|=2$ for $2<j<f-1$.

(b) $\left|v_{3}\right|=4$, and $\left|v_{j}\right|=2$ for $3<j<f-1$.

(c) $\left|v_{3}\right|=2,\left|v_{4}\right|=3$ and $\left|v_{j}\right|=2$ for $4<j<f-1$.

Proof By Lemma 7.1, $v_{2} \nsim v_{1}$, so $v_{2}=e_{0}+e_{1}-e_{2}$. Again using Lemma 7.1, to avoid a claw, any two smaller neighbors of $v_{f-1}$ other than $v_{1}$ neighbor each other. Therefore, to avoid a cycle of length 4 , which would violate Lemma $4.29, v_{f-1}$ has at most two smaller neighbors other than $v_{1}$. Furthermore, if $v_{f-1}$ is not tight and has two smaller neighbors, then one of them must have norm 2 , otherwise these three vectors would form a heavy triple. (Recall that $v_{f-1}$ is the only possible tight or breakable vector by Lemma 4.5 and Lemma 4.11.)

When there exists $\left|v_{j}\right|>2$ for some $2<j<f-1$, define

$$
s=\min \left\{2<j<f-1|| v_{j} \mid \geq 3\right\} .
$$

The only possible gappy index for $v_{s}$ is 1 , which cannot occur since then either $\left\langle v_{s}, v_{1}\right\rangle=-1$ or $\left\langle v_{s}, v_{2}\right\rangle=2$, contradicting Lemma 7.1 or Lemma 4.18. Thus, $v_{s}$ is just right. Let

$$
k=\min \operatorname{supp} v_{s} \leq s-2 \text {. }
$$

To avoid a claw $\left(v_{k} ; v_{k-1}, v_{k+1}, v_{s}\right)$, we must have $k \leq 2$. Since $\left\langle v_{s}, v_{1}\right\rangle=0$,

$$
k \in\{0,2\} .
$$

So

$$
\left\langle v_{s}, v_{2}\right\rangle= \pm 1 \neq 0
$$

Suppose $v_{f-1}$ is tight; then $\left\langle v_{f-1}, v_{2}\right\rangle=2=\left|v_{2}\right|-1$. It follows from Lemmas 4.14 and 4.17 that $\epsilon_{2}=\epsilon_{f-1}$, and $A_{2}$ shares an end with $A_{f-1}$. Since the left endpoint of $A_{f-1}$ is $v_{*}$ (see Proposition 4.13 and the paragraph following it), $A_{2}$ and $A_{f-1}$ share right endpoints. By Lemma 4.18 and (15), $A_{s} \dagger A_{2}$. Therefore, either $A_{s} \dagger A_{f-1}$ and $\left\langle v_{s}, v_{f-1}\right\rangle= \pm 1$, or $A_{s} \pitchfork A_{f-1}$ and $\left\langle v_{s}, v_{f-1}\right\rangle=\left|v_{s}\right|-2$. If $k=0$, then $\left\langle v_{s}, v_{f-1}\right\rangle=$ $\left|v_{s}\right|-1$, which does not fall into either case. Hence, $k=2$ and $\left\langle v_{s}, v_{f-1}\right\rangle=\left|v_{s}\right|-2$. We claim that $A_{s}$ and $A_{f-1}$ are not consecutive. Otherwise, $\left\langle v_{s}, v_{f-1}\right\rangle=1$ and $\epsilon_{s}=-\epsilon_{f-1}=-\epsilon_{2}$. Hence $\left\langle v_{s}, v_{2}\right\rangle=-\left\langle\left[A_{s}\right],\left[A_{2}\right]\right\rangle=1$, contradicting the fact that $k=2$. So $A_{s} \pitchfork A_{f-1}$. However, since $v_{s} \nsim v_{f-1},\left(v_{2} ; v_{s}, v_{f-1}, v_{3}\right)$ is a claw. Thus, $v_{s}$ cannot exist when $v_{f-1}$ is tight.

Suppose $v_{f-1}$ is just right. From the first paragraph we know that $v_{f-1}$ has at most one smaller neighbor with norm $\geq 3$. Since $v_{f-1} \sim v_{1}$, min supp $v_{f-1}=1$. Therefore, 
$v_{f-1} \sim v_{j}$ for $2<j<f-1$ if and only if $\left|v_{j}\right| \geq 3$. This implies $v_{1}$ and $v_{s}$ are the only smaller neighbors of $v_{f-1}$, and $\left|v_{i}\right|=2$ for $s<i<f-1$. Since $\left\langle v_{f-1}, v_{s}\right\rangle \leq 1$, either $s=3$ and $v_{s}=e_{0}+e_{1}+e_{2}-e_{3}$, or $s=4$ and $v_{s}=e_{2}+e_{3}-e_{4}$. In either case, unless $s+1=f-1,\left(v_{s} ; v_{2}, v_{s+1}, v_{f-1}\right)$ forms a claw. Thus, $f=s+2$.

Suppose $v_{f-1}$ is gappy. By Lemmas 4.5 and 4.11, $S$ contains no breakable vector. Since $v_{f-1} \sim v_{1}, 1=\min \operatorname{supp} v_{f-1}$. By Lemma 4.30,

$$
v_{f-1}=e_{1}+e_{j}+\cdots+e_{f-2}-e_{f-1}
$$

for some $j \geq 3$, and all other standard basis vectors are just right. By Lemma 4.9, $f-2 \in \operatorname{supp} v_{f-1}$, so $f-2 \geq j \geq 3$. Since $v_{f-1} \sim v_{2}$, then, by the discussion in the first paragraph of this proof,

$$
v_{i} \sim v_{f-1} \quad \text { and } \quad 2<i<f-1 \quad \Longrightarrow \quad\left|v_{i}\right|=2 .
$$

In particular, $v_{s} \nsim v_{f-1}$. If $\left|v_{i}\right|=2$ for any $2<i<f-1$, to avoid a claw $\left(v_{j} ; v_{j-1}, v_{j+1}, v_{f-1}\right)$ we must have either $j=3$ or $j=f-2$. If $j=f-2>3$, we would have a cycle

$$
v_{2} \sim v_{3} \sim \cdots \sim v_{f-1} \sim v_{2}
$$

of length $f-2>3$. So $j=3$ if $v_{s}$ does not exist. Now assume $v_{s}$ exists. Note $v_{s} \sim v_{2}$ by (15), $v_{f-1} \sim v_{2}$, and $v_{3} \sim v_{2}$ if $3<s$. To avoid a claw $\left(v_{2} ; v_{3}, v_{s}, v_{f-1}\right)$, either $s=3$ or $v_{f-1} \sim v_{3}$. In the first case, $v_{3}=e_{0}+e_{1}+e_{2}-e_{3}$. Since $\left|v_{3}\right|>2$, $v_{f-1} \nsim v_{3}$, forcing $j=3$. If $\left|v_{i}\right| \geq 3$ for some $i$ with $3<i<f-1$, by (16) we have $v_{i} \nsim v_{f-1}$. Hence $i=4$ and $v_{4}=e_{2}+e_{3}-e_{4}$, which would create a claw $\left(v_{2} ; v_{3}, v_{4}, v_{f-1}\right)$. Therefore, $\left|v_{i}\right|=2$ for all $3<i<f-1$. In the second case, by (16) we have $\left|v_{3}\right|=2$. Since $v_{f-1} \sim v_{3}, j=3$. Since $v_{s} \nsim v_{f-1}$, we have $s=4$ and $v_{s}=e_{2}+e_{3}-e_{4}$. If $\left|v_{l}\right|>2$ for some $l$ with $4<l<f-1$, we would have $\left\langle v_{l}, v_{f-1}\right\rangle>0$, contradicting (16).

\section{$8 f>3, v_{f-1}$ gappy}

Recall from Proposition 7.3 that when $f>3$ and $v_{f-1}$ is gappy, we have $f \geq 5$ and

$$
v_{2}=e_{0}+e_{1}-e_{2}, \quad v_{f-1}=e_{1}+e_{3}+\cdots+e_{f-2}-e_{f-1} .
$$

Proposition 8.1 When $v_{f-1}$ is gappy, one of the following holds:

(1) $f=n+3$. 
(2) $f=5,\left|v_{3}\right|=2,\left|v_{6}\right|=5$, and $\left|v_{j}\right|=2$ for $j>f+1$.

(3) $\left|v_{f+1}\right|=4$, and $\left|v_{j}\right|=2$ for $j>f+1$. Either $\left|v_{4}\right|=3$, or $\left|v_{3}\right|=4$.

Proof When $f=n+3$, we get item (1). If $f<n+3$, however, as before we will find it convenient to study the $f$-blocked vectors. First note that, by Lemmas 4.5 and 4.11, $S$ contains no breakable vector. Since $v_{f-1}$ is unbreakable, $v_{1}$ and $v_{f}$ are $f$-blocked by Lemma 5.2. Since $v_{f-1} \sim v_{1}$ and $v_{2}, v_{f-1}$ is also $f$-blocked by Lemma 5.5 and Definition 5.3(1)-(2). Lemma 4.30 then implies that $v_{j}$ is just right for $j>f$.

We argue that when either $f=5$ and $\left|v_{f-2}\right|=4$ or when $f>5$, the only $f$-open vector is $v_{f-2}$. When $f>5, v_{i}$ is $f$-blocked for $i<f-2$ by Lemma 5.6, (the $j$ in the statement of Lemma 5.6 is $f-1)$ and the only possible $f$-open vector is $v_{f-2}$. When $f=5$ and $\left|v_{3}\right|=4$,

$$
v_{3}=e_{0}+e_{1}+e_{2}-e_{3}, \quad v_{4}=e_{1}+e_{3}-e_{4} .
$$

Since $v_{3} \sim v_{2} \sim v_{4}$ and $v_{3} \nsim v_{4}$, we have that $v_{2}$ is $f$-blocked by Lemma 5.5 and Definition 5.3(1)-(2), and again the only possible $f$-open vector is $v_{f-2}$. This justifies the claim.

In the two cases (when $f=5$ and $\left|v_{f-2}\right|=4$ or when $f>5$ ), by Lemma 5.9 $v_{f+1} \sim v_{f-2}$, and $\left|v_{j}\right|=2$ for $j>f+1$. Since $v_{f-2}$ is the only smaller neighbor of $v_{f+1}$ and $v_{f+1}$ is just right, $\left|v_{f+1}\right|=4$. This will give us a subset of the cases in the first statement in item (3). (The item also includes the case $f=5$ and $\left|v_{f-2}\right|=2$, which will be obtained in the last paragraph; see below.) To see the second statement in item (3), note that when $\left|v_{3}\right|=\cdots=\left|v_{f-2}\right|=2$ and $\left|v_{f+1}\right|=4,\left(v_{f-1}, v_{2}, v_{f+1}\right)$ forms a heavy triple, so either case (3b) or case (3c) in Proposition 7.3 happens.

When $f=5$ and $\left|v_{3}\right|=2$, both $v_{2}$ and $v_{3}$ are possibly $f$-open. Since $v_{6} \nsim v_{1}, v_{4}$ or $v_{5}$, we have $\left|v_{6}\right| \in\{4,5\}$. In either case, $v_{2}$ and $v_{3}$ are 6-blocked by Lemma 5.6, and by Lemma $5.7\left|v_{j}\right|=2$ for $j>f+1$. In the case $\left|v_{6}\right|=5$, this gives us item (2). In the case $\left|v_{6}\right|=4$, we would have a heavy triple $\left(v_{2}, v_{4}, v_{6}\right)$, contradicting Lemma 4.25.

The corresponding changemakers are

- $\left(1,1,2,2,2^{[s]}, 2 s+3,2 s+3\right)$,

- $\left(1,1,2,4,4^{[s]}, 4 s+5,4 s+5,(8 s+14)^{[t]}\right)$,

- $\left(1,1,2,2,4^{[s]}, 4 s+3,4 s+3,(8 s+10)^{[t]}\right)$. 


\section{$9 f>3, v_{f-1}$ just right}

Assume $f>3$ and $v_{f-1}$ is just right. Recall from Proposition 7.3 that

$$
v_{2}=e_{0}+e_{1}-e_{2}, \quad v_{f-1}=e_{1}+\cdots+e_{f-2}-e_{f-1} .
$$

First, we consider cases (2b) and (2c) in Proposition 7.3.

Lemma 9.1 If there exists $\left|v_{j}\right| \geq 3$ for some $2<j<f-1$, then $f=n+3$.

Recall that in this case $f=j+2=5$ or 6 by Proposition 7.3(2b)-(2c).

Proof of Lemma 9.1 By Lemma 5.2, $v_{1}$ and $v_{f}$ are $f$-blocked, since $v_{f-1}$ is unbreakable. Therefore, $v_{f-1}$ is $f$-blocked by Lemma 5.5, since it neighbors $v_{1}$ and $v_{j}$. Because $G\left(S_{f}\right)$ is connected, any $G\left(S_{g}\right)$ is also connected for $g>f$. So there are no gappy vectors by Lemma 4.30. By Lemma 5.6, $v_{i}$ is $f$-blocked for $i<f-2$. It remains only to be shown that $v_{f-2}=v_{j}$ is $f$-blocked, but this follows from Lemma 5.5 and the fact that it neighbors $v_{2}$ and $v_{f-1}$, both of which are $f$-blocked but do not neighbor each other.

From now on in this section, we assume $\left|v_{i}\right|=2$ for $2<i<f-1$, ie case (2a) in Proposition 7.3.

Lemma 9.2 The vector $v_{f+1}$ is just right. Furthermore:

(1) If $f=4$, then $\left|v_{5}\right| \in\{3,4,6\}$.

(2) If $f=5$, then $\left|v_{6}\right| \in\{3,4,5\}$.

(3) If $f>5$, then $\left|v_{f+1}\right| \in\{3,4\}$.

Proof The only vectors of norm at least 3 in $S_{f}$ are $v_{2}$ and $v_{f-1}$, so the only possible gappy indices for $v_{f+1}$ are 1 and $f-2$. However, 1 cannot be a gappy index because then either $\left\langle v_{f+1}, v_{1}\right\rangle=-1$ or $\left\langle v_{f+1}, v_{2}\right\rangle=2$, contradicting Lemma 5.2 or Lemma 4.18, and $f-2$ cannot be a gappy index because then $\left\langle v_{f+1}, v_{f}\right\rangle=-1$, contradicting Lemma 5.2. Therefore, $v_{f+1}$ is just right.

Let $j=\min \operatorname{supp} v_{f+1}$. To avoid pairing with $v_{1}$ and $v_{f}$, either $j=0$ or $1<j<f$. When $f=4$, we get $j \in\{0,2,3\}$ and $\left|v_{5}\right| \in\{3,4,6\}$. When $f>4, j \neq 0$ because otherwise $\left\langle v_{f-1}, v_{f+1}\right\rangle=f-3>1$, contradicting Lemma 4.18. So $1<j<f$. Since $\left\langle v_{f-1}, v_{f+1}\right\rangle=f-2-j \leq 1$, we have $j \geq f-3$. If $j=f-3$, then $\left(v_{f-3} ; v_{f-4}, v_{f-2}, v_{f+1}\right)$ is a claw unless $f=5$. Therefore, $j$ is either $f-1$ or $f-2$, unless $f=5$ in which case $j$ can be $f-3$. 
Lemma 9.3 If $f=4$ and $\left|v_{5}\right|=6$, or $f=5$ and $\left|v_{6}\right|=5$, then $f+1=n+3$.

Proof In either case, $G\left(S_{f+1}\right)$ is connected, so $v_{f+2}$ is just right by Lemma 4.30. Since $\left\langle v_{f+2}, v_{f+1}\right\rangle \leq 1$ and $v_{f+2} \nsim v_{f}$, we get min supp $v_{f+2} \in\{f-1, f+1\}$. If $\min \operatorname{supp} v_{f+2}=f-1$, then we have a heavy triple $\left(v_{f-1}, v_{f+1}, v_{f+2}\right)$. If min supp $v_{f+2}=f+1$, then one obtains the claw $\left(v_{f+1} ; v_{2}, v_{f-1}, v_{f+2}\right)$.

Now, assume we are not in the above two cases, ie

$$
\left|v_{f+1}\right| \in\{3,4\} \text {. }
$$

Then $G\left(S_{f+1}\right)$ is disconnected. Let $s>f+1$ be the smallest index with $\left|v_{s}\right| \geq 3$. Note that such an index must exist, since otherwise $\widehat{G}(S)$ would be disconnected, contradicting Corollary 3.3.

Lemma 9.4 The only possible $(f+1)$-open vectors are $v_{f-2}, v_{f-1}$ and $v_{f+1}$.

Proof Since $v_{f-1}$ is unbreakable, by Lemma $5.2 v_{1}$ and $v_{f}$ are $f$-blocked. As the statement is obvious for $f=4$, we may assume $f>4$ without loss of generality. Since $\left|v_{j}\right|=2$ for $3 \leq j \leq f-2, v_{j}$ is $f$-blocked for $3 \leq j<f-2$ by Definition 5.3(3)(4) and Lemma 5.5. The following shows $v_{2}$ is $(f+1)$-blocked. Suppose $v_{j} \sim v_{2}$ for some $j>f+1$. If $2 \notin \operatorname{supp} v_{j}$, then $v_{j} \nsim v_{1}$ implies $0,1 \in \operatorname{supp} v_{j}$, which contradicts $\left|\left\langle v_{j}, v_{2}\right\rangle\right| \leq 1$. If $2 \in \operatorname{supp} v_{j}$, then $\{2,3, \ldots, f-2\} \subset \operatorname{supp} v_{j}$ because none of $2, \ldots, f-3$ can be a gappy index. We also have $f-1 \in \operatorname{supp} v_{j}$, because otherwise $\left\langle v_{j}, v_{f-1}\right\rangle \geq f-3>1$. Since $\left|v_{f}\right|=2, f-1$ is not a gappy index, so $f \in \operatorname{supp} v_{j}$. To satisfy (17) and $\left|\left\langle v_{f+1}, v_{j}\right\rangle\right| \leq 1$, it must be the case that $\left|v_{f+1}\right|=3$, but this results in a heavy triangle $\left(v_{f-1}, v_{f+1}, v_{j}\right)$.

Lemma 9.5 If $v_{s}$ is just right, then $s=f+2$. Furthermore, either $\left|v_{f+1}\right|=4$ and $\left|v_{f+2}\right|=4$, or $\left|v_{f+1}\right|=3$ and $\left|v_{f+2}\right|=5$. In either case, $f+2=n+3$.

Proof Since $\left|v_{s}\right| \geq 3$, we have $j:=\min \operatorname{supp} v_{s}<s-1$. By assumption, $\left|v_{f+2}\right|=$ $\cdots=\left|v_{s-1}\right|=2$. If $j \geq f+2$, we would have a claw $\left(v_{j} ; v_{j-1}, v_{j+1}, v_{s}\right)$. If $j=f+1$, we would have a claw $\left(v_{f+1} ; v_{f+2}, v_{s}, v_{f-1}\right.$ or $\left.v_{f-2}\right)$. Since $\left\langle v_{s}, v_{f}\right\rangle=0$, in fact $j<f$. In particular, $v_{s} \sim v_{f+1}$. By (17), $v_{f+1}$ has exactly one smaller neighbor, which is either $v_{f-1}$ or $v_{f-2}$. In either case, this is not a neighbor of $v_{s}$-in the first case, this would form a heavy triple, and in the second, it would make $\left\langle v_{s}, v_{f+1}\right\rangle \geq 2$. To avoid a claw $\left(v_{f+1} ; v_{s}, v_{f+2}, v_{f-1}\right.$ or $\left.v_{f-2}\right)$, we must have $s=f+2$. By Lemma 9.4, 
$\min \operatorname{supp} v_{f+2} \in\{f-1, f-2\}$. By (17), min supp $v_{f+1} \in\{f-1, f-2\}$. As we showed earlier in this paragraph, the smaller neighbor of $v_{f+1}$ is not adjacent to $v_{f+2}$, so $\min \operatorname{supp} v_{f+2} \neq \min \operatorname{supp} v_{f+1}$. The conclusion about $\left|v_{f+2}\right|$ follows.

To see $v_{f+2}$ is the last standard basis vector, note that $G\left(S_{f+2}\right)$ is connected. By Lemma 4.30, $v_{f+3}$ is just right. Since $\left|v_{f+2}\right| \geq 4$ and $\left\langle v_{f+2}, v_{f+3}\right\rangle \leq 1$, we see $\left|v_{f+3}\right| \in\{2,3,4\}$. Since $v_{f+3} \nsim v_{f}$, we have $v_{f+3} \neq 4$. In $G\left(S_{f+2}\right)$, there is a path containing 4 vertices $v_{f-2}, v_{f+1}, v_{f+2}, v_{f-1}$. Here $v_{f-2}, v_{f-1}$ are the two ends, and $v_{f+1}, v_{f+2}$ are in the interior. Since $\left|v_{f+3}\right| \in\{2,3\}, v_{f+3}$ is adjacent to exactly one of $v_{f+1}$ and $v_{f+2}$, and it does not neighbor $v_{f-2}$ or $v_{f-1}$. So we get a claw centered at the neighbor of $v_{f+3}$.

Lemma 9.6 If $v_{s}$ is gappy, then $f=4,\left|v_{5}\right|=4$, and

$$
v_{s}=e_{2}+e_{5}+\cdots+e_{s-1}-e_{s} .
$$

Furthermore, if $n+3>s$, then $\left|v_{s+1}\right|=3$, and $\left|v_{j}\right|=2$ for all $j>s+1$.

Proof The possible gappy indices for $v_{s}$ are $1, f$ and $f-2$. However, 1 (resp. $f$ ) cannot be a gappy index, because otherwise $\left\langle v_{s}, v_{1}\right\rangle=-1$ (resp. $\left\langle v_{s}, v_{f}\right\rangle=-1$ ) or $\left\langle v_{s}, v_{2}\right\rangle=2$ (resp. $\left\langle v_{s}, v_{f+1}\right\rangle \geq 2$ ). Therefore,

$$
v_{s}=e_{f-2}+e_{j}+\cdots+e_{s-1}-e_{s}
$$

for $f<j<s$. We have $v_{s} \sim v_{f-2}$ and $v_{s} \sim v_{f-1}$. If $\left|v_{f+1}\right|=3$, then $v_{f+1} \sim v_{f-1}$, so either $\left(v_{f-1} ; v_{1}, v_{f+1}, v_{s}\right)$ is a claw, or $\left(v_{f-1}, v_{f+1}, v_{s}\right)$ is a heavy triple. Therefore, $\left|v_{f+1}\right|=4$, and $v_{f+1} \sim v_{f-2}$. If $j>f+1$, then $\left(v_{s}, v_{f-2}, v_{f+1}, \ldots, v_{j}, v_{s}\right)$ is a cycle of length $\geq 4$. Therefore, $j=f+1$, and $v_{s} \nsim v_{f+1}$. To avoid a claw $\left(v_{f-2} ; v_{f-3}, v_{f+1}, v_{s}\right)$, we must have $f=4$ and $j=5$.

Since $v_{f-1} \sim v_{1}, v_{s}$ and $v_{2} \sim v_{f+1}, v_{s}$, we get that $v_{f-1}$ and $v_{2}$ are both $s$-blocked by Lemma 5.5 and Definition 5.3(1)-(2), hence $v_{s}$ is $s$-blocked by Lemma 5.5 and Definition 5.3(1). Lemma 5.5 and Definition 5.3(1) and (3) can further imply that $v_{j}$ is $s$-blocked for $f+1 \leq j<s-1$. Thus, $v_{s-1}$ is the only $s$-open vector, and the result follows from Lemma 5.9.

Summarizing, we have:

Proposition 9.7 Suppose that $f>3$ and $v_{f-1}$ is just right. Then $\left|v_{f-1}\right|=f-1$, and one of the following holds (other than $v_{s}$ in case (1e), all vectors are just right): 
(1) $\left|v_{j}\right|=2$ for $2<j<f-1$.

(a) $f=4, n=2,\left|v_{5}\right|=6$.

(b) $f=5, n=3,\left|v_{6}\right|=5$.

(c) $n=f-1,\left|v_{f+1}\right|=3,\left|v_{f+2}\right|=5$.

(d) $n=f-1,\left|v_{f+1}\right|=4,\left|v_{f+2}\right|=4$.

(e) $f=4,\left|v_{5}\right|=4,\left|v_{j}\right|=2$ for $5<j<s$ or $j>s+1,\left|v_{s+1}\right|=3$, and

$$
v_{s}=e_{2}+e_{5}+\cdots+e_{s-1}-e_{s} .
$$

(2) $f=5, n=2,\left|v_{3}\right|=4$.

(3) $f=6, n=3,\left|v_{3}\right|=2,\left|v_{4}\right|=3$.

The corresponding changemakers are

- $(1,1,2,3,3,10)$,

- $(1,1,2,2,5,5,14)$,

- $\left(1,1,2^{[s+1]}, 2 s+3,2 s+3,4 s+6,8 s+14\right)$,

- $\left(1,1,2^{[s+1]}, 2 s+3,2 s+3,4 s+8,8 s+14\right)$,

- $\left(1,1,2,3,3,8,8^{[s]}, 8 s+10,(8 s+18)^{[t]}\right)$,

- $(1,1,2,4,7,7)$,

- $(1,1,2,2,4,9,9)$.

\section{$10 f>3, v_{f-1}$ tight}

In this section, we assume $f>3$ and $v_{f-1}$ is tight. By Proposition 7.3, $v_{2}=e_{0}+e_{1}-e_{2}$ and $\left|v_{j}\right|=2$ for $2<j<f-1$. Since $\left\langle v_{f-1}, v_{2}\right\rangle=2=\left|v_{2}\right|-1$,

$$
\epsilon_{2}=\epsilon_{f-1} \quad \text { and } \quad A_{2} \prec A_{f-1}
$$

by Lemmas 4.14 and 4.17. Since the left endpoint of $A_{f-1}$ is $x_{*}$ (which is only contained in $A_{1}, A_{f-1}$ and $\left.A_{f}\right), A_{2}$ and $A_{f-1}$ must share the right endpoint.

Lemma 10.1 The vector $v_{f-1}$ is $f$-blocked. For $j>f$, we have $\left|\left\langle v_{j}, v_{f-1}\right\rangle\right| \in$ $\left\{0,\left|v_{j}\right|-2\right\}$. The only possible $f$-open vectors are $v_{1}, v_{f-2}$ and $v_{f}$. 
Proof Suppose for contradiction that $v_{j} \sim v_{f-1}$ for some $j>f$. Since the left endpoint of $A_{f-1}$ is $x_{*}, A_{j}$ cannot share its left endpoint with $A_{f-1}$. Since $j>f$ and $\left\langle v_{j}, v_{f-1}\right\rangle \neq 0$, we get $\left|v_{j}\right| \geq 3$. Since $z_{j} \neq z_{2}$ (Corollary 4.19) and $A_{2} \prec A_{f-1}, A_{j}$ does not share its right endpoint with $A_{f-1}$. Thus, $A_{j} \dagger A_{f-1}$. Since the left endpoint of $A_{f-1}$ is $*, A_{j}$ is distant from $A_{1}$, so $\left\langle v_{j}, v_{1}\right\rangle=0$. Since $\epsilon_{2}=\epsilon_{f-1}$, we have $\left\langle v_{j}, v_{2}\right\rangle=\left\langle v_{j}, v_{f-1}\right\rangle= \pm 1$. This implies supp $v_{j} \cap\{0,1,2\} \neq \varnothing$, so $\left\langle v_{j}, v_{f-1}\right\rangle \geq 0$. Hence, $\left\langle v_{j}, v_{2}\right\rangle=\left\langle v_{j}, v_{f-1}\right\rangle=1$. However, if $\left\langle v_{j}, v_{2}\right\rangle=1$, using $\left\langle v_{j}, v_{1}\right\rangle=0$ we get $0,1,2 \in \operatorname{supp} v_{j}$ and $\left\langle v_{j}, v_{f-1}\right\rangle \geq 3$, a contradiction. Thus, for all $j>f$, we have $v_{j} \nsim v_{f-1}$, and immediately $\left|\left\langle v_{j}, v_{f-1}\right\rangle\right| \in\left\{0,\left|v_{j}\right|-2\right\}$ by Lemma 4.14.

Since $\left|v_{i}\right|=2$ for $2<i<f-1, v_{i}$ is $f$-blocked for $3<i<f-2$ by Lemma 5.5. When $3<f-1$, by Definition 5.3(1) and (4) $v_{f-1}$ and $v_{3}$ are $f$-blocking neighbors of $v_{2}$. By Lemma 5.5, $v_{2}$ is $f$-blocked. When $3<f-2, v_{3}$ is also $f$-blocked by Lemma 5.5, since $v_{2}$ and $v_{4}$ are $f$-blocking neighbors.

Definition 10.2

$$
V_{j}:=\operatorname{supp} v_{j} \cap\{0,1, \ldots, f\} .
$$

Lemma 10.3 $V_{f+1}=\{1,2, \ldots, f-2, f\}, V_{f+2}=\{f-2\}$ or $\varnothing$, and $V_{j}=\varnothing$ for $j>f+2$.

Proof Suppose $j>f$ and $V_{j} \neq \varnothing$. Then min supp $v_{j} \in\{0,1, f-2, f-1\}$ by Lemma 10.1 and the parity condition (Lemma 3.8). We will discuss each of the four possibilities of min $\operatorname{supp} v_{j}$. Since $\left|v_{1}\right|=\left|v_{f}\right|=2$, either 0 or $f-1$ is not a gappy index. Since $\left|v_{i}\right|=2$ for $2<i<f-1$, no $i$ is a gappy index for $2 \leq i<f-2$. The following shows 1 is not a gappy index of $v_{j}$. For contradiction, suppose $1 \in$ $\operatorname{supp} v_{j}$ and $2 \notin \operatorname{supp} v_{j}$. Since $\left|\left\langle v_{j}, v_{2}\right\rangle\right| \leq 1,0 \notin \operatorname{supp} v_{j}$. Since $\left\langle v_{j}, v_{2}\right\rangle=1$, by Lemma 4.18 and (18), $\epsilon_{j}=-\epsilon_{2}=-\epsilon_{f-1}$. Since $\left\langle v_{j}, v_{1}\right\rangle=-1, A_{j}$ has $x_{0}$ as its left endpoint, so $z_{j} \in A_{f-1}$. However, since $\left\langle v_{j}, v_{f}\right\rangle \equiv\left\langle v_{j}, v_{1}\right\rangle \equiv-1(\bmod 2)$, we see $\operatorname{supp} v_{j} \cap\{f-1, f\}=\{f\}$, so $\left\langle v_{j}, v_{f-1}\right\rangle \geq 0$, contradicting $z_{j} \in A_{f-1}$ and $\epsilon_{j}=-\epsilon_{f-1}$. Thus, the only possible gappy index in $\{0,1, \ldots, f-1\}$ is $f-2$.

If min $\operatorname{supp} v_{j}=0$, then $0,1,2, \ldots, f-2 \in \operatorname{supp} v_{j}$, and $\left\langle v_{j}, v_{2}\right\rangle=1$, so $\epsilon_{j}=-\epsilon_{2}$ and $A_{j} \dagger A_{2}$ by Lemma 4.18. However, since $\left\langle v_{j}, v_{f-1}\right\rangle \geq f-1 \geq 3$, we have that $\epsilon_{j}=\epsilon_{f-1}$, contradicting (18).

If min supp $v_{j}=f-1$, then $\left\langle v_{j}, v_{f-1}\right\rangle=-1$. By Lemma 10.1, $\left|\left\langle v_{j}, v_{f-1}\right\rangle\right|=\left|v_{j}\right|-2$, so $\left|v_{j}\right|=3, A_{j} \pitchfork A_{f-1}$ and $\epsilon_{j}=-\epsilon_{f-1}$. However, $V_{j}=\{f-1, f\}$ by the parity condition, so by Lemma 4.9, $j=f+1$. Now $v_{f+1}=-e_{f+1}+e_{f}+e_{f-1}$, so 
$v_{f+1}+v_{f-1}$ is irreducible by Lemma 4.6. Since $\left\langle v_{f+1}, v_{1}\right\rangle=0, A_{f+1}$ does not contain $x_{0}$, so $\tau\left(\left[A_{f+1}\right]\right)=-\left[A_{f+1}\right]$. Since $A_{f+1} \pitchfork A_{f-1}$ and $\epsilon_{f+1}=-\epsilon_{f-1}$, up to applying $\tau, v_{f-1}+v_{f+1}$ becomes $\left[A_{f-1}\right]-\left[A_{f+1}\right]$, which is a signed sum of two distant intervals, contradicting the irreducibility of $v_{f-1}+v_{f+1}$.

If $\min \operatorname{supp} v_{j}=1$, then $1,2, \ldots, f-2 \in \operatorname{supp} v_{j}$. Since $\left\langle v_{j}, v_{f}\right\rangle \equiv\left\langle v_{j}, v_{1}\right\rangle \equiv$ $-1(\bmod 2)$ and $f-1$ is not a gappy index, $f-1 \notin \operatorname{supp} v_{j}$ and $f \in \operatorname{supp} v_{j}$. Hence, $\left\langle v_{j}, v_{f-1}\right\rangle=f-2$. By Lemma 10.1, $\left\langle v_{j}, v_{f-1}\right\rangle=\left|v_{j}\right|-2$, so $\left|v_{j}\right|=f$. By Lemma 4.9, $j-1, j \in \operatorname{supp} v_{j}$. So $\left|v_{j}\right|=f$ implies that $j=f+1$ and $V_{f+1}=\{1,2, \ldots, f-2, f\}$. If min $\operatorname{supp} v_{j}=f-2$, then $\left\langle v_{j}, v_{f}\right\rangle=\left\langle v_{j}, v_{1}\right\rangle=0$. Suppose $\left\langle v_{j}, v_{f-1}\right\rangle=0$. Then $z_{j} \notin A_{f-1}$, so $z_{j}$ is to the right of $z_{2}$ by (18). However, since $v_{2} \sim v_{3}$ and $v_{3} \nsim v_{f-1}$ when $f>4, A_{3}$ is consecutive to $A_{2}$ on the left, so $A_{3}, \ldots, A_{f-2}$ (all of norm 2) lie inside $A_{f-1}$ to the left of $z_{2}$. This contradicts $v_{f-2} \sim v_{j}$ when $f>4$. When $f=4$, the contradiction instead comes via a similar argument from the fact that $v_{j} \sim v_{2}$ but neither $z_{j} \in A_{f-1}$ nor $v_{j} \sim v_{f-1}$. The above shows $\left\langle v_{j}, v_{f-1}\right\rangle \neq 0$, hence $f-1 \notin \operatorname{supp} v_{j}$. Since $\left\langle v_{j}, v_{f}\right\rangle=0$, we have $V_{j}=\{f-2\}$.

Since $f \in \operatorname{supp} v_{f+1}$, we may conclude that $V_{f+1}=\{1,2, \ldots, f-2, f\}$.

For $j>f+1$, the above shows either $V_{j}=\varnothing$ or $V_{j}=\{f-2\}$. If $V_{j}=\{f-2\}$, then since $\left|v_{j}\right|-2=\left\langle v_{j}, v_{f-1}\right\rangle=1$, we have that $\left|v_{j}\right|=3$ and $v_{j}=-e_{j}+e_{j-1}+e_{f-2}$. Since $\left\langle v_{j}, v_{f-1}\right\rangle>0$ and $\left\langle v_{f+1}, v_{f-1}\right\rangle>0$, using Lemma 10.1 we have $\epsilon_{j}=\epsilon_{f-1}$ and $\epsilon_{f+1}=\epsilon_{f-1}$. Unless $j=f+2$ we have $\left\langle v_{j}, v_{f+1}\right\rangle=1$, thus $A_{j} \dagger A_{f+1}$ and $\epsilon_{j}=-\epsilon_{f+1}$, a contradiction.

It follows from Lemma 10.3 that

$$
v_{f+1}=e_{1}+\cdots+e_{f-2}+e_{f}-e_{f+1}
$$

and that

$$
v_{f+2}=e_{f+1}-e_{f+2} \quad \text { or } \quad e_{f-2}+e_{f+1}-e_{f+2} .
$$

Proposition 10.4 We have $\left|v_{j}\right|=2$ for $j>f+2$.

Proof By (19), $v_{f+1} \sim v_{1}, v_{f}$ and so $z_{f+1}$ is the leftmost vertex with norm $\geq 3$. In particular, $z_{f+1} \in A_{f-1}$. We also know that $z_{2}$ is the rightmost vertex in $A_{f-1}$ with norm $\geq 3$ from the beginning of this section. Thus, $A_{f+1} \subset A_{f-1}$.

By Lemma 10.3, $V_{f+2}=\{f-2\}$ or $\varnothing$. If $V_{f+2}=\varnothing$, then $v_{f+2} \sim v_{f+1}$. Since $A_{f+1} \subset A_{f-1}$ and $z_{2} \in A_{f-1}$ is to the right of $A_{f+1}, A_{f+2} \subset A_{f-1}$. If $V_{f+2}=$ $\{f-2\}$, we claim that $z_{f+2} \in A_{f-1}$. When $f>4$, since $v_{3} \nsim v_{f-1}, v_{3} \sim v_{2}$ and $A_{2}$ 
shares the right endpoint with $A_{f-1}$, we see that $A_{3}$ must abut $A_{2}$ on its left. Then since $v_{f+2} \sim v_{f-2} \sim \cdots \sim v_{3}$ and $z_{f+2}$ is the unique element with norm $\geq 3$ in the intervals $A_{f+2}, A_{f-2}, \ldots, A_{3}$, it follows that $z_{f+2}$ must be to the left of $z_{2}$. When $f=4$, this still holds because $v_{f+2} \nsim v_{f-1}$ and $v_{f+2} \sim v_{2}$. Hence $z_{f+2} \in A_{f-1}$. In either case, since $z_{2} \in A_{f-1}$ is to the right of $z_{f+2}$, we get $A_{f+2} \subset A_{f-1}$.

For any $j>f+2, V_{j}=\varnothing$ by Lemma 10.3. Since $\left\langle v_{j}, v_{f-1}\right\rangle=0$, Lemma 4.14 implies that either $A_{j}$ is distant from $A_{f-1}$ or $\left|v_{j}\right|=2$. If $A_{j}$ and $A_{f-1}$ are distant, by the result in the first paragraph in this proof $A_{f+1} \subset A_{f-1}$ and $v_{j} \nsim v_{f+1}$. When $f>4$, the argument in the second paragraph shows that $A_{f-2} \subset A_{f-1}$, and when $f=4$, we get $A_{f-2}=A_{2} \prec A_{f-1}$ by (18). So we also have $v_{j} \nsim v_{f-2}$. If $\left|v_{j}\right|=2$, then $\left\langle v_{j}, v_{f+1}\right\rangle=\left\langle v_{j}, v_{f-2}\right\rangle=0$, so $v_{j} \nsim v_{f+1}, v_{f-2}$. Thus, $v_{f+1}$ and $v_{f-2}$ are $(f+2)$-blocked. Since $\left\langle v_{1}, v_{i}\right\rangle=0$ for all $i>f+2$, we get that $v_{1}$ and $v_{f}$ are $(f+2)$-blocked. The result follows from Lemmas 10.1 and 5.7.

The corresponding changemakers are

- $\left(1,1,2^{[s+1]}, 2 s+5,2 s+5,(4 s+8)^{[t]}\right)$,

- $\left(1,1,2^{[s+1]}, 2 s+5,2 s+5,4 s+8,(4 s+10)^{[t]}\right)$ for $t>0$.

\section{$11 f=3$}

In this case, $a_{0} \geq 3, v_{2}=-e_{2}+e_{1}+2 e_{0}$ is tight by Proposition 7.2, and the left endpoint of $A_{2}$ is $x_{*}$.

Lemma 11.1 If $A_{2}$ contains $m$ distinct vertices $x_{i_{1}}, x_{i_{2}}, \ldots, x_{i_{m}}$ of norm $\geq 3$, then $S$ contains at least $m-1$ vectors $v_{j}$ such that $j>3, z_{j} \in A_{2}$ and $V_{j} \neq \varnothing$, where $V_{j}$ is as defined in Definition 10.2.

Proof Let $\Delta=\Delta(p, q), \bar{\Delta}$ be the quotient of $\Delta$ by the sublattice spanned by vectors of norm 2, and let $\pi: \Delta \rightarrow \bar{\Delta}$ be the quotient map. By the definition of $\tau=\tau_{0}$ in Definition 3.6, we have

$$
\pi \circ \tau=-\pi
$$

Since $S$ spans $\Delta$, it follows from Proposition 4.13 and (20) that $\left\{\pi\left(\left[A_{i}\right]\right)\right\}_{i=1}^{n+3}$ spans $\bar{\Delta}$. In particular, $x_{i_{1}}, x_{i_{2}}, \ldots, x_{i_{m}}$ are all linear combinations of the $\pi\left(\left[A_{i}\right]\right)$. Hence there are at least $m-1$ intervals other than $A_{2}$ containing one $x_{i_{t}}$. Since $A_{2}$ is the only 
possibly breakable interval, Corollary 4.19 implies that at least $m-1$ vertices among $x_{i_{1}}, x_{i_{2}}, \ldots, x_{i_{m}}$ are each contained in an interval $A_{j}$ for some $j>3$. For each $z_{j} \in A_{2}$, we get $\left\langle v_{j}, v_{2}\right\rangle \neq 0$ and $V_{j} \neq \varnothing$.

Lemma 11.2 We have $v_{4}=-e_{4}+e_{3}+e_{2}$ or $v_{4}=-e_{4}+e_{3}+e_{1}$. For all $j \geq 5$, $V_{j}=\varnothing$ or $\{0,1\}$ or $\{2,3\}$.

Proof Take any $j \geq 4$. Suppose $0,1 \notin V_{j}$. Then Lemma 4.11 implies that $\left\langle v_{j}, v_{3}\right\rangle=0$, so $V_{j}=\varnothing$ or $V_{j}=\{2,3\}$.

Suppose $0 \in V_{j}$. Then $1 \in V_{j}$ because $\left|v_{1}\right|=2$ implies 0 cannot be a gappy index, so $V_{j}=\{0,1,2,3\}$ or $\{0,1\}$ by Lemma 4.11. However, if $V_{j}=\{0,1,2,3\}$, then, using Lemma 4.14, $\left\langle v_{j}, v_{2}\right\rangle=2$ implies $z_{j} \in A_{2}$ and $\left|v_{j}\right|=3$ or 4 , which contradicts $V_{j}=\{0,1,2,3\}$. Hence, $V_{j}=\{0,1\}$.

Suppose $0 \notin V_{j}$ and $1 \in V_{j}$. Then Lemma 4.11 implies that $V_{j}=\{1,3\}$. Since $\left\langle v_{j}, v_{1}\right\rangle=-1, x_{0}$ is the left endpoint of $A_{j}$, and $z_{j}=x_{0} \in A_{2}$. Hence, $\left|\left\langle v_{j}, v_{2}\right\rangle\right|=$ $\left|v_{j}\right|-2$, and $\left|v_{j}\right|=3$, which only happens when $j=4$ by Lemma 4.9.

Since $3 \in \operatorname{supp} v_{4}$ by Lemma 4.9, $v_{4}=-e_{4}+e_{3}+e_{2}$ or $v_{4}=-e_{4}+e_{3}+e_{1}$.

Lemma 11.3 There is at most one $j \geq 4$ such that $V_{j}=\{2,3\}$. If $V_{j}=\{2,3\}$, then $A_{j} \dagger A_{2}$ and $\epsilon_{j}=\epsilon_{2}$. So $v_{j} \sim v_{2}$ and $z_{j} \notin A_{2}$.

Proof Suppose $V_{j}=\{2,3\}$. Since $\left\langle v_{j}, v_{2}\right\rangle=-1$, either $A_{j} \dagger A_{2}$ and $\epsilon_{j}=\epsilon_{2}$, or $A_{2} \pitchfork A_{j},\left|v_{j}\right|=3$ and $\epsilon_{j}=-\epsilon_{2}$. If $j>4$, the latter is impossible because $\left|v_{j}\right| \geq 4$ by Lemma 4.9. If $j=4$ and $A_{2} \pitchfork A_{4}$, notice that $A_{4}$ does not contain $x_{0}$ since $\left\langle v_{4}, v_{1}\right\rangle=0$, so $\tau\left(\left[A_{4}\right]\right)=-\left[A_{4}\right]$. Up to applying $\tau, v_{4}+v_{2}$ becomes $\left(\left[A_{2}\right]-\left[A_{4}\right]\right)$, which is reducible, contradicting Lemma 4.6.

Since $j=\min \left\{i|| z_{i} \mid \geq 3\right.$ and $\left.z_{i} \notin A_{2}\right\}$, there is at most one such $j$.

Lemma 11.4 If $V_{j}=\varnothing$ for $4<j \leq m$, then $\left|v_{j}\right|=2$ for $4<j \leq m$.

Proof By assumption, $v_{1}, v_{2}, v_{3}$ are $(4, m)$-blocked. Unless $m=4$ (the lemma is vacuously true when $m=4),\left|v_{5}\right|=2$ and $v_{5} \sim v_{4}$ by Lemma 4.9. If $v_{4}=-e_{4}+e_{3}+e_{2}$, then $v_{2}$ and $v_{5}$ are $(5, m)$-blocking neighbors of $v_{4}$ by Definition 5.3(1) and (4), so $v_{4}$ is $(5, m)$-blocked by Lemma 5.5. If $v_{4}=-e_{4}+e_{3}+e_{1}$, then $v_{4} \sim v_{1}$, hence $z_{4}=x_{0}$, and $v_{i} \nsim v_{4}$ unless $\left|v_{i}\right|=2$ or $z_{i} \in A_{2}$. If $\left|v_{i}\right|=2$, then $v_{i} \nsim v_{4}$ for $i>5$. For $5<i \leq m$, since $\left\langle v_{i}, v_{2}\right\rangle=0$ we get $z_{i} \notin A_{2}$. Hence, $v_{4}$ is also $(5, m)$-blocked in the case $v_{4}=-e_{4}+e_{3}+e_{1}$. The result follows from Lemma 5.7. 
Lemma 11.5 If $v_{4}=-e_{4}+e_{3}+e_{1}$, then $z_{4}=x_{0}$, and $A_{2}$ contains at least two vertices with norm $\geq 3$. Moreover, $\epsilon_{2}=\epsilon_{4}$ and $v_{2} \nsim v_{4}$.

Proof We have $\left\langle v_{4}, v_{1}\right\rangle \neq 0$, so $A_{4}$ is an interval containing $x_{0} \in A_{2}, z_{4}=x_{0}$, and $\left|v_{4}\right|=a_{0}=3$. In particular, $A_{2}$ and $A_{4}$ are not consecutive. Since $\left|v_{2}\right|=6>a_{0}, A_{2}$ contains another vertex with norm $\geq 3$. Since $\left\langle v_{4}, v_{2}\right\rangle=1=\left|v_{4}\right|-2$, we get $\epsilon_{2}=\epsilon_{4}$, $A_{2} \pitchfork A_{4}$ and $v_{2} \nsim v_{4}$.

Lemma 11.6 If $V_{j}=\{0,1\}$ for some $j>4$, then we must have $j=5$ and $v_{5}=$ $-e_{5}+e_{4}+e_{1}+e_{0}$.

Proof Suppose $V_{j}=\{0,1\}$ for some $j>4$. Then $\left\langle v_{j}, v_{2}\right\rangle=3$ and $\epsilon_{j}=\epsilon_{2}$. By Lemma 4.7, $v_{j}-v_{2}$ is irreducible, so $A_{j} \prec A_{2}$. Hence $\left|\left\langle v_{j}, v_{2}\right\rangle\right|=\left|v_{j}\right|-1$, and $\left|v_{j}\right|=4$. Depending on whether $v_{4}=-e_{4}+e_{3}+e_{1}$ or $v_{4}=-e_{4}+e_{3}+e_{2}$, we can use Lemma 11.5 or Lemma 11.3 to conclude that $\epsilon_{j}=\epsilon_{2}=\epsilon_{4}$. So $\left\langle v_{j}, v_{4}\right\rangle \leq 0$ by Lemma 4.18, and hence $4 \in \operatorname{supp} v_{j}$. Since $\left|v_{j}\right|=4$, Lemma 4.9 implies that $v_{j}$ must be $v_{5}$.

First, we consider the case where $v_{4}=-e_{4}+e_{3}+e_{2}$. Lemma 11.3 implies that $A_{2} \dagger A_{4}$. We split the case $v_{4}=-e_{4}+e_{3}+e_{2}$ into two subcases according to whether $A_{2}$ contains multiple high norm vertices.

Lemma 11.7 Suppose $v_{4}=-e_{4}+e_{3}+e_{2}$ and $a_{0}=6$. Then $\left|v_{i}\right|=2$ for all $i>4$.

Proof Since $a_{0}=\left|x_{0}\right|=\left|v_{2}\right|, x_{0}$ is the only vertex of norm $\geq 3$ in $A_{2}$. By Lemmas 11.2 and 11.3, $v_{5}=-e_{5}+e_{4}$ or $-e_{5}+e_{4}+e_{1}+e_{0}$. If $v_{5}=-e_{5}+e_{4}+e_{1}+e_{0}$, then $\left\langle v_{5}, v_{2}\right\rangle=3$, contradicting $z_{5} \notin A_{2}$. Therefore, $v_{5}=-e_{5}+e_{4}$. By Lemmas 11.2, 11.3 and 11.6, $V_{j}=\varnothing$ for all $j>4$. The result follows from Lemma 11.4.

Lemma 11.8 Suppose $v_{4}=-e_{4}+e_{3}+e_{2}$ and $a_{0}<6$. Then, $v_{5}=-e_{5}+e_{4}+e_{1}+e_{0}$, and $\left|v_{i}\right|=2$ for all $i>5$, except possibly one $v_{m}=-e_{m}+e_{m-1}+\cdots+e_{4}$.

Proof Since $\left|x_{0}\right|<\left|v_{2}\right|$, there are at least 2 vertices of norm $\geq 3$ in $A_{2}$. By Lemma 11.3, $z_{4} \notin A_{2}$. Therefore, by Lemma 11.1, there exists $V_{i} \neq \varnothing$ for some $i>4$. By Lemmas 11.2, 11.3 and 11.6, $v_{5}=-e_{5}+e_{4}+e_{1}+e_{0}$, and $V_{i}=\varnothing$ for all $i>5$. This implies $v_{2}$ is 5 -blocked by Corollary 4.20. Observe that $v_{4} \sim v_{5}$. Since $\left\langle v_{5}, v_{2}\right\rangle=3$, we have $z_{5} \in A_{2}$. By Lemma 11.3, $A_{2} \dagger A_{4}$, so $z_{4} \notin A_{2}$. If $\left|v_{i}\right|=2$ for 
some $i>6$, then $\left\langle v_{i}, v_{5}\right\rangle=0$, so $v_{5} \nsim v_{i}$. If $\left|v_{i}\right| \geq 3$, since $z_{5} \in A_{2}$ and $A_{2} \dagger A_{4}$, $v_{5} \sim v_{i}$ would imply that $z_{i} \in A_{2}$. However, for $i>6, V_{i}=\varnothing$ and $\left\langle v_{i}, v_{2}\right\rangle=0$, so $z_{i} \notin A_{2}$. Thus, $v_{5}$ is 6-blocked.

Let $m>5$ be the minimal index such that $\left|v_{m}\right| \geq 3$, if such $m$ exists. If $m=6$, $V_{m}=\varnothing$ implies $v_{6}=-e_{6}+e_{5}+e_{4}$. Assume $m>6$; then $v_{5}$ is $(m-1)-$ blocked. By Lemma 5.5, $v_{i}$ is $(m-1)$-blocked for any $5<i<m-1$. Thus, min supp $v_{m}=4$. Since $v_{m} \nsim v_{5}, 4$ is not a gappy index of $v_{m}$. Since $\left|v_{i}\right|=2$ for $5<i<m, i$ is not a gappy index for $5 \leq i<m-1$. Hence, $v_{m}$ is just right.

Since $v_{2}$ and $v_{m}$ are $m$-blocking neighbors of $v_{4}$ by Definition 5.3(1)-(2), $v_{4}$ is $m$-blocked by Lemma 5.5. We claim $v_{m-1}$ is also $m$-blocked. Suppose $v_{i} \sim v_{m-1}$ for some $i>m$, then $\left|v_{i}\right| \geq 3$. Observe that $v_{i}$ is connected to $v_{5}$ through a path of norm-2 vectors $\left(v_{i} \sim v_{m-1} \sim v_{m-2} \sim \cdots \sim v_{5}\right)$. Since $z_{5} \in A_{2}$ and $A_{2} \dagger A_{4}$, we must have $z_{i} \in A_{2}$, which contradicts $V_{i}=\varnothing$ and $\left\langle v_{i}, v_{2}\right\rangle=0$. Thus, $v_{i}$ is $m$-blocked for all $i<m$. By Lemma 5.7, $\left|v_{i}\right|=2$ for all $i>m$.

Next, we consider the case where $v_{4}=-e_{4}+e_{3}+e_{1}$.

Lemma 11.9 Suppose $v_{4}=-e_{4}+e_{3}+e_{1}$. If $v_{5}=-e_{5}+e_{4}+e_{1}+e_{0}$, then $\left|v_{i}\right|=2$ for all $i>5$.

Proof Assume $v_{5}=-e_{5}+e_{4}+e_{1}+e_{0}$. By Lemmas 11.2 and 11.6, $V_{i}=\varnothing$ or $\{2,3\}$ for $i>5$. If $V_{i}=\{2,3\}$, then by Lemma 11.3, $v_{i} \sim v_{2}, \epsilon_{i}=\epsilon_{2}$ and $z_{i} \notin A_{2}$. Since $\left\langle v_{5}, v_{2}\right\rangle=3=\left|v_{5}\right|-1, A_{2}$ and $A_{5}$ share the right endpoint and $\epsilon_{5}=\epsilon_{2}$, so $v_{i} \sim v_{5}$. Since $z_{i} \notin A_{2}$ and $z_{4}=x_{0}$ (which follows from Lemma 11.5), $v_{i} \nsim v_{4}$. Since $\left\langle v_{i}, v_{4}\right\rangle=0$, we have $4 \in \operatorname{supp} v_{i}$. Since $v_{i} \sim v_{5}$, we have $5 \notin \operatorname{supp} v_{i}$ and $\left\langle v_{i}, v_{5}\right\rangle=1$, so $\epsilon_{5}=-\epsilon_{i}$. However, this contradicts $\epsilon_{5}=\epsilon_{2}$ and $\epsilon_{i}=\epsilon_{2}$. Thus, $V_{i}=\varnothing$ for $i>5$. Therefore, $v_{1}, v_{2}$ and $v_{3}$ are 5-blocked by Corollary 4.20. For $i>5$, since $\left\langle v_{i}, v_{2}\right\rangle=0, z_{i} \notin A_{2}$. Since $z_{4}=x_{0}$, we get $v_{4} \nsim v_{i}$ for $i>5$. Since $v_{1}, v_{2}, v_{3}$ and $v_{4}$ are 5 -blocked, $\left|v_{i}\right|=2$ for all $i>5$ by Lemma 5.7.

Lemma 11.10 Suppose $v_{4}=-e_{4}+e_{3}+e_{1}$. If there exists $V_{m}=\{2,3\}$, then $v_{m}=-e_{m}+e_{m-1}+\cdots+e_{2}$ and $\left|v_{i}\right|=2$ for all $i>4, i \neq m$.

Proof By Lemmas 11.2, 11.3, 11.6 and 11.9, $V_{i}=\varnothing$ for all $i>4, i \neq m$. By Lemma 11.4, $\left|v_{i}\right|=2$ for $4<i<m$. Hence, $i$ is not a gappy index for $4 \leq i<m-1$. Recall that $z_{4}=x_{0}$ from Lemma 11.5. By Lemma 11.3, $z_{m} \notin A_{2}$, so $v_{m} \nsim v_{4}$, and $4 \in \operatorname{supp} v_{m}$. Therefore, $v_{m}=-e_{m}+e_{m-1}+\cdots+e_{2}$. 
Since $V_{i}=\varnothing$ for $i>m$, we get that $v_{1}, v_{2}, v_{3}$ are $m$-blocked. Take any $4 \leq j<m$. Observe that $v_{j}$ is connected to $v_{4}$ through a path of norm-2 vectors, and recall that $z_{4}=x_{0}$, so each $A_{j}$ is contained in the interior of $A_{2}$. For any $i>m, v_{i} \nsim v_{j}$ unless $\left|v_{i}\right|=2$ or $z_{i} \in A_{2}$. If $\left|v_{i}\right|=2$, we have $\left\langle v_{i}, v_{j}\right\rangle=0$, so $v_{i} \nsim v_{j}$. If $z_{i} \in A_{2}$, then $\left\langle v_{i}, v_{2}\right\rangle \neq 0$, so $V_{i} \neq \varnothing$, a contradiction. So $v_{j}$ is $m$-blocked for $4 \leq j<m$. By Lemma 5.7, $\left|v_{i}\right|=2$ for all $i>m$.

Proposition 11.11 When $f=3$ and $v_{2}$ is tight, one of the following holds:

(1) $v_{4}=-e_{4}+e_{3}+e_{2}$ and $\left|v_{i}\right|=2$ for all $i>4$.

(2) $v_{4}=-e_{4}+e_{3}+e_{2}, v_{5}=-e_{5}+e_{4}+e_{1}+e_{0}$ and $\left|v_{i}\right|=2$ for all $i>5$ except possibly one $v_{m}=-e_{m}+e_{m-1}+\cdots+e_{4}$.

(3) $v_{4}=-e_{4}+e_{3}+e_{1}, v_{5}=-e_{5}+e_{4}+e_{1}+e_{0}$ and $\left|v_{i}\right|=2$ for all $i>5$.

(4) $v_{4}=-e_{4}+e_{3}+e_{1}$ and $\left|v_{i}\right|=2$ for all $i>4$ except possibly one $v_{m}=$ $-e_{m}+e_{m-1}+\cdots+e_{2}$.

Proof When $v_{4}=-e_{4}+e_{3}+e_{2}$ and $a_{0}=6$, item (1) holds by Lemma 11.7. When $v_{4}=-e_{4}+e_{3}+e_{2}$ and $a_{0}<6$, item (2) holds by Lemma 11.8. When $v_{4}=-e_{4}+e_{3}+e_{1}$ and $V_{5}=\{0,1\}$, item (3) holds by Lemma 11.9. When $v_{4}=-e_{4}+e_{3}+e_{1}$ and $V_{5}=\varnothing$ or $\{2,3\}$, item (4) holds by Lemma 11.10 if there exists $V_{m}=\{2,3\}$, or by Lemma 11.4 if there is no $V_{m}=\{2,3\}$.

The corresponding changemakers are

- $\left(1,1,3,3,4^{[s]},(4 s+6)^{[t]}\right)$ for $s+t>0$,

- $\left(1,1,3,3,6,8^{[s]},(8 s+6)^{[t]}\right)$,

- $\left(1,1,3,3,4,6^{[s]}\right)$ for $s>0$.

\section{Determining $p$ and $q$}

Having classified all the $(n+3)$-dimensional D-type lattices which are changemaker lattices, we now aim to concretely compute the pairs $(p, q)$ for these lattices. More precisely, we will give a list $\mathcal{P}^{-}$of prism manifolds with the property that if positive surgery on some knot in $S^{3}$ results in a prism manifold $P(p, q)$ with $q<0$, then $P(p, q) \in \mathcal{P}^{-}$.

Recall from Section 2 that a prism manifold $P(p, q)$ is the boundary of a sharp 4manifold $X(p, q)$ when $q<0$. The homology group $H_{2}(X(p, q))$ equipped with the 
inner product $-Q_{X(p, q)}$ becomes a lattice isomorphic to $\Delta(p, q)$. From the integers $a_{0}, a_{1}, \ldots, a_{n}$ in (10), we can recover $p$ and $q$ using (7).

Our strategy to determine $p$ and $q$ is as follows. Let $S=\left\{v_{1}, \ldots, v_{n+3}\right\}$ be the standard basis for a changemaker lattice that is isomorphic to a D-type lattice. Convert $S$ into a vertex basis, denoted $S^{*}=\left\{v_{1}^{*}, \ldots, v_{n+3}^{*}\right\}$; that is, we need to do a change of basis to convert $S$ to a vertex basis; see the example below for instance. (Also, all the linear transformations needed to change the standard bases corresponding to the changemaker vectors to vertex bases are presented in Table 4, part I.) Using the results of Section 4, the $a_{i}$ will be recovered. We can then get the pair $(p, q)$ using (7). The following lemma helps to simplify some involved computations of continued fractions that we will face. These properties are the first two items of [10, Lemma 9.5].

Lemma 12.1 For integers $r, s, t \geq 2$,

(1) $\left[\ldots, r, 2^{[s]}, t, \ldots\right]^{-}=[\ldots, r-1,-(s+1), t-1, \ldots]^{-}$, and

(2) $\left[\ldots, s, 2^{[t]}\right]^{-}=[\ldots, s-1,-(t+1)]^{-}$.

Example 12.2 We illustrate how to obtain the pair $(p, q)$ in the case when $f=3$, $v_{2}$ is tight, and $\left|v_{4}\right|=3$ (cf Proposition 11.11(2)). We point out that the chosen changemaker corresponds to the parameters $t=0$ and $s \geq 1$. Let $S=\left\{v_{1}, \ldots, v_{n+3}\right\}$ denote the standard basis for the changemaker lattice $L=(\sigma)^{\perp}$. One easily computes

$$
\sigma=\left(1,1,3,3,6,8^{[n-1]}\right), \quad n \geq 2 .
$$

From the pairing graph it follows that $S$ is not a vertex basis. Taking

$$
v_{2}^{*}=v_{2}-v_{1}-v_{5}-\cdots-v_{n+3},
$$

we get that

$$
S^{*}=\left\{v_{1}, v_{3}\right\} \cup\left\{v_{2}^{*}, v_{n+3}, \ldots, v_{5}, v_{4}\right\}
$$

is indeed a vertex basis with norms given by the tuple

$$
V^{*}=\left(2,2,4,2^{[n-2]}, 4,3\right), \quad n \geq 2 .
$$

Using Lemma 12.1 together with (7), we get

$$
\frac{-q}{p}=\left[4-1,2^{[n-2]}, 4,3\right]^{-}=\frac{16(n-1)+14}{8(n-1)+3} .
$$

Observe that $-q=2(p+4)$. Moreover, looking at the denominator of the right-hand side of $(21)$, we get that $p \equiv 3(\bmod 8)$. Since $n \geq 2$, we have $p \geq 11$. The case in the example is in Table 1 , type 4 with $r=2(n-1)+1, p=4 r-1$ and $-q=8 r+6$. 


\begin{tabular}{cll}
\hline type & $P(p, q)$ & $\begin{array}{l}\text { range of parameters } \\
(p \text { and } r \text { are always odd, } p>1)\end{array}$ \\
\hline 1A & $P\left(p,-\frac{1}{2}\left(p^{2}-3 p+4\right)\right)$ & $p \geq 7$ \\
1B & $P\left(p,-\frac{1}{22}\left(p^{2}-3 p+4\right)\right)$ & $p \equiv 17$ or $19(\bmod 22), \quad p>22$ \\
2 & $P\left(p,-\frac{1}{|4 r+2|}\left(r^{2} p+1\right)\right)$ & $r \equiv-1(\bmod 4), \quad r \neq-1,3$, \\
& & $p \equiv 2 r-3(\bmod 4 r+2)$ \\
3A & $P\left(p,-\frac{1}{2 r}(p+1)(p+4)\right)$ & $r \geq 1$, \\
& $P\left(p,-\frac{1}{2 r}(p+1)(p+4)\right)$ & $r \geq 5$, \\
3B & $P \equiv r-4(\bmod 2 r), \quad p \geq 3 r-4$ \\
& $P\left(p,-\frac{1}{2 r^{2}}\left((2 r+1)^{2} p+1\right)\right)$ & $r \neq-3,-1,1$, \\
4 & $p \equiv 4 r-1\left(\bmod 2 r^{2}\right), \quad p \geq 4 r-1$ \\
& $P\left(p,-\frac{1}{r^{2}-2 r-1}\left(r^{2} p+1\right)\right)$ & $r \neq 1$, \\
5 & $P(11,-30), P(17,-31)$, & $p \equiv 2 r-5\left(\bmod r^{2}-2 r-1\right), \quad p \geq 2 r-5$ \\
sporadic & $P(13,-47), P(23,-64)$ &
\end{tabular}

Table 1: $\mathcal{P}^{-}$, table of $P(p, q)$ that are realizable, $q<0$.

Similar computations for the D-type changemaker lattices give prism manifolds $P(p, q)$, so that up to reparametrization, each falls into one of the families in Table 1. We shall denote the collection of these families by $\mathcal{P}^{-}$. Here we divide the families so that each changemaker vector corresponds to a unique family. The detailed correspondence between the changemaker vectors and $P(p, q)$ can be found in Table 4. It should be noted that, as discussed in Section 2, the positive integer $p$ is always odd.

\section{Primitive/Seifert-fibered knots admitting prism manifold surgeries}

In the previous sections, we provided a list of changemaker vectors in $\mathbb{Z}^{n+4}$ whose orthogonal complements are isomorphic to D-type lattices. This list gave rise to a collection $\mathcal{P}^{-}$of prism manifolds. Let $\sigma$ be a changemaker vector that corresponds to $P(p, q) \in \mathcal{P}^{-}$. We want find a knot $K_{\sigma} \subset S^{3}$ on which some surgery yields $P(p, q)$. All known such knots are P/SF knots, which are classified in the forthcoming work of Berge and Kang [2]; we outline their work here based on the preprint Berge shared with us. To start, we need to recall some definitions. 
Definition 13.1 A Seifert-fibered 3-manifold is a 3-manifold together with a decomposition into disjoint simple closed curves, called fibers, such that each fiber has a tubular neighborhood that forms a standard fibered torus. A standard fibered torus corresponding to a pair of coprime integers $(m, n)$, with $m$ positive, is the surface bundle of the diffeomorphism of a disk given by rotation by an angle of $2 \pi n / m$, with the natural fibering by circles. If $m>1$, then the middle fiber is called singular.

Definition 13.2 Let $H$ be a genus-two handlebody. A simple closed curve $c \subset \partial H$ is primitive in $H$ if $H[c]$, the manifold obtained by adding a two-handle to $H$ along $c$, is a solid torus. A simple closed curve $c \subset \partial H$ is Seifert-fibered in $H$ if $H[c]$ is a Seifert-fibered space.

Definition 13.3 Let $\Sigma$ denote the genus-two Heegaard surface of the standard genustwo Heegaard splitting of $S^{3}=H \cup_{\Sigma} H^{\prime}$. A knot $K \subset S^{3}$ is called primitive/Seifertfibered, denoted P/SF, if it has a presentation as a simple closed curve on $\Sigma$ such that $K$ is primitive in $H^{\prime}$ and Seifert-fibered in $H$. The isotopy class in $\partial \nu(K)$ of the curves in $\partial v(K) \cap \Sigma$ is called the surface slope of $K$ with respect to $\Sigma$. Below, we will use $\gamma \in \mathbb{Z}$ to denote the surface slope.

P/SF knots are generalizations of doubly primitive knots (ie knots that can be isotoped to lie on $\Sigma$ and are primitive in both handlebodies $H$ and $H^{\prime}$ ) studied by Berge [1]. In [6], Dean studied the Dehn surgery on P/SF knots along their surface slopes. He proved that the surface slope surgery on a P/SF knot results in either a Seifert-fibered manifold or a connected sum of lens spaces [6, Proposition 2.3]. Also, Eudave Muñoz proved that the latter case does not happen for hyperbolic P/SF knots [9, Theorem 4].

Berge and Kang, in [2], classified all P/SF knots. Furthermore, given a P/SF knot $K$, they specified the indices of the singular fibers of the Seifert-fibered manifold obtained from the surface slope surgery on $K$.

Recall that for a pair of relatively prime integers $p>1$ and $q$, a prism manifold $P(p, q)$ is a Seifert-fibered manifold with base orbifold $S^{2}$ and three singular fibers of index

$$
2,2, p \text {. }
$$

Using (2), if $P(p, q)$ arises from the surface slope surgery on a P/SF knot, then

$$
\gamma=\text { surface slope }= \pm 4 q .
$$




\begin{tabular}{cll}
\hline type & $P(p, q)$ & $\begin{array}{l}\text { range of parameters } \\
(p \text { and } r \text { are always odd, } p>1)\end{array}$ \\
\hline 1A & $P\left(p, \frac{1}{2}\left(p^{2}+3 p+4\right)\right)$ & \\
1B & $P\left(p, \frac{1}{22}\left(p^{2}+3 p+4\right)\right)$ & $p \equiv 5$ or $3(\bmod 22)$ \\
2 & $P\left(p, \frac{1}{|4 r+2|}\left(r^{2} p-1\right)\right)$ & $r \equiv-1(\bmod 4), \quad p \equiv-2 r+3(\bmod 4 r+2)$ \\
3A & $P\left(p, \frac{1}{2 r}(p-1)(p-4)\right)$ & $p \equiv 1(\bmod 2 r)$ \\
3B & $P\left(p, \frac{1}{2 r}(p-1)(p-4)\right)$ & $p \equiv r+4(\bmod 2 r)$ \\
4 & $P\left(p, \frac{1}{2 r^{2}}\left((2 r+1)^{2} p-1\right)\right)$ & $p \equiv-4 r+1\left(\bmod 2 r^{2}\right)$ \\
5 & $P\left(p, \frac{1}{r^{2}-2 r-1}\left(r^{2} p-1\right)\right)$ & $r \neq 1, \quad p \equiv-2 r+5\left(\bmod r^{2}-2 r-1\right)$ \\
sporadic & $P(11,19), P(13,34)$ &
\end{tabular}

Table 2: $\mathcal{P}^{+}$, table of $P(p, q)$ that are realizable, $q>0$.

The surface slope given in [2] is not necessarily positive, while we only consider positive surgery. To get a knot with positive prism manifold surgery, we need to take the mirror image of the Berge-Kang knot if the surface slope is negative. Suppose the surface slope surgery on $K$ results in $P(p, q)$. Then $p$ is the index of the singular fiber with odd index, and $q$ satisfies (23). With a bit more work, we can determine the sign of $q$. Since Berge and Kang's paper [2] is not publicly available, we will omit the

\begin{tabular}{lcll}
\hline prism manifold & type & changemaker & braid word \\
\hline$P(3,-14)$ & $3 \mathrm{~A}$ & $(1,1,2,5,5)$ & $\left(\sigma_{1} \cdots \sigma_{11}\right)^{5}\left(\sigma_{1}\right)^{-2}$ \\
& 5 & $(1,1,3,3,6)$ & $\begin{array}{l}\left(\sigma_{3} \sigma_{4} \sigma_{5} \sigma_{2} \sigma_{3} \sigma_{4} \sigma_{1} \sigma_{2} \sigma_{3}\right)^{3}\left(\sigma_{1} \sigma_{2}\right)^{10} \\
((19,3)-\text { cable of } T(3,2))\end{array}$ \\
\hline$P(11,-18)$ & 2 & $(1,1,2,4,5,5)$ & $\left(\sigma_{1} \cdots \sigma_{13}\right)^{5} \sigma_{1} \sigma_{2}$ \\
& 3B & $(1,1,3,3,4,6)$ & $\left(\sigma_{1} \cdots \sigma_{5}\right)^{10}\left(\sigma_{1} \sigma_{2} \sigma_{3}\right)^{3}$ \\
\hline $\begin{array}{l}P(8 s+3,-(16 s+14)) \\
s \geq 1\end{array}$ & $4 \mathrm{~A}$ & $\left(1,1,2,5,5,8^{[s]}\right)$ & $\left(\sigma_{1} \cdots \sigma_{7}\right)^{5+8 s}\left(\sigma_{7} \cdots \sigma_{1}\right)^{2}$ \\
& spor & $\left(1,1,3,3,6,8^{[s]}\right)$ & $\left(\sigma_{1} \cdots \sigma_{13}\right)^{8}\left(\sigma_{1} \cdots \sigma_{7}\right)^{8 s-7}$ \\
& $s=1$ & $(1,1,2,4,7,7)$ & $\left(\sigma_{1} \cdots \sigma_{17}\right)^{7}\left(\sigma_{2} \sigma_{1}\right)^{-2}$ \\
\hline
\end{tabular}

Table 3: Prism manifolds arising from multiple changemaker vectors. 


\begin{tabular}{|c|c|c|}
\hline $\operatorname{Prop}^{n}$ & changemaker vector & $\begin{array}{l}\text { vertex basis (with } x_{*}, x_{* *} \text { omitted) } \\
\left\{x_{0}, \ldots, x_{n}\right\}\end{array}$ \\
\hline \multirow[t]{7}{*}{9.7} & $(1,1,2,4,7,7)$ & $\left\{v_{4},-v_{3}, v_{2}\right\}$ \\
\hline & $(1,1,2,3,3,10)$ & $\left\{v_{3},-v_{5}, v_{2}\right\}$ \\
\hline & $(1,1,2,2,4,9,9)$ & $\left\{v_{5},-v_{4},-v_{2},-v_{3}\right\}$ \\
\hline & $(1,1,2,2,5,5,14)$ & $\left\{v_{4},-v_{6},-v_{2},-v_{3}\right\}$ \\
\hline & $\left(1,1,2^{[s+1]},(2 s+3)^{[2]}, 4 s+6,8 s+14\right)$ & $\left\{v_{s+3}, v_{s+5},-v_{s+6},-v_{s+2} \ldots,-v_{2}\right\}$ \\
\hline & $\left(1,1,2^{[s+1]},(2 s+3)^{[2]}, 4 s+8,8 s+14\right)$ & $\left\{v_{s+3}, v_{s+6},-v_{s+5}\right.$ \\
\hline & $\left(1,1,2,3,3,8,8^{[s]}, 8 s+10,(8 s+18)^{[t]}\right)$ & $\begin{array}{r}\left\{v_{3},-v_{s+6},-v_{2},-v_{5}, \ldots,-v_{s+5}\right. \\
\left.-v_{s+7}, \ldots,-v_{s+t+6}\right\}\end{array}$ \\
\hline \multirow[t]{2}{*}{6.5} & $\left(1,1,1,1,2^{[s]}\right), \quad s>0$ & $\left\{v_{2}, v_{4}, v_{5}, \ldots, v_{s+3}\right\}$ \\
\hline & $\left(1,1,1,1,4^{[s]}, 4 s+2,(4 s+6)^{[t]}\right)$ & $\begin{aligned}\left\{v_{2}, v_{s+4},-v_{4}, \ldots,-v_{s+3}\right. & \\
& \left.-v_{s+5}, \ldots,-v_{t+s+4}\right\}\end{aligned}$ \\
\hline \multirow[t]{3}{*}{10.4} & $\begin{array}{l}\left(1,1,2^{[s+1]},(2 s+5)^{[2]},(4 s+8)^{[t]}\right) \\
t>0\end{array}$ & $\begin{array}{l}\left\{v_{s+5}, \ldots, v_{t+s+4}\right. \\
\quad v_{s+3}-v_{[1, s+2]}-v_{[s+5, t+s+4]}\end{array}$ \\
\hline & $\left(1,1,2^{[s+1]},(2 s+5)^{[2]}\right)$ & $\left\{v_{s+3}-v_{[1, s+2]}, v_{s+2}, \ldots, v_{2}\right\}$ \\
\hline & $\begin{array}{l}\left(1,1,2^{[s+1]},(2 s+5)^{[2]}, 4 s+8,(4 s+10)^{[t]}\right) \\
t>0\end{array}$ & $\begin{array}{r}\left\{v_{s+5}, v_{s+3}-v_{[1, s+2]}-v_{[s+5, s+t+5]}\right. \\
\left.v_{s+t+5}, \ldots, v_{s+6}, v_{s+2}, \ldots, v_{2}\right\}\end{array}$ \\
\hline \multirow[t]{4}{*}{11.11} & $\left(1,1,3,3,4^{[s]},(4 s+6)^{[t]}\right), \quad s>0$ & $\begin{array}{r}\left\{v_{4}, \ldots, v_{s+3}, v_{2}-v_{1}-v_{[4, s+3]}\right. \\
\left.v_{s+4}, \ldots, v_{t+s+3}\right\}\end{array}$ \\
\hline & $\left(1,1,3,3,6^{[t]}\right), \quad t>0$ & $\left\{v_{2}-v_{1}, v_{4}, \ldots, v_{t+3}\right\}$ \\
\hline & $\left(1,1,3,3,4,6^{[s]}\right), \quad s>0$ & $\left\{v_{4}, v_{2}-v_{1}-v_{[4, s+4]}, v_{s+4}, \ldots, v_{5}\right\}$ \\
\hline & $\left(1,1,3,3,6,8^{[s]},(8 s+6)^{[t]}\right), \quad s>0$ & $\left\{\begin{aligned}\left\{v_{2}-v_{1}-v_{[5, s+4]},\right. & v_{s+4}, \ldots, v_{4} \\
& \left.v_{s+5}, \ldots, v_{t+s+4}\right\}\end{aligned}\right.$ \\
\hline \multirow[t]{4}{*}{8.1} & $\left(1,1,2,2,2^{[s]},(2 s+3)^{[2]}\right), \quad s>0$ & $\left\{v_{s+4}, v_{3}, v_{4}, \ldots, v_{s+3},-v_{[2, s+3]}\right\}$ \\
\hline & $\left(1,1,2,4,4^{[s]},(4 s+5)^{[2]},(8 s+14)^{[t]}\right)$ & $\begin{aligned} &\left\{v_{s+4},-v_{2}, v_{3}, \ldots, v_{s+3}\right. \\
&\left.v_{s+6}, \ldots, v_{t+s+5}\right\}\end{aligned}$ \\
\hline & $\begin{array}{l}\left(1,1,2,2,4^{[s]},(4 s+3)^{[2]},(8 s+10)^{[t]}\right), \\
s>0\end{array}$ & $\begin{array}{l}\left\{v_{s+4}+v_{3},-v_{3},-v_{2},-v_{4}\right. \\
\left.\quad \ldots,-v_{s+3},-v_{s+6}, \ldots,-v_{t+s+5}\right\}\end{array}$ \\
\hline & $\left(1,1,2,2,3^{[2]}, 10^{[t]}\right)$ & $\left\{v_{4}+v_{3},-v_{3},-v_{2},-v_{6}, \ldots,-v_{t+5}\right\}$ \\
\hline
\end{tabular}

Table 4: D-type changemakers vs prism manifolds, I.

process of determining the sign here. Instead, we directly give the list $\mathcal{P}^{+}$of realizable $P(p, q)$ with $q>0$ in Table 2 without proof, and will justify this list in future work. Unlike Table 1, we do not have the notion of changemaker vector here. We divide these 


\begin{tabular}{|c|c|c|c|}
\hline Prop $^{n}$ & vertex norms $\left\{a_{0}, \ldots, a_{n}\right\}$ & prism manifold parameters & $\mathcal{P}^{-}$type \\
\hline \multirow[t]{7}{*}{9.7} & $\{4,4,3\}$ & $p=11, \quad-q=30$ & spor \\
\hline & $\{3,6,3\}$ & $p=17, \quad-q=31$ & spor \\
\hline & $\{5,3,3,2\}$ & $p=13, \quad-q=47$ & spor \\
\hline & $\{4,5,3,2\}$ & $p=23, \quad-q=64$ & spor \\
\hline & $\left\{s+3,3,5,2^{[s]}, 3\right\}$ & $p=22 s+39, \quad-q=22 s^{2}+75 s+64$ & $1 \mathrm{~B}$ \\
\hline & $\left\{s+3,4,4,2^{[s]}, 3\right\}$ & $p=22 s+41, \quad-q=22 s^{2}+79 s+71$ & $1 \mathrm{~B}$ \\
\hline & $\left\{3, s+3,3,4,2^{[s]}, 3,2^{[t-1]}\right\}$ & $\begin{aligned} p & =2 r^{2} t+2 r^{2}+4 r-1, \\
-q & =(2 r+1)^{2}(t+1)+8 r+6, \\
r & =-(2 s+5)\end{aligned}$ & 4 \\
\hline \multirow[t]{2}{*}{6.5} & $\left\{2,3,2^{[s-1]}\right\}$ & $p=2 s+1, \quad-q=s+1, \quad r=-1$ & 5 \\
\hline & $\left\{2, s+3,5,2^{[s-1]}, 3,2^{[t-1]}\right\}$ & $\begin{aligned} p & =r^{2}-6+\left(r^{2}-2 r-1\right) t \\
-q & =r^{2}(t+1)+2 r-1 \\
r & =-(2 s+3)\end{aligned}$ & 5 \\
\hline \multirow[t]{3}{*}{10.4} & $\left\{s+4,2^{[t-1]}, 4,2^{[s]}, 3\right\}$ & $\begin{aligned} p & =(2 t+1)(2 s+4)-1 \\
-q & =(s+2)((2 t+1)(2 s+4)+3) \\
r & =2 t+1\end{aligned}$ & $3 \mathrm{~A}$ \\
\hline & $\left\{s+6,2^{[s]}, 3\right\}$ & $\begin{array}{l}p=2 s+3,-q=2 s^{2}+11 s+14 \\
r=1\end{array}$ & $3 \mathrm{~A}$ \\
\hline & $\left\{s+4,3,2^{[t-1]}, 3,2^{[s]}, 3\right\}$ & $\begin{aligned} p & =(2 t+3)(2 s+5)-4 \\
-q & =(2 s+5)((2 t+3) s+5 t+6) \\
r & =2 t+3\end{aligned}$ & $3 B$ \\
\hline
\end{tabular}

Table 4: D-type changemakers vs prism manifolds, II.

prism manifolds into families in such a way that it reflects the "symmetry" between the two tables, noting that a knot may correspond to more than one family in Table 2. We take $\mathcal{P}=\mathcal{P}^{-} \cup \mathcal{P}^{+}$.

\subsection{Manifolds corresponding to distinct changemaker vectors}

As we mentioned before, the families in Table 1 are divided so that every changemaker vector corresponds to a unique family. However, a lattice $\Delta(p, q)$ may be isomorphic to the orthogonal complements of different changemaker vectors. Thus the corresponding prism manifold $P(p, q)$ lies in different families, and not just one. In Table 3, we list all such $P(p, q)$. Each of these prism manifolds is contained in two families, except that $P(11,-30)$ is contained in three families. If a P/SF knot admits a surgery to such 


\begin{tabular}{|c|c|c|c|}
\hline Prop $^{n}$ & vertex norms $\left\{a_{0}, \ldots, a_{n}\right\}$ & prism manifold parameters & $\mathcal{P}^{-}$type \\
\hline \multirow[t]{4}{*}{11.11} & $\left\{3,2^{[s-1]}, 5, s+3,2^{[t-1]}\right\}$ & $\begin{aligned} p & =\left(r^{2}-2 r-1\right) t+2 r-5 \\
-q & =r^{2} t+2 r-1, \quad r=2 s+3\end{aligned}$ & 5 \\
\hline & $\left\{6,3,2^{[t-1]}\right\}$ & $p=2 t+1, \quad-q=9 t+5, \quad r=3$ & 5 \\
\hline & $\left\{3,3,2^{[s-1]}, 4\right\}$ & $p=6 s+5, \quad-q=9 s+9, \quad r=2 s+3$ & $3 B$ \\
\hline & $\left\{4,2^{[s-1]}, 4,3, s+2,2^{[t-1]}\right\}$ & $\begin{aligned} p & =2 r^{2} t+4 r-1 \\
-q & =(2 r+1)^{2} t+8 r+6, \quad r=2 s+1\end{aligned}$ & 4 \\
\hline \multirow[t]{4}{*}{8.1} & $\left\{s+3,2^{[s+1]}, 3\right\}$ & $p=2 s+5, \quad-q=2 s^{2}+7 s+7$ & $1 \mathrm{~A}$ \\
\hline & $\left\{s+3,3,4,2^{[s]}, 4,2^{[t-1]}\right\}$ & $\begin{aligned} p & =16 t s+30 t+8 s+11 \\
-q & =16 t s^{2}+56 t s+8 s^{2}+24 s+49 t+18 \\
r & =4 s+7\end{aligned}$ & 2 \\
\hline & $\left\{s+3,2,3,3,2^{[s-1]}, 4,2^{[t-1]}\right\}$ & $\begin{aligned} p & =16 t s+18 t+8 s+5 \\
-q & =16 t s^{2}+40 t s+8 s^{2}+25 t+16 s+7 \\
r & =-4 s-5\end{aligned}$ & 2 \\
\hline & $\left\{3,2,3,5,2^{[t-1]}\right\}$ & $p=18 t+5, \quad-q=25 t+7, \quad r=-5$ & 2 \\
\hline
\end{tabular}

Table 4: D-type changemakers vs prism manifolds, III.

a $P(p, q)$, we require extra information in order to detect the changemaker vector that corresponds to this knot. The information we will collect is the Alexander polynomial. Let $\sigma$ be a changemaker vector such that $\Delta(p, q) \cong(\sigma)^{\perp}$. Assume that $\sigma$ corresponds to a knot $K$ admitting a surgery to $P(p, q)$. Using Lemma 2.10, we can compute the Alexander polynomial $\Delta_{K}(T)$. We will explicitly exhibit a P/SF knot $K_{0}$ admitting a surgery to $P(p, q)$, and directly compute $\Delta_{K_{0}}(T)$ to check that it is equal to the predicted Alexander polynomial $\Delta_{K}(T)$. So $K_{0}$ matches with $\sigma$.

\subsection{Proofs of the main results}

This subsection is devoted to the proof of Theorems 1.2 and 1.6.

Proof of Theorem 1.6 If $\Delta(p, q)$ is isomorphic to a changemaker lattice $L$ then it belongs to the families classified in Sections 6-11. As in Section 12, we can find a pair $\left(p^{\prime}, q^{\prime}\right)$ such that $L$ is isomorphic to $\Delta\left(p^{\prime}, q^{\prime}\right)$, and $P\left(p^{\prime}, q^{\prime}\right)$ is in $\mathcal{P}^{-}$. Using Proposition 3.12, the result follows.

Table 4 parts I, II and III collect all results, matching each of the changemakers deduced from Propositions $6.5,8.1,9.7,10.4$ and 11.11 to one pair $(p, q) \in \mathcal{P}^{-}$. In all families of changemaker vectors, the parameters $s$ and $t$ are assumed to be nonnegative, unless 
otherwise indicated. In Table 4, $v_{[a, b]}$ means $v_{a}+v_{a+1}+\cdots+v_{b}$ for $a<b$. All vertex bases are presented in the form $\left\{x_{0}, x_{1}, \ldots, x_{n}\right\}$. A superscript [-1] at an element in the sequence of vertex norms means that the sequence is truncated just before the element preceding it. For example, the sequence $\left\{3, s+3,3,4,2^{[s]}, 3,2^{[t-1]}\right\}$ becomes $\left\{3, s+3,3,4,2^{[s]}\right\}$ when $t=0$.

Proof of Theorem 1.2 It follows from the discussion in this and the last subsections together with Theorem 1.6 that if $P(p, q) \cong S_{4|q|}^{3}(K)$, then $P(p, q)$ is in $\mathcal{P}^{-}$, and $\Delta_{K}(t)$ is determined by $P(p, q)$ and the family containing it. By [19], the knot Floer homology group of $K$ is also determined.

\section{References}

[1] J Berge, Some knots with surgeries yielding lens spaces, preprint (1990) arXiv

[2] J Berge, S Kang, The hyperbolic $\mathrm{P} / \mathrm{P}, \mathrm{P} / \mathrm{SF}_{d}$, and $\mathrm{P} / \mathrm{SF}_{m}$ knots in $S^{3}$, preprint (2014)

[3] S A Bleiler, C D Hodgson, Spherical space forms and Dehn filling, Topology 35 (1996) 809-833 MR

[4] S Boyer, X Zhang, Finite Dehn surgery on knots, J. Amer. Math. Soc. 9 (1996) 10051050 MR

[5] M Culler, C M Gordon, J Luecke, P B Shalen, Dehn surgery on knots, Ann. of Math. 125 (1987) 237-300 MR

[6] J C Dean, Small Seifert-fibered Dehn surgery on hyperbolic knots, Algebr. Geom. Topol. 3 (2003) 435-472 MR

[7] M I Doig, On the number of finite p/q-surgeries, Proc. Amer. Math. Soc. 144 (2016) 2205-2215 MR

[8] S K Donaldson, An application of gauge theory to four-dimensional topology, J. Differential Geom. 18 (1983) 279-315 MR

[9] M Eudave Muñoz, Band sums of links which yield composite links: the cabling conjecture for strongly invertible knots, Trans. Amer. Math. Soc. 330 (1992) 463-501 MR

[10] J E Greene, The lens space realization problem, Ann. of Math. 177 (2013) 449-511 MR

[11] J E Greene, L-space surgeries, genus bounds, and the cabling conjecture, J. Differential Geom. 100 (2015) 491-506 MR

[12] L Gu, Integral finite surgeries on knots in $S^{3}$, preprint (2014) arXiv

[13] $\mathbf{E} \mathbf{L i}, \mathbf{Y} \mathbf{N i}$, Half-integral finite surgeries on knots in $S^{3}$, Ann. Fac. Sci. Toulouse Math. 24 (2015) 1157-1178 MR 
[14] W B R Lickorish, A representation of orientable combinatorial 3-manifolds, Ann. of Math. 76 (1962) 531-540 MR

[15] L Moser, Elementary surgery along a torus knot, Pacific J. Math. 38 (1971) 737-745 MR

[16] Y Ni, X Zhang, Finite Dehn surgeries on knots in $S^{3}$, Algebr. Geom. Topol. 18 (2018) 441-492 MR

[17] P Ozsváth, Z Szabó, Absolutely graded Floer homologies and intersection forms for four-manifolds with boundary, Adv. Math. 173 (2003) 179-261 MR

[18] P Ozsváth, Z Szabó, On the Floer homology of plumbed three-manifolds, Geom. Topol. 7 (2003) 185-224 MR

[19] P Ozsváth, Z Szabó, On knot Floer homology and lens space surgeries, Topology 44 (2005) 1281-1300 MR

[20] P Ozsváth, Z Szabó, On the Heegaard Floer homology of branched double-covers, Adv. Math. 194 (2005) 1-33 MR

[21] P Scott, The geometries of 3-manifolds, Bull. London Math. Soc. 15 (1983) 401-487 MR

[22] W P Thurston, Three-dimensional manifolds, Kleinian groups and hyperbolic geometry, Bull. Amer. Math. Soc. 6 (1982) 357-381 MR

[23] A H Wallace, Modifications and cobounding manifolds, Canadian J. Math. 12 (1960) 503-528 MR

Department of Mathematics, Princeton University

Princeton, NJ, United States

Department of Mathematics, California Institute of Technology

Pasadena, CA, United States

Department of Mathematics, Stanford University

Stanford, CA, United States

Department of Mathematics, California Institute of Technology

Pasadena, CA, United States

Department of Mathematics, University of Texas, Austin

Austin, TX, United States

Department of Mathematics, Duke University

Durham, NC, United States

wballinger@princeton.edu, chingyunhsu22@gmail.com, wmackey@stanford.edu, yini@caltech.edu, tochse@utexas.edu, vafaee@math.duke.edu

Received: 17 May 2018 Revised: 6 June 2019 
